\title{
Structural Characterization of Proteasome Inhibition
}

\author{
Dissertation \\ for the award of the degree \\ "Doctor rerum naturalium" (Dr. rer. nat.) \\ of the Georg-August-Universität Göttingen
}

within the doctoral program

Biomolecules: Structure - Function - Dynamics

of the Göttingen Graduate School for Neuroscience, Biophysics and

Molecular Biosciences (GGNB)

submitted by

Jil Schrader

from Geseke, Germany

Göttingen, 2017 

Thesis Committee

Prof. Dr. Holger Stark (Reviewer)

Structural Dynamics, Max Planck Institute for Biophysical Chemistry

Prof. Dr. Ralf Ficner (Reviewer)

Molecular Structural Biology, Göttingen Center for Molecular Biosciences

Dr. Karin Kühnel

until 2016: Neurobiology, Max Planck Institute for Biophysical Chemistry

\section{Members of the Examination Board}

Prof. Dr. Holger Stark

Structual Dynamics, Max Planck Institute for Biophysical Chemistry

Prof. Dr. Ralf Ficner

Molecular Structural Biology, Göttingen Center for Molecular Biosciences

Prof. Dr. Blanche Schwappach

Molecular Biology, University Medical Center Göttingen

Dr. Alexander Stein

Membrane Protein Biochemistry, Max Planck Institute for Biophysical Chemistry

Prof. Dr. Henning Urlaub

Bioanalytical Massspectrometry, Max Planck Institute for Biophysical Chemistry

Prof. Dr. Detlef Doenecke

Molecular Biology, University Medical Center Göttingen

Date of the oral examination: 22.05.2017 
I hereby declare that the $\mathrm{PhD}$ thesis 'Structural Characterization of Proteasome Inhibition' has been written independently with no other aids or sources than quoted. This thesis (wholly or in part) has not been submitted elsewhere for any academic award or qualification.

$$
7 \cdot \int d i d f
$$




\section{Contents}

List of Publications $\quad$ v

Abstract vi vi vis

1 Introduction 1

1.1 Ubiquitin-Mediated Proteolysis . . . . . . . . . . . . . . 1

1.1.1 The Ubiquitin-Proteasome Pathway Mechanism . . . . . . . . . . 1

1.2 The $20 \mathrm{~S}$ and $26 \mathrm{~S}$ Proteasome . . . . . . . . . . . . . 4

1.2.1 The 20S Proteasome - Structure and Function . . . . . . . . . . 4

1.2.2 The 20S Catalytic Mechanism - Proteolysis . . . . . . . . . . 7

1.2.3 The 26S Proteasome - Structure and Function . . . . . . . . . 8

1.3 Proteasome Inhibition . . . . . . . . . . . . . . . . 13

1.3.1 Boronic Acid Inhibitors . . . . . . . . . . . . . . . . . 15

1.3.2 Epoxyketone Inhibitors . . . . . . . . . . . . . . . . . 16

1.3.3 Ketoaldehyde Inhibitors . . . . . . . . . . . . . . . 16

1.3.4 Recent Advances in Proteasome Inhibitor Development . . . . . . 17

1.3.5 Biological Implication of Proteasome Inhibition . . . . . . . . . . 18

1.4 An Introduction to X-ray Crystallography and Single Particle Electron Cryomicroscopy ....................... 20

1.4.1 X-ray Crystallography . . . . . . . . . . . . . . . . 20

1.4.2 Single Particle Electron Cryomicroscopy . . . . . . . . . . . . 23

1.4.3 Advantages and Disadvantages .............. . . 24

1.5 Objectives . . . . . . . . . . . . . . . . . . . . 27

2 Materials and Methods $\quad 29$

2.1 Materials . . . . . . . . . . . . . . . . . . . 29

2.1.1 Software .......................... 29

2.1.2 Special Equipment . . . . . . . . . . . . . . 30

2.1.3 Chemicals ........................ 31

2.1.4 Buffers . . . . . . . . . . . . . . . . . 33

2.2 Methods . . . . . . . . . . . . . . . . . . 34

2.2.1 Protein Purification . . . . . . . . . . . . . . . 34

2.2.1.1 Purification of 20S Proteasomes from HeLa cells . . . . . 34

2.2.1.2 Purification of 26/30S Proteasomes from HeLa Cells . . 35 
2.2 .2 Enzyme Kinetics . . . . . . . . . . . . . . . . 36

2.2.3 Electronic Structure Calculations . . . . . . . . . . . . . . 37

2.2.4 Crystallization Methods ................ 38

2.2.4.1 Preparation of Protein Crystals . . . . . . . . . . 38

2.2.4.2 Postcrystallization Treatment . . . . . . . . . . . 39

2.2.5 X-ray Analysis Methods . . . . . . . . . . . . . . . . . . . 40

2.2.5.1 Crystal Mounting and Diffraction Data Collection . . . . 40

2.2.5.2 Structure Determination . . . . . . . . . . . 40

2.2.5.3 Identification of Ions . . . . . . . . . . . . . . . 40

2.2.6 Sample Preparation for Electron Microscopy . . . . . . . . . . . . 41

2.2.6.1 GraFix .................... . . . 41

2.2.6.2 Negative Staining . . . . . . . . . . . . . . . . . 41

2.2.6.3 Vitrification . . . . . . . . . . . . . 42

2.2.7 Imaging of Electron Microscopic Data . . . . . . . . . . . . . . 42

2.2.8 Image Processing . . . . . . . . . . . . . . . . . . . 42

2.2.8.1 Micrograph Analysis and Particle Picking . . . . . . . 42

2.2.8.2 2D Processing . . . . . . . . . . . . . . . 43

2.2.8.3 Initial Model Building . . . . . . . . . . . . . . . 43

2.2.8.4 Conformational Sorting . . . . . . . . . . . 43

2.2.8.5 Refinement and Resolution Determination . . . . . . . 43

2.2.8.6 Model Building . . . . . . . . . . . . . . . 44

2.2.8.7 Validation . . . . . . . . . . . . . . . . 44

2.2.9 Visualization of the Conformational Space of the Human 26S Pro-

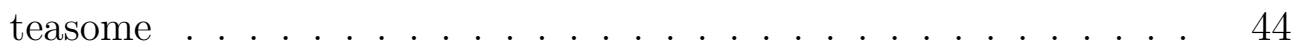

$\begin{array}{lll}3 & \text { Results } & 47\end{array}$

3.1 Native Human 20S Proteasomes and in Complex with Inhibitors . . . . . 47

3.1.1 Purification and Crystallization of the Human 20S Proteasome . . 47

3.1.1.1 Preparation of Human 20S Proteasome Crystals . . . . . 49

3.1.1.2 Postcrystallization Treatments . . . . . . . . . 49

3.1.2 Native Crystal Structure of the Human 20S Proteasome . . . . . . 51

3.1.3 Crystal Structures of Seven Inhibitor Complexes of the Human 20S Proteasome ......................... 53

3.1.4 Structural Implications of the Six-ring Linkage . . . . . . . . . . 58

3.1.5 Elucidation of the Inhibition Mechanism of Epoxyketone Inhibitors 60

3.2 Structural Impact of 20S Inhibitors on the Human Proteasome Holoenzyme 65

3.2.1 Purification and Analysis of the Human 26S Proteasome in Complex with Oprozomib . . . . . . . . . . . . . . . 65 
3.2.2 EM Structure of the Human 26S Proteasome in Complex with Oprozomib ........................ 68

3.2.3 Restricted Dynamics in the Inhibited 26S Proteasome . . . . . . . 72

3.2.4 The Energy Landscape of the Lid Movements . . . . . . . . . . . 74

4 Discussion $\quad 77$

4.1 Pipeline for Structure-based Analysis of Human Proteasome Inhibition . 78

4.2 Native Human 20S Proteasomes Structure at $1.8 \AA$ Resolution . . . . . . 80

4.3 Human 20S Proteasome Inhibition . . . . . . . . . . . . . 82

4.4 Structural Impact of $20 \mathrm{~S}$ Inhibitors on the $26 \mathrm{~S}$ Holoenzyme . . . . . . . . 88

4.5 A Potential bi-directional Signaling Pathway . . . . . . . . . . . . . . 91

$\begin{array}{lll}5 & \text { Conclusion and Outlook } & 97\end{array}$

Appendices

$\begin{array}{ll}\text { A Declaration of contribution as co-author } & \text { I }\end{array}$

B Abbreviations

Bibliography

$\begin{array}{ll}\text { Acknowledgements } & \text { XXIII }\end{array}$

$\begin{array}{ll}\text { Curriculum Vitae } & \text { XXV }\end{array}$ 


\section{List of Publications}

This thesis is based on the following publication and manuscript:

1. J. Schrader*, F. Henneberg*, R. A. Mata, K. Tittmann, T. R. Schneider, H. Stark, G. Bourenkov, A. Chari. (2016). The inhibition mechanism of human 20S proteasomes enables next-generation inhibitor design. Science. 353(6299), 594-598.

2. D. Haselbach*, J. Schrader*, F. Lambrecht, F. Henneberg, A. Chari, H. Stark. Long range allosteric regulation of the human $26 \mathrm{~S}$ proteasome by $20 \mathrm{~S}$ proteasometargeting cancer drugs. Manuscript in preparation.

* authors contribute equally to this work.

In addition, parts of this work are registered for patent:

1. H. Stark, A. Chari, J. Schrader, F. Henneberg. (2016). Method for the purification of biological macromolecular complexes. EP 16173037.9 and USSN 62/345,913. Filed January 2016. Patent Pending.

2. H. Stark, A. Chari, J. Schrader, F. Henneberg. (2016). Next-generation proteasome inhibitor design. EP 16173057.7 and USSN 62/346,203. Filed January 2016. Patent Pending. 



\section{Abstract}

The proteasome plays a major role in non-lysosomal protein degradation pathways and inhibition of proteasomes became a valid therapeutic strategy for anti-cancer therapy in the recent years. Current efforts focus on the identification and development of second generation inhibitors with enhanced pharmacological properties. For this, improved structural knowledge and an advanced biochemical characterization of proteasome inhibition is necessary.

In this work, I present a reproducible and robust pipeline that enables the purification, crystallization, crystal handling and structure determination of the native 20S proteasome and 20S-inhibitor complexes. Crystal structures including four previously uncharacterized inhibitor complexes with clinically relevant inhibitors were elucidated at resolutions between 1.8 and $2.1 \AA$. The improved resolutions allow to define the inhibition chemistry at atomic resolution. This led to revised descriptions of binding modes for epoxyketone and ketoaldehyde inhibitors, a redefinition of the proteasome active site, and a concept for future drug design.

Single particle electron cryomicroscopy (cryo-EM) was the method of choice when studying the influence of $20 \mathrm{~S}$ core particle inhibition on the dynamic $26 \mathrm{~S}$ holoenzyme. We determined structures of the $26 \mathrm{~S}$ proteasome with and without inhibitor and report for the first time structural changes of $26 \mathrm{~S}$ proteasomes upon inhibition. Drug binding limits the conformational space of the $19 \mathrm{~S}$ regulatory particle and we identified an energy barrier that stabilizes the proteasome in a non-productive state. Here, we describe that core particle inhibition triggers a structural long-range allosteric regulation of the human $26 \mathrm{~S}$ proteasome.

This thesis offers insights into the exact catalytic mechanism of peptide cleavage and proteasome inhibition in the human $20 \mathrm{~S}$ proteasome and a detailed description of the proteolytic site. In addition, the structural impact of $20 \mathrm{~S}$ inhibition on the human $26 \mathrm{~S}$ proteasome was studied for the first time and the findings allow for a new way to screen and develop future allosteric proteasome inhibitors. A reproducible workflow to structurally study inhibitor-proteasome complexes by advanced protein complex purification combined with X-ray crystallography or single particle cryo-EM is presented.

Keywords: protein complex purification, X-ray crystallography, electron cryomicroscopy, single particle image processing, structural dynamics, cancer therapeutics, drug design 


\section{Chapter 1}

\section{Introduction}

\subsection{Ubiquitin-Mediated Proteolysis}

Protein degradation is a major part of protein homeostasis in the cell. The concept of protein degradation is at the center of understanding the underlying causes of the evergrowing list of diseases associated with protein misfolding ${ }^{1}$ as well as aggregation-related degenerative disorders ${ }^{2}$. Proteins in the cell have half-lives that range from a few minutes to several days and the level of all proteins must be evaluated continuously to uphold the required balance. The cell's ability to adapt to changing conditions or to progress through its general life cycle, ultimately requires a dynamic composition of its proteome. To maintain normal cell function new proteins are synthesized and old or unused proteins are degraded ${ }^{3}$.

In the 1970s, an intracellular, non-lysosomal protein degradation pathway was discovered and today it is known that protein degradation in eukaryotes is almost entirely undertaken by a complex molecular machine, the proteasome ${ }^{4}$. The proteasome forms the center of the ubiquitin-proteasome system (UPS), with critical functions in cell cycle control, protein quality control, programmed cell death by apoptosis, inflammation, transcription and many more biological processes ${ }^{3}$. The extent of the UPS is facilitated by a broad range of ubiquitin ligase enzymes and specific factors. The proteasome is the only ATP-dependent protease found in eukaryotes and the only one that is ubiquitin dependent ${ }^{5}$. Structurally, the proteasome is a hybrid of a proteolytic and ATP-dependent regulatory machinery composed of a 20S core particle and 19S regulatory particle, respectively ${ }^{6}$.

\subsubsection{The Ubiquitin-Proteasome Pathway Mechanism}

Degradation of proteins by the UPS is energy dependent and includes two discrete and successive parts: (1) substrate recognition, whereby the substrate specificity is ensured by polyubiquitination of substrates by specific enzymes and (2) substrate unfolding to 
make the polypeptide backbone fully accessible for proteolytic cleavage ${ }^{7}$.

Proteins destined for degradation are modified with polymers of the highly conserved protein, ubiquitin. The covalent attachment of ubiquitin to the substrate is carried out by a series of enzymes (Figure 1.1):

Ubiquitin is first activated upon ATP turnover by an E1 ubiquitin activating enzyme, forming a high energy thiol ester with the carboxyl group at the C-terminus, thereby activating the C-terminus of ubiquitin for nucleophilic attack. Subsequently, the activated ubiquitin molecule is transfered to the active site cysteine of a second enzyme, the E2 ubiquitin conjugating enzyme. The ubiquitin ligase E3 recognizes its substrate, transfers the activated ubiquitin from the E2 enzyme to the substrate and catalyzes the formation of an isopeptide bond between a lysine residue of the substrate and the C-terminus of the ubiquitin. To form polyubiquitin chains, additional ubiquitin molecules are ligated to Lys48 of the ubiquitin that is already conjugated to the protein substrate ${ }^{8}$. Generally, a chain of four or more ubiquitin molecules is necessary to create a targeting signal for degradation by the proteasome and this three-step mechanism initiates all known ubiquitination reactions, independent of the biological fate ${ }^{9}$.

In human, numerous enzymes are involved in the UPS: two E1 enzymes, approximately 30 E2 conjugating enzymes and more than 500 E3 ubiquitin ligases, whereby the substrate selection is carried out by the different types of E3 ubiquitin ligases ${ }^{10}$.

Furthermore, deubiquitinating enzymes (DUBs) are part of the ubiquitin-proteasome pathway mechanism. These enzymes are responsible for removing ubiquitin from targeted proteins and disassemble polyubiquitin chains ${ }^{11}$. DUBs add an additional regulatory control step prior to protein degradation and are responsible for maintaining a free pool of mono-ubiquitin for protein degradation ${ }^{12}$. 


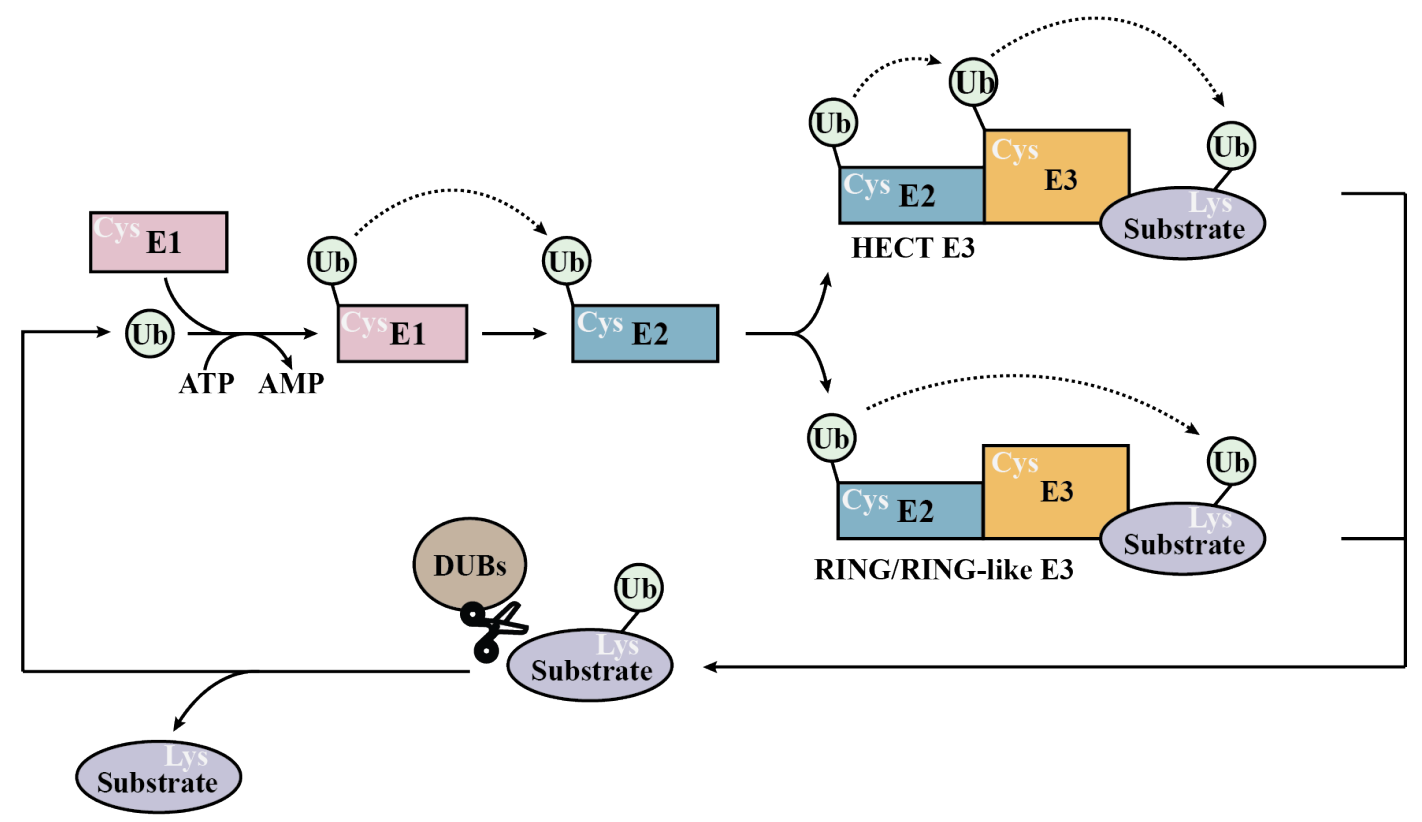

Figure 1.1: Overview of the ubiquitin conjugating mechanism. Ubiquitin molecules (Ub, green) are attached to substrate proteins (purple), which are destined for degradation. Ubiquitination results in the formation of a bond between the C-terminus of ubiquitin with the $\varepsilon$-amino group of a lysine of the substrate. In the UPS, a well-defined series of enzymes take part in the (poly-)ubiquitination reaction. Ubiquitin is first activated by the E1 enzyme (pink) under ATP hydrolysis and subsequently transferred to the E2 conjugating enzyme (turquoise). Next, the E3 ubiquitin ligase (orange) determines the transfer of ubiquitin to the substrate protein. Here, the transfer varies between the two major classes of E3 ubiquitin ligases, termed HECT and RING-like ${ }^{13,14}$. The ubiquitin-tagged substrate is then targeted by the $26 \mathrm{~S}$ proteasome for degradation (not shown). Ubiquitination is reversed by deubiquitinating enzymes (DUBs, brown). DUBs remove ubiquitin from proteins and disassemble polyubiquitin chains. Modified from Ravid and Hochstrasser ${ }^{15}$. 


\subsection{The 20S and 26S Proteasome}

\subsubsection{The 20S Proteasome - Structure and Function}

The $20 \mathrm{~S}$ proteasome is a large macromolecular complex of approximately $750 \mathrm{kDa}$, comprising 28 subunits arranged in four co-axially stacked heteroheptameric rings (Figure 1.2). The inner rings are formed by seven distinct $\beta$-subunits, whereas the outer rings consist of seven different $\alpha$-subunits. Three $\beta$-subunits contain proteolytic active sites $(\beta 1, \beta 2, \beta 5)$, belonging to the N-terminal nucleophile hydrolase family. These subunits are located deep within the barrel-shaped structure of the core particle, likely to avoid any unspecific degradation events and also to maintain a controlled, sequential degradation of substrates ${ }^{16}$.

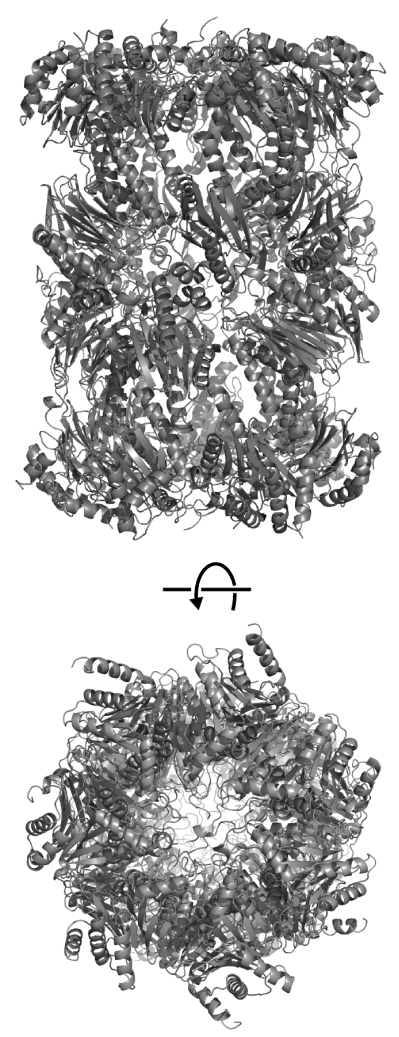

Top-view: closed gate

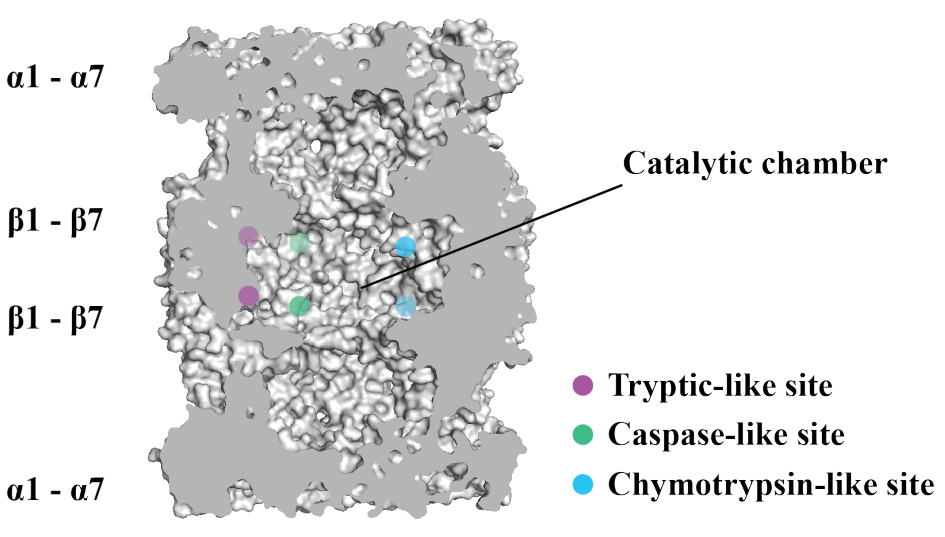

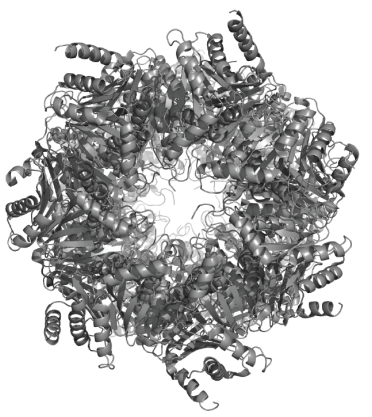

Top-view: opened gate

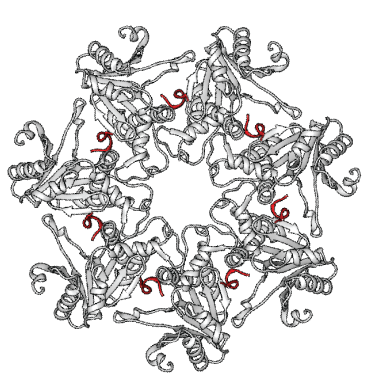

$\alpha$-ring with

HbYX motifs

Figure 1.2: Structural details of the 20S proteasome. Top: Side view and cross section of a spacefilling representation of the yeast $20 \mathrm{~S}$ proteasome (PDB ID: 1RYP) ${ }^{16}$. Purple dots denote the spatial position of the $\beta 2$ (tryptic-like), green dots the $\beta 1$ (caspase-like), and blue dots the $\beta 5$ (chymotrypticlike) active site, respectively. Bottom: Top view on the 20S proteasome. Bottom left: Wild-type yeast 20S proteasome (PDB ID: 1RYP), representing a closed gate. Bottom middle: Opened-gate mutant of the yeast $20 \mathrm{~S}$ proteasome (PDB ID: $1 \mathrm{G} 0 \mathrm{U})^{17}$. Bottom right: View onto the $\alpha$-ring of the $20 \mathrm{~S}$ particle form T. acidophilum, in complex with the PAN C-terminus. The HbYX-motifs (red) which are inserted into the pockets formed at the interfaces of two adjacent $\alpha$-subunits are shown. The HbYX motifs bind to a lysine residue in the $\alpha$-pocket. Modified from Kriegenburg et al. ${ }^{18}$ 
Each active site cleaves preferentially after particular amino acid residues: (1) The $\beta 1$ subunit has been classified as a caspase-like active site, because cleavage occurs after acidic residues, (2) $\beta 2$ harbors a tryptic-like activity, cleaving after basic residues, and (3) $\beta 5$ is termed chymotryptic-like, because cleavage occurs preferentially after bulky hydrophobic residues ${ }^{19}$. Studies have determined that the 20 S proteasome shows preference for certain peptide bonds over others for cleavage. The active sites are much less specific with regard to the recognition of certain amino acid residues in $\mathrm{P} 1$ position than classification based upon fluorogenic peptide substrates would suggest ${ }^{20}$. According to a model by Schechter and Berger ${ }^{21}$, amino acid residues in a substrate undergoing cleavage are designated P1, P2, P3, P4 etc. in the N-terminal direction from the cleaved bond.

The 20S proteasome has a narrow channel in the center of the outer $\alpha$-rings that only allows the passage of unfolded polypeptides, which then access the inner chamber and proteolytic active sites. The $\mathrm{N}$ - terminal tails of the $\alpha$-subunits form a gate, its closed or open states prevent or allow the passage of substrates through the channel, respectively (Figure 1.2) ${ }^{17}$. To function in vivo, the $20 \mathrm{~S}$ proteasome needs to associate with regulatory particles that partially determine the specificity of protease function.

The $20 \mathrm{~S}$ proteasome is highly conserved from archaea to higher eukaryotes. The striking features of this protease were structurally analyzed by X-ray crystallography and electron microscopy. Atomic structures of the $20 \mathrm{~S}$ proteasome from yeast and mammals have been determined and their overall structures and subunit arrangements are nearly identical $^{16,22,23}$.

X-ray crystallography is a method that enables to gain three dimensional structures of biological molecules at resolutions below $1 \AA$. The resolution of a structure measured in Ångström $(\AA)$ is an important indicator towards its quality and reliability. Resolution in general describes the distance of two points which can be significantly discerned from each other. To determine a structure's resolution, one can directly investigate the structural features (Figure 1.3): Structures around $20 \AA$ show the overall shape. Molecular details are difficult to interpret in structures between 20 and $10 \AA$, though proteins and domains can be assigned. Between 9 and $6 \AA$ alpha helices become visible. Details begin emerging around $5 \AA$ when secondary structure elements can be determined; $\beta$-sheets become separated and bulky side chains become visible. Starting at $4 \AA$ more side chains appear in the density. Nucleotide bases start being separated and most side chains are visible at a resolution of $3.5 \AA$. In crystal structures resolved to $2 \AA$, most of the rotamers appear in their correct conformation and at $1.6 \AA$ the electron density is well enough defined to see holes in aromatic amino acids. From $1.1 \AA$ resolution, the electron density allows positions of hydrogen atoms to be resolved.

The first crystal structure of the 20S proteasome was published in 1996 and revealed details of the Thermoplasma acidophilum 20S proteasome at a resolution of $3.4 \AA^{24}$. In the 


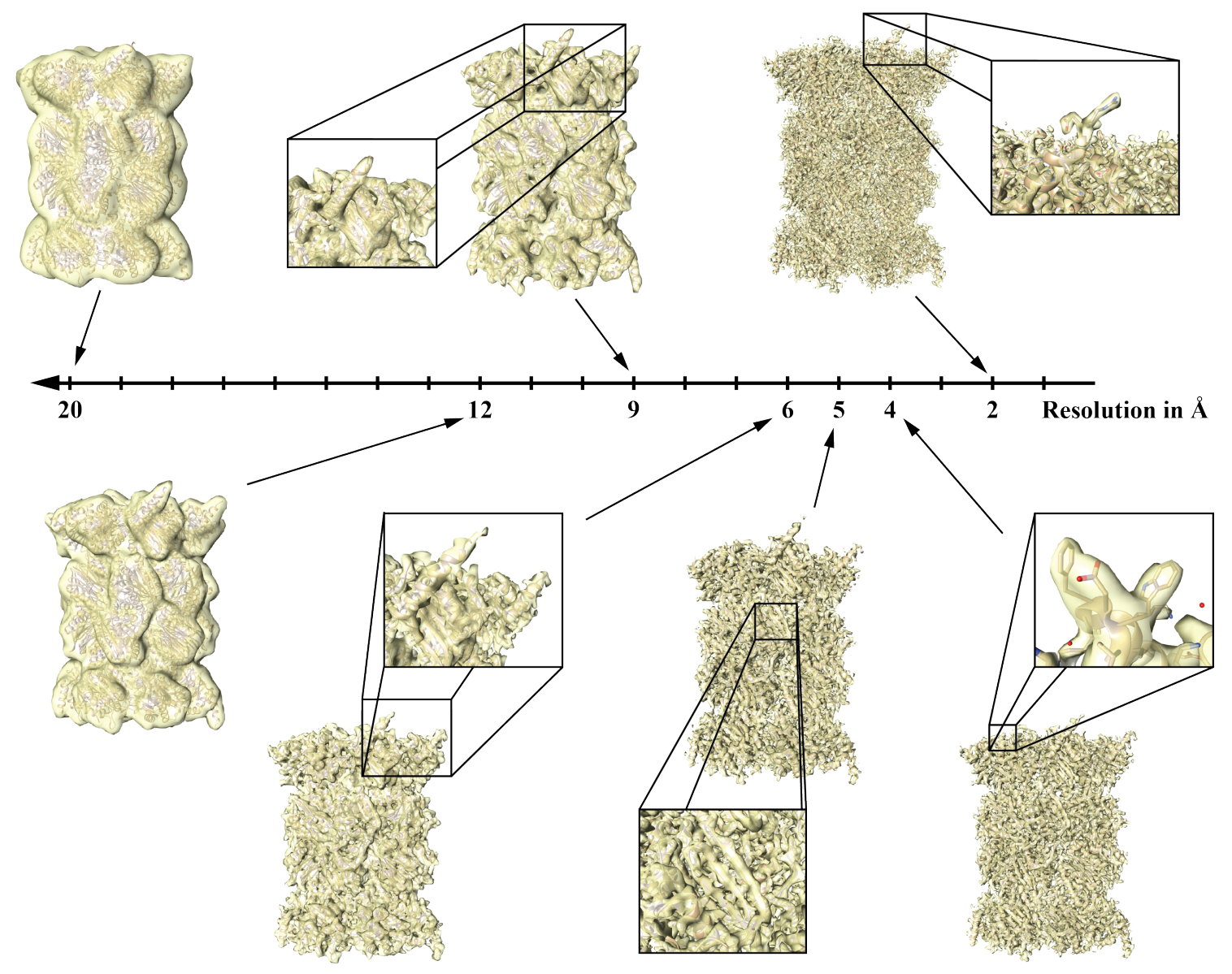

Figure 1.3: Structural details at distinct resolutions. The atomic model of the 20S proteasome is filtered to different resolutions. The depicted resolutions are defined by features which become visible in the structure. At $20 \AA$, the overall shape of the $20 \mathrm{~S}$ proteasome is recognizable. Individual domains become visible at $12 \AA$ and at $9 \AA$ the $\alpha$-helices are structurally defined. Between 6 and $5 \AA \beta$-sheets get separated. Starting at $4 \AA$, side chains become visible increasingly until at $2 \AA$ all side chains are visible.

following years, $20 \mathrm{~S}$ proteasomes from yeast $(S \text {. cerevisiae })^{16}$ and mammals (Bos taurus ${ }^{23}$ and Mus musculus ${ }^{22}$ ) were presented at resolutions between 2.4 and $3.2 \AA$, as well as the human $20 \mathrm{~S}$ proteasome structure at $2.6 \AA$ resolution ${ }^{25}$. Superposition of the individual catalytic $\beta$-subunits revealed that the structures from different organisms adopt very similar folds. Further, the superpositions of the $\alpha$-ring of the human proteasome with the $\alpha$-ring of the proteasomes from $S$. cerevisiae, Bos taurus and Mus musculus illustrates high structural conservation ${ }^{25}$.

In the crystal structures of free $20 \mathrm{~S}$ proteasomes from higher organisms, the entrance to the internal proteolytic chamber containing catalytic sites is usually closed by the Nterminal tails of the $\alpha$-subunits ${ }^{16}$. In yeast, the N-termini of the $\alpha 1, \alpha 2, \alpha 3, \alpha 6$, and $\alpha 7$ fill the opening in several layers (Figure 1.2). It is assumed that this gate must be opened for substrate processing in fully activated proteasomes ${ }^{17}$. Only substantial rearrangements would allow access of peptide substrates into the catalytic chamber ${ }^{17}$. 


\subsubsection{The 20S Catalytic Mechanism - Proteolysis}

Despite the fact that the catalytic sites of the 20S proteasome are unique in their chemical nature of substrate-binding and substrate preference, all sites employ an identical reaction mechanism for hydrolysis of the substrates peptide bonds. All three $\beta$-type subunits contain catalytically active threonine residues at their N-termini and employ a N-terminal nucleophile (Ntn) hydrolase activity.

The catalytic system is formed by the N-terminal threonine (Thr1), providing both the catalytic nucleophile and the primary proton acceptor, together with Glu17 and Lys33, first described in T.acidophilum ${ }^{24,26}$. With structural and mutational studies it became clear that the residues Ser129, Ser169 and Asp199 in close proximity to Thr1 are required for catalysis ${ }^{26,24}$. Additionally, Groll et al. proposed a fully occupied solvent molecule (NUK) in all three catalytic sites of the yeast 20S proteasomal complex. This molecule might be located close to the Thr1 $\mathrm{O} \gamma$ and N, Ser129 $\mathrm{O} \gamma$ and N and Gly47 $\mathrm{N}^{16}$.

With this knowledge a mechanism for proteolysis was proposed by Groll et al.:

During proteolysis, a nucleophilic attack of the hydroxyl group of Thr1 on the carbonyl carbon atom of the peptide bond takes place and the first cleavage product and a covalent acyl-enzyme intermediate are created. Next, the NUK water molecule, localized in close proximity to the Thr 1 acts as a proton shuttle to regenerate the enzyme and the peptide fragment is released ${ }^{16}$. The mechanism of substrate proteolysis is depicted as a schematic representation in Figure 1.4.

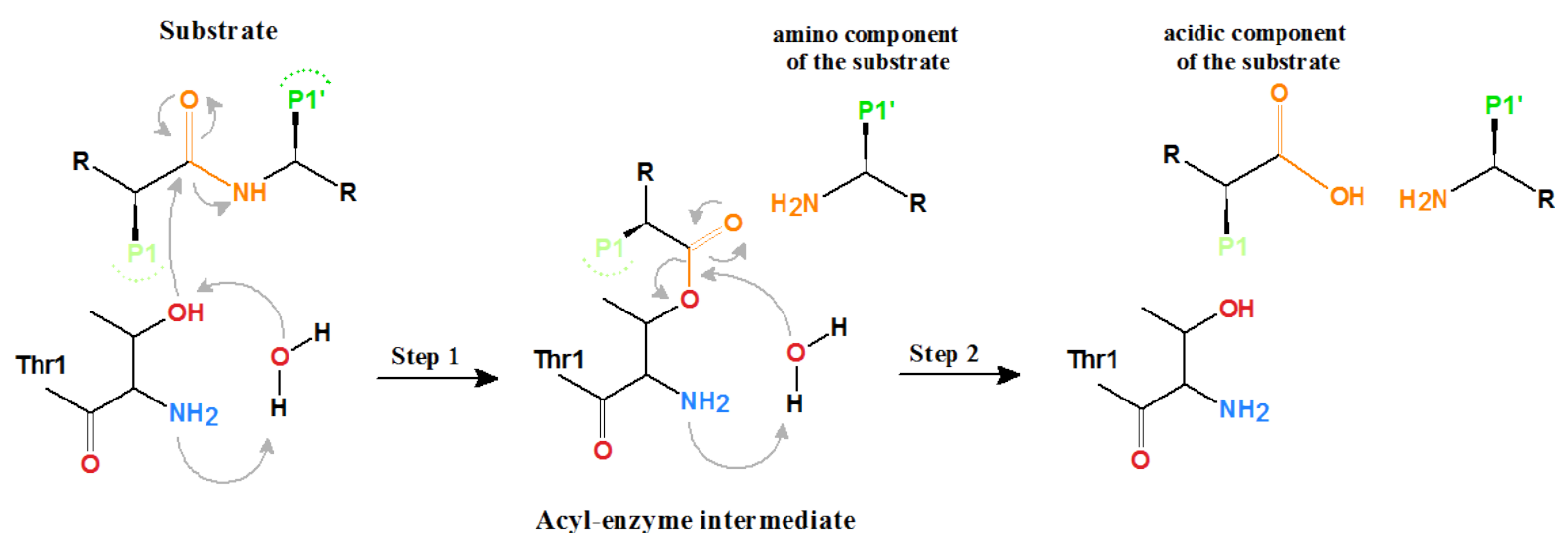

Figure 1.4: Substrate proteolysis. Schematic representation of the proteolytic cleavage mechanisms of the 20S proteasome. The substrates peptide bond is hydrolyzed by the N-terminal Threonine of the proteolytic active $\beta$ subunit. 


\subsubsection{The 26S Proteasome - Structure and Function}

The 2.5 MDa 26S proteasome is a large and complex member of the superfamily of ATP-dependent "chambered" proteases found in all domains of life ${ }^{27,7}$. The human $26 \mathrm{~S}$ proteasome is a labile complex that can dissociate into the $20 \mathrm{~S}$ core particle $(\mathrm{CP})$ and the $19 \mathrm{~S}$ regulatory particle (RP). The $20 \mathrm{~S}$ particle exhibits the proteolytic activity and the $19 \mathrm{~S}$ regulatory particle is responsible for substrate recognition, deubiquitination, unfolding and translocation of substrates into the core particle. The regulatory particle consists of at least 19 subunits, whereby the particle can split into two distinct assemblies: the lid and the base (Figure 1.5).

The base consists of nine subunits: Rpt1-6 (regulatory particle triple A protein 1-6) are paralogous AAA + ATPases and Rpn1, 2, 10 and 13 (regulatory particle non-ATPase) are non-ATPases. The six RP triphosphatases form a heterohexameric ATPase ring with the arrangement Rpt1-Rpt2-Rpt6-Rpt3-Rpt4-Rpt5 ${ }^{28}$, typically converting ATP hydrolysis into mechanical force and being in direct contact with the $\alpha$-ring of the core particle. Rpn1 and 2 are the largest subunits of the proteasome, exhibiting $\alpha$-helical proteasome/cyclosome repeats $(\mathrm{PC})$, which function as a docking platform for extrinsic ubiquitin receptors and deubiquitinating enzymes (DUBs). Rpn13 and Rpn10 directly recognize and bind ubiquitin, acting both as intrinsic receptors for polyubiquitinated substrates. With a position in the periphery of the proteasome, the ubiquitin receptors Rpn10 and Rpn13 sit in a perfect orientation to take part in the first steps of substrate recognition (Figure 1.5).

The RP lid consists of nine different Rpn subunits: Rpn3, 5-9, 11, 12 and Rpn15, whereby six of them are Proteasome/CSN/Initiation complex (PCI) domains (Rpn3, 5, 6, 7, 9, and 12). The PCI domain is a purely $\alpha$-helical domain of approximately 200 amino acid residues, which is generally localized at the extreme C-terminus of the protein. Rpn8 and Rpn11 are two MPN subunits, forming a heterodimer. The MPN domain spans approximately 140 amino acid residues present at the N-terminus of the proteins. Both the PCI and MPN domains might serve as structural scaffolds to uphold the proteasome's complex structure ${ }^{29}$. Rpn15 (DSS1 in human) is a small acidic domain involved in ubiquitin-substrate binding ${ }^{30}$. Rpn11 belongs to the DUBs. Structurally, the Rpn11 subunit sits above the central pore of the AAA + ATPase with its catalytic MPN domain $^{31}$. Due to its central position, Rpn 11 might be the primary DUB in proteasome degradation and is able to cleave off the entire ubiquitin chain from substrates which are directed from the 19S regulatory particle to the central pore of the $20 \mathrm{~S}$ core particle (Figure 1.5). Mutations of Rpn11 in yeast show that the activity of the domain is linked to severe growth defects ${ }^{32}$.

In addition to the $19 \mathrm{~S}$ proteasomal subunits, a number of proteins associate with the proteasome via loose, salt-labile interactions. These proteins play important roles in reg- 
ulating the proteolytic function ${ }^{33}$. Some serve as alternative ubiquitin receptors, such as the UBL/UBA domain containing proteins Rad23 (radiation sensitive 23), Dsk2 (dominant suppressor of Kar2) and Ddi1 (DNA damage-inducible 1), which help to deliver specific ubiquitin conjugates to the proteasome ${ }^{34}$. For example, proteins which trim or extend the ubiquitin chains of substrates on the regulatory particle are the deubiquitin enzyme Ubp6/USP14 (ubiquitin-specific protease 6 in yeast and 14 in mammals) and the ubiquitin ligase Hul5 (HECT ubiquitin ligase 5 in yeast) and its mammalian ortholog, KIAA $10^{35,36}$.

Within the 26S proteasome one of the contacts between 20S and 19S particles occurs through the Rpn5 and Rpn6 lid domains, with their N-termini extended towards the $\alpha 1$ and $\alpha 2$ subunits of the 20S particle ${ }^{31}$. This allows a direct interaction between the lid and the core particle. The main contact between 19S and 20S particles was found between the $\alpha$-subunits of the 20S and the conserved HbYX (hydrophobic, tyrosine, any amino acid) motifs at the flexible C-termini of the ATPase domains ${ }^{37}$. The HbYX regions can dock at the $\alpha 3 / \alpha 4, \alpha 1 / \alpha 2$, and $\alpha 5 / \alpha 6$ pockets between alpha subunits, shown by crosslinking experiments $^{38}$. The details of these interactions are not conclusively clarified, but it is thought that binding of all three HbYX motifs to the $\alpha$-ring will cause it to undergo a conformational change that better allows for substrate entry into the proteolytic chamber $^{16,17}$. In Figure 1.2 the $\alpha$-ring of the $20 \mathrm{~S}$ particle form T. acidophilum together with the inserted HbYX motifs from the PAN C-terminus are shown.

Besides the ATP-dependent activator 19S there are a number of different types of "caps" or activators of the $20 \mathrm{~S}$ particle. The $11 \mathrm{~S}$ cap (proteasome activator 28/PA28/REG) and the bleomycin-sensitive 10 cap (Blm10/PA200), which stimulate the degradation of substrates without ATP hydrolysis and ubiquitin detection ${ }^{39}$. The AAA + ATPase p97 (CDC48/VCP) might also serve as an alternative cap, first shown for proteasomes from T. acidophilum and later for mammalian proteasomes ${ }^{40,41}$. Complexes of the $20 \mathrm{~S}$ core particle with one 19S regulatory particle on one end and another activator such as $11 \mathrm{~S}$ or Blm10 on the other end are reported and referred to as hybrid proteasomes.

In general, the $26 \mathrm{~S}$ proteasome is a dynamic molecular machine. As a consequence, this multiprotein complex is biochemically labile or unstable, especially when isolated from the cell. It is therefore challenging to work with these proteases, either for biochemical assays or structural studies. Still, during recent years, a series of cryo-EM reconstructions of the $26 \mathrm{~S}$ holoenzyme as well as of the isolated RP or lid were published and provide a much clearer picture of its architecture.

The overall shape of the molecule was observed long ago, but only recently published structures give insights into the structural arrangements of the subunits within the complex. In 2010, the first subnanometer-resolution structure of the complete $26 \mathrm{~S}$ proteasome was presented and individual subunits could be placed into the model ${ }^{42}$. Only over the 

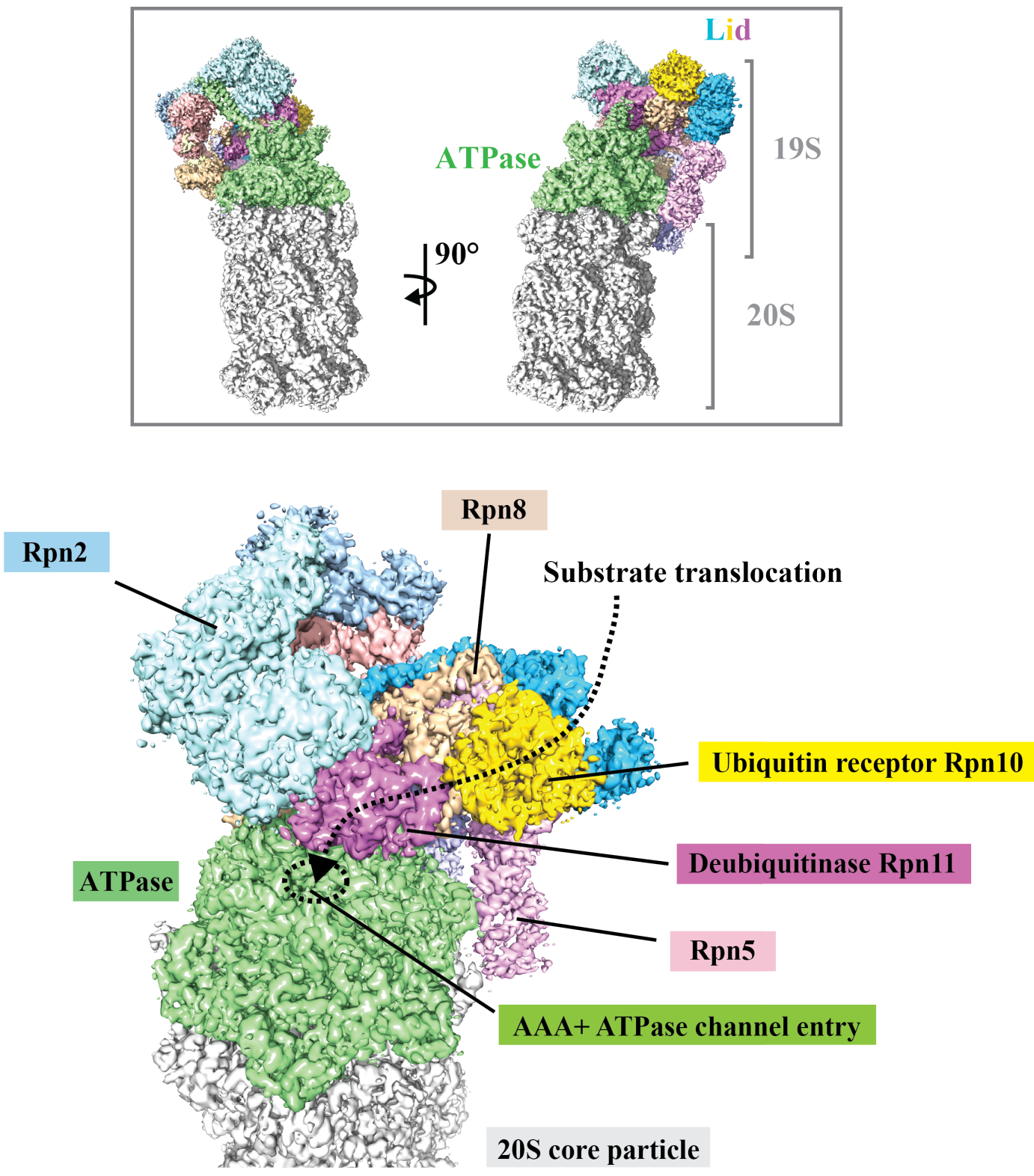

Figure 1.5: Structure of the 26S proteasome. Upper box: Architecture of the 26S proteasome, consisting of the $20 \mathrm{~S}$ core particle, ATPase and Lid-complex. Bottom: Half-top view onto the Lid/ATPase. Dashed arrow gives the direction of substrate translocation: Substrate is detected by one of the ubiquitin receptors (Rpn10, yellow) of the lid complex, ubiquitin is recycled by the deubiquitinating lid enzyme Rpn11 (purple) and enters the 20S core particle by a translocation through the pore of the AAA+ ATPase.

past 4 years several publications revealed the structure of the yeast and human 19S particle and the $26 \mathrm{~S}$ holoenzyme at higher resolutions ${ }^{31,43,42,44,45}$ with recently published structures of the human $26 \mathrm{~S}$ proteasome at $3.9,3.5$ and $4.4 \AA$ resolution, respectively ${ }^{46,47,48}$. An unexpected feature regarding the core particle and regulatory particle interaction was found in electron microscopic data of D. melanogaster and S. pombe 26/30S proteasomes: The pore of the heteroheptameric AAA + ATPase ring of the regulatory particle does not align with the pore of the $20 \mathrm{~S}$ core particle. It is shifted axially by almost $30 \AA$ and tilted vertically by $5-10^{\circ 49}$. The functional significance of the disalignment was not known, but 
recent structural data from the yeast proteasome offers an explanation for this offset. Three major conformational states of the proteasome holoenzyme have been found by cryo-EM analysis: S1, S2 and S3 ${ }^{50}$. These states form the basis for a hypothesis of ubiquitin/substrate binding, deubiquitination, substrate unfolding and translocation by the proteasome. S1 is defined as the ground state, which is the predominant state under ATP hydrolysis and without substrate present in the condition. In contrast, the S3 state is present upon excess of substrates ${ }^{51}$ or by introducing the non-hydrolyzable ATP analogue $\operatorname{ATP}_{\gamma} \mathrm{S}^{50}$ to the system. S2 is a hybrid state between $\mathrm{S} 1$ and $\mathrm{S} 3$. It is proposed that docking of substrates occurs in the S1 state, considered the substrate-accepting state, followed by a structural rearrangement when the substrate is accepted for degradation in S2 state. Finally, the substrate is unfolded, ubiquitin is removed and the substrate is translocated into the $20 \mathrm{~S}$ core particle for degradation in conformational state S3, the substrate-processing state. The main conformational rearrangements are a rotation of the lid by $25^{\circ}$ and a translation and rotation of the AAA + ATPase in relation to the $20 \mathrm{~S}$ core particle, resulting in an alignment of the ATPase ring and the core particle channel in S3. 
Table 1.1: 26S proteasome subunits. Listed are all subunits belonging to the core and regulatory particle of the $26 \mathrm{~S}$ proteasome.

\begin{tabular}{|c|c|c|c|}
\hline $\begin{array}{l}\text { Subunit } \\
\text { Name }\end{array}$ & Subcomplex & Function & Activity/domain \\
\hline$\alpha 1$ & 20S core particle & Scaffolding/gating & \\
\hline$\alpha 2$ & 20S core particle & Scaffolding/gating & \\
\hline$\alpha 3$ & 20S core particle & Scaffolding/gating & \\
\hline$\alpha 4$ & 20S core particle & Scaffolding/gating & \\
\hline$\alpha 5$ & 20S core particle & Scaffolding/gating & \\
\hline$\alpha 6$ & 20S core particle & Scaffolding/gating & \\
\hline$\alpha 7$ & 20S core particle & Scaffolding/gating & \\
\hline$\beta 1$ & 20S core particle & Protease & Caspase-like \\
\hline$\beta 2$ & 20S core particle & Protease & Tryptic-like \\
\hline$\beta 3$ & 20S core particle & Scaffold & \\
\hline$\beta 4$ & 20S core particle & Scaffold & \\
\hline$\beta 5$ & 20S core particle & Protease & Chymotryptic-like \\
\hline$\beta 6$ & $20 \mathrm{~S}$ core particle & Scaffold & \\
\hline \multirow[t]{2}{*}{$\beta 7$} & 20S core particle & Scaffold & \\
\hline & Base & & \\
\hline Rpt1 & $19 \mathrm{~S}$ regulatory particle & Unfoldase & AAA+ ATPase \\
\hline Rpt2 & $19 \mathrm{~S}$ regulatory particle & Unfoldase & $\mathrm{AAA}+$ ATPase \\
\hline Rpt3 & $19 \mathrm{~S}$ regulatory particle & Unfoldase & $\mathrm{AAA}+$ ATPase \\
\hline Rpt4 & $19 \mathrm{~S}$ regulatory particle & Unfoldase & $\mathrm{AAA}+$ ATPase \\
\hline Rpt5 & $19 \mathrm{~S}$ regulatory particle & Unfoldase & $\mathrm{AAA}+$ ATPase \\
\hline Rpt6 & $19 \mathrm{~S}$ regulatory particle & Unfoldase & $\mathrm{AAA}+$ ATPase \\
\hline Rpn1 & $19 \mathrm{~S}$ regulatory particle & Substrate recruitment & $\mathrm{PC}$ repeats \\
\hline Rpn2 & $19 \mathrm{~S}$ regulatory particle & Rpn13 docking & PC repeats \\
\hline Rpn10 & $19 \mathrm{~S}$ regulatory particle & Ubiquitin receptor & vWA/UIM domain \\
\hline \multirow[t]{2}{*}{ Rpn13 } & $19 \mathrm{~S}$ regulatory particle & Ubiquitin receptor & PRU domain \\
\hline & Lid & & \\
\hline Rpn3 & $19 \mathrm{~S}$ regulatory particle & Ubiquitin receptor & PCI domain \\
\hline Rpn5 & $19 \mathrm{~S}$ regulatory particle & Ubiquitin receptor & PCI domain \\
\hline Rpn6 & $19 \mathrm{~S}$ regulatory particle & Ubiquitin receptor & PCI domain \\
\hline Rpn7 & $19 \mathrm{~S}$ regulatory particle & Ubiquitin receptor & PCI domain \\
\hline Rpn8 & $19 \mathrm{~S}$ regulatory particle & Ubiquitin receptor & $\mathrm{JAMM} / \mathrm{MPN}+$ domain \\
\hline Rpn9 & $19 \mathrm{~S}$ regulatory particle & Ubiquitin receptor & PCI domain \\
\hline Rpn11 & $19 \mathrm{~S}$ regulatory particle & Deubiquitinylation & JAMM/MPN+ domain \\
\hline Rpn12 & $19 \mathrm{~S}$ regulatory particle & Scaffold & PCI domain \\
\hline Rpn15 & $19 \mathrm{~S}$ regulatory particle & Ubiquitin receptor & IDP \\
\hline
\end{tabular}




\subsection{Proteasome Inhibition}

The proteasome is a key molecular complex for the degradation of proteins that control the cell division cycle and apoptosis. It is therefore an interesting target for therapeutic agents that inhibit cell proliferation in diseases such as cancer. Proteasome inhibition is an accepted antineoplastic strategy since the approval of Bortezomib in $2003^{52}$. Within nine years, Bortezomib was raised from development to its first approval by the US Food and Drug Administration (FDA) for the treatment of multiple myeloma, with an annual sales value of two billion US Dollars. The success of Bortezomib has drawn further interest in proteasomes as targets in oncology, and today at least five other compounds are at various stages of clinical approval for treatment of multiple myeloma, a cancer of plasma cells ${ }^{53,54}$.

Inhibition of proteasomes leads to the accumulation of misfolded proteins and the formation of toxic, reactive oxygen species. At the same time it induces apoptosis in cancerous cells, which lack the ability to enter cell cycle arrest ${ }^{55}$. In preclinical studies, researchers studied the effectiveness of proteasome inhibitors on different types of cancers and found a selectivity for malignant cells ${ }^{56,57}$. It was found that actively proliferating malignant cells are more sensitive to proteasomal inhibition than non-cancerous cells ${ }^{58,59}$. The underlying mechanisms of the increased susceptibility of malignant cells are not yet completely understood. Malignant cells have characteristics that might play a role in the biological basis for this cytotoxic mechanism: malignant cells proliferate rapidly and have therefore an increased demand for proteasomal degradation. This hypothesis is consistent with findings in studies of lymphocytes. Chronic Lymphocytic Leukemia (CLL) lymphocytes have a three-fold higher activity of the chymotryptic-like active site and an upregulated ubiquitin-proteasome system with higher levels of ubiquitin-conjugated proteins compared to healthy lymphocytes ${ }^{60}$. Inhibition of the proteasome in cancerous lymphocytes would result in an accumulation of proteins and terminate in apoptosis.

The first clinically approved drug Bortezomib revealed substantial off-target activity by reacting with various enzymes that results in severe side effects ${ }^{61}$. Furthermore, resistance to this drug remains a clinically significant problem ${ }^{62}$. In recent years, second-generation competitive inhibitors have been discovered and many are under evaluation by the FDA. The design principle for these inhibitors involves a combination of a peptide moiety coupled with a reactive head group acting as electrophilic anchor, such as for example $\alpha$ ', $\beta$ '-epoxyketones, boronates, aldehydes, $\alpha$-ketoaldehydes, vinyl sulfones or $\beta$-lactones. Most of the recently published structural knowledge about proteasome inhibition including the mechanism of inhibitor binding and the binding-site composition derives from studies of the yeast 20S proteasome. Only little is known about the inhibitory mechanisms in the human system, whereas it is assumed that inhibition takes place in a similar 
manner. The first structures of the human 20S proteasome in complex with inhibitors were only recently determined by X-ray crystallography and electron microscopy at resolutions of $2.9 \AA$ and $3.5 \AA$, respectively ${ }^{25,63}$. Due to the relatively low resolution of these structures, direct observations of atomic details, especially at the binding site of inhibitors, was not possible. Nevertheless, predicted models for the inhibition mechanism of various inhibitor classes were published and are presented in the following sections.

Structures of different 20S proteasome-inhibitor complexes were elucidated and analyzed for this thesis. Numerous inhibitors are described in literature, but only a selection of inhibitor classes was investigated in this thesis (Figure 1.6). In the following, the relevant inhibitor classes are listed and briefly described: 1) Boronic acid inhibitors, including the Bortezomib inhibitor with a lead in anti-cancer therapy over the last years, 2) Epoxyketone inhibitors that are a highly potent and customizable class of inhibitors, and 3) $\alpha$-Ketoaldehydes, which were discovered in the 1990s but neglected for several years because their benefit over other inhibitor classes was not understood at that time.<smiles>CC(C)CC(NC(=O)C(Cc1ccccc1)NC(=O)c1cnccn1)B(O)O</smiles>

Bortezomib<smiles>CC(C)CC(NC(=O)CNC(=O)c1cc(Cl)ccc1Cl)B(O)O</smiles>

Ixazomib<smiles>CC(C)CC(NC(=O)C(NC(=O)c1cccc(-c2ccccc2)n1)C(C)O)B(O)O</smiles>

Delanzomib<smiles>COCC(NC(=O)c1cnc(C)s1)C(=O)NC(Cc1ccccc1)C(=O)C1(C)CO1</smiles>

Oprozomib<smiles>CCC(C)C(NC(=O)C(C(C)CC)N(C)C(C)=O)C(=O)NC(C(=O)NC(CC(C)O)C(=O)C1(C)CO1)C(C)O</smiles>

Epoxomicin<smiles>CC(C)CCCCC(=O)NC(CO)C(=O)NC(CC(C)O)C(=O)C1(CO)CP1</smiles>

Dihydroeponemycin<smiles>CC(C)CC(NC(=O)[C@H](CC(C)C)NC(=O)OCc1ccccc1)C(=O)NC(Cc1ccc(O)cc1)C(=O)C=O</smiles>

Z-LLY-Ketoaldehyde

Figure 1.6: Chemical structures of proteasome inhibitors. Chemical structures of the boronic acid inhibitors Bortezomib, Ixazomib and Delanzomib. Oprozomib, Dihydroeponemycin and Epoxomicin belong to the group of $\alpha, \beta$-epoxyketone inhibitors. Z-LLY-Ketoaldehyde represents the $\alpha$-ketoaldehyde inhibitors. 


\subsubsection{Boronic Acid Inhibitors}

Bortezomib, along with other boronic inhibitors is highly potent and inactivates the proteasomal active site by a covalent but slowly reversible binding mode. Boronic acid inhibitors covalently react with the nucleophilic Thr1OY of the $20 \mathrm{~S}$ proteasome proteolytically active subunits. Bortezomib was developed after successful proteasome inhibition was shown for the natural agent lactacystin and synthetic aldehyde inhibitors. Bortezomib is based on the substitution of the aldehyde with boronic acid to gain a compound which forms a covalent, reversible complex and exhibits an improved selectivity for proteasomal active sites compared to corresponding aldehydes ${ }^{64}$.

Bortezomib was the first proteasome inhibitor to enter clinical trials and to be approved for cancer therapy, but despite prolonging the lifetime of multiple myeloma patients, Bortezomib shows severe side effects like neuropathy. In addition, patients often develop resistance and treatment requires an intravenous or subcutaneous injection, which is not ideal. Still, the positive clinical outcome of Bortezomib treatment provided a motivation for the discovery and development of next-generation proteasome inhibitors with higher efficiency and enhanced tolerability.

One of the next-generation boronic acid inhibitors is an orally bioavailable proteasome inhibitor, Ixazomib (MLN2238). Approved by the FDA in 2015, Ixazomib is used for the treatment of multiple myeloma patients with selectivity and potency similar to that of Bortezomib. This reversible proteasome inhibitor preferentially binds to and inhibits the $\beta 5$ active site of the $20 \mathrm{~S}$ proteasome, whereby at higher inhibitor concentrations Ixazomib also inhibits the $\beta 1$ and $\beta 2$ activity ${ }^{65,66}$.

A third boronic proteasome inhibitor, Delanzomib (CEP-18770), is currently in clinical trials. Delanzomib is orally deliverable and has been investigated in Phase I clinical trials for treatment of solid tumors, leukemia and non-Hodgkin's lymphoma ${ }^{67}$. Delanzomib was developed to inhibit the $\beta 5$ chymotryptic-like site of the proteasome ${ }^{68}$. However, it also co-inhibits the caspase-like site $(\beta 1)$ in a concentration-dependent manner ${ }^{69}$.

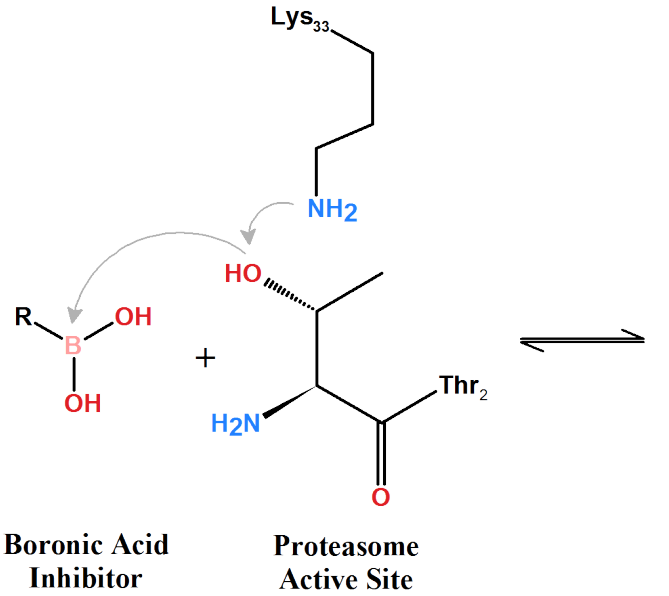

Inhibitor

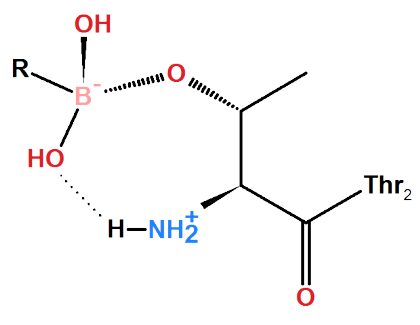

Boronate
Figure 1.7: Mechanism proposed for boronic acid inhibitors. Schematic representation of the inhibition mechanism of boronic acid inhibitors. 


\subsubsection{Epoxyketone Inhibitors}

The epoxyketone class of inhibitors are known for their specificity and potency, with no evidence of off-target effects. The first structural analysis of epoxyketone inhibition was carried out in S. cerevisiae $20 \mathrm{~S}$ proteasomes. These studies proposed a possible mode of proteasome inhibition by the epoxyketone inhibitor Epoxomicin ${ }^{70}$. The inhibitor binds to the Thr1 in the $\beta 5$, chymotryptic-like active site by a formation of a six-membered morpholine ring linkage. The morpholine 6-ring structure formation is described as a 2-step process (Figure 1.8). First, activation of the Threonyl O $\curlyvee$ occures either via the Nterminal group of Thr1 or via a neighboring water molecule found in all active sites of the $20 \mathrm{~S}$ proteasome. A nucleophilic attack of the Thr1O $\gamma$ on the $\alpha, \beta$-epoxyketone's carbonyl carbon forms a hemiacetal. Next, the free $\alpha$-amino group of Thr1 opens up the epoxide ring and intramolecular cyclization takes place. The resulting 1,6-morpholino ring closure is formed, irreversibly inhibiting the active site.

Carfilzomib and its orally bioavailable analog ONX-0912 are two $\alpha^{\prime}, \beta^{\prime}$-epoxyketone inhibitors that are approved or in clinical trials by the FDA, respectively ${ }^{71}$. Carfilzomib is equally potent to the boronic acid inhibitor Bortezomib but more selective for the chymotryptic-like site of the catalytic core. The specificity and relatively simple synthesis made epoxyketone inhibitors a popular choice for the synthesis of many modifications with the result of hundreds of epoxyketones, including many subunit-selective and activity-based inhibitors ${ }^{72}$.
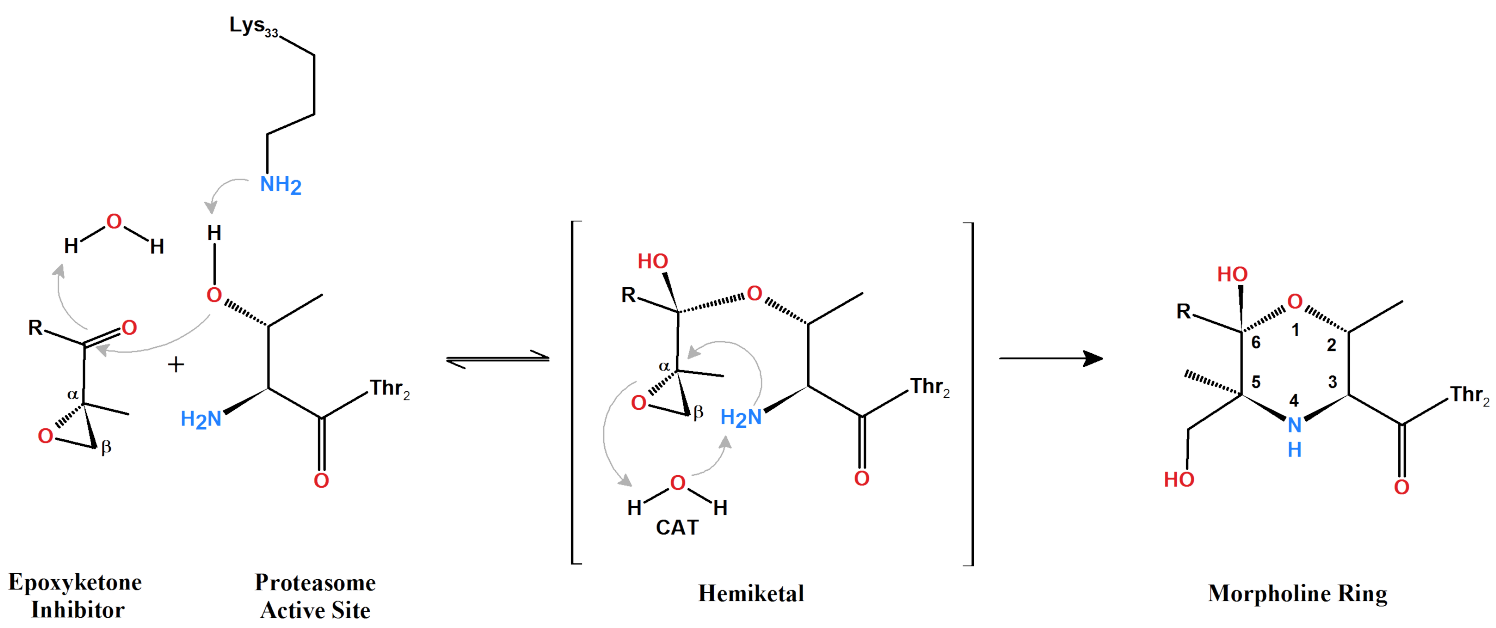

Figure 1.8: Mechanism proposed for $\alpha, \beta$-epoxyketone inhibitors. Schematic representation of the proposed inhibition mechanism of $\alpha, \beta$-epoxyketone inhibitors.

\subsubsection{Ketoaldehyde Inhibitors}

a-Ketoaldehyde inhibitors were first discovered in 1990, but only in 2011 first structural studies of the yeast $20 \mathrm{~S}$ proteasome in complex with a ketoaldehyde revealed details 
about the mode of action ${ }^{73}$. Similar to epoxyketone inhibitors, a ring structure is formed upon inhibition, but unlike epoxyketones, the binding of an $\alpha$-Ketoaldehyde is proposed to result in a 5,6-dihydro- $2 H$-1,4-oxazine ring closure including a hemiketal and an imine bond (Schiff base). Formation of the hemiketal after the nucleophilic attack of Thr1 $\mathrm{O} \gamma$ proceeds as described for epoxyketone inhibition, but the second step encompasses a nucleophilic attack on Thr1N on the aldehyde carbon and a formation of a tetrahedral carbinolamine before releasing a water molecule and forming the rigid 6-membered ring. All steps towards the ring-formation are reversible, giving this class of inhibitors completely different pharmacological properties than those of irreversible epoxyketone morpholine-linkage inhibitors.

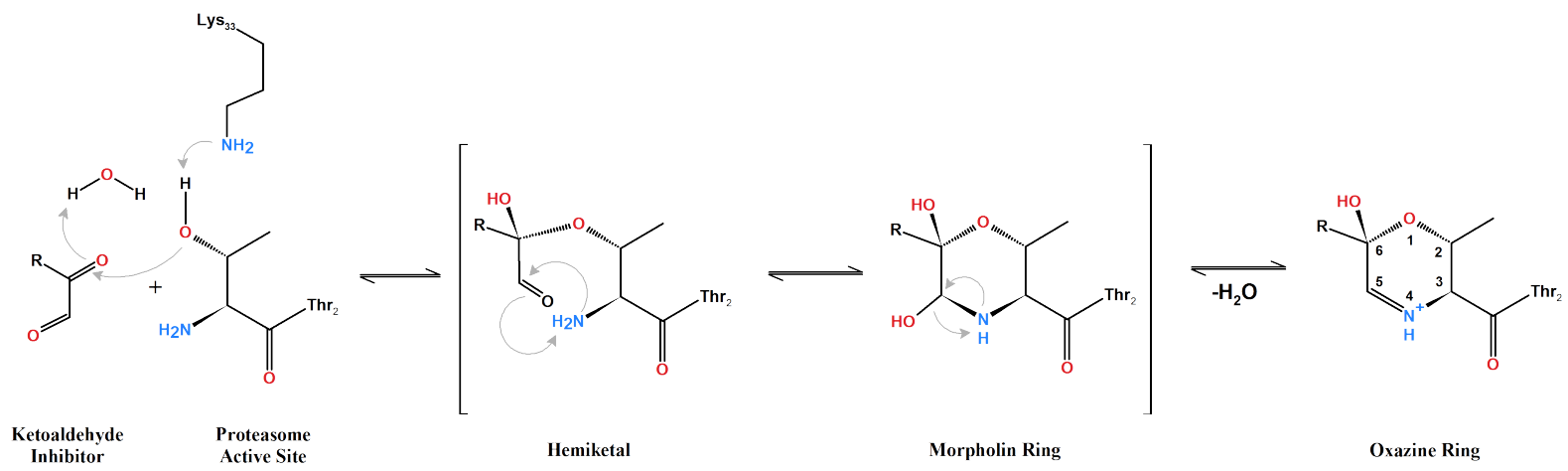

Figure 1.9: Mechanism proposed for $\alpha$-ketoaldehyde inhibitors. Schematic representation of the proposed inhibition mechanism of $\alpha$-ketoaldehyde inhibitors.

\subsubsection{Recent Advances in Proteasome Inhibitor Development}

Most of the proteasome inhibitors which are currently used as chemotherapeutics, or are in development are peptide-based covalent inhibitors. These compounds exert their inhibitory activity by a covalent interaction with the active site Thr1 $\mathrm{O} \gamma$ of the $\beta$-subunit. Covalent inhibitors are highly reactive, which leads to off-target interactions. In addition, the tissue distribution is very low due to slow dissociation kinetics, often inducing apoptosis and cell death in vivo of non-cancerous cells ${ }^{74,75}$.

To overcome the disadvantages related to covalent inhibition, a concerted effort was put into development of noncovalent proteasome inhibitors, which bind the proteasome reversibly and time-limited. A number of classes of substrate-competitive and noncovalent proteasome inhibitors were identified and tested. These classes include Benzylamides, Oxadiazoles, and various natural products ${ }^{76,77,78,79,80,81}$. For the development of new peptide-based noncovalent inhibitors extensive work has been carried out: New inhibitors were synthesized based on high-throughput screening of libraries including several hundred thousand compounds ${ }^{82}$.

In 2000, scientists discovered the natural product TMC-95A and its diastereomeres B, C 
and D from Angiospora montagnei, TMC-95A demonstrated a selective and competitive inhibition of the proteolytic activity of the $20 \mathrm{~S}$ proteasome in a low molecular dose ${ }^{83,80}$. These natural products were characterized as novel cyclic peptides, consisting of a heterocyclic ring system. TMC-95A inhibits all three catalytic sites of the 20S proteasome, shown by crystallographic studies ${ }^{84}$. The mode of inhibition was described as noncovalent without modification of the N-terminal threonine upon inhibition. This noncovalent inhibitor binding is carried out by a specific network of hydrogens bonds. These interactions are between strictly conserved residues of the active sites with main-chain atoms of the inhibitor. TMC-95A adopts an antiparallel $\beta$-sheet structure with the peptide backbone as previously reported for $\alpha^{\prime}, \beta^{\prime}$-epoxyketone and aldehyde inhibitors ${ }^{16,24,80,83}$. TMC-95A is favored over flexible ligands because its rigid ring structure prevents the inhibitor from structural rearrangements upon binding.

Currently many details of proteasome inhibition are known and a number of selective and potent inhibitors have been synthesized. A drug candidate with the optimal characteristics has not yet been found or produced. Results from biological assays as well as structural studies will help to improve existing inhibitors or to design new compounds that may provide better chemotherapeutic treatment solutions in the future.

\subsubsection{Biological Implication of Proteasome Inhibition}

Many cellular pathways are dependent on correct proteasome function. Several proteasomal substrates have essential tasks in cell-cycle regulation. Processing of these substrates by the proteasome are therefore essential. Important complexes involved in cell-cycle progression that depend on proteasome function are the cyclin-dependent kinase (CDK) complexes. CDK activity is regulated by short-living proteins, namely cyclins, which act at various stages of the cell cycle. The rapid turnover of cyclins is carried out by the UPS and only by an ordered and timed degradation of these small regulators can the continued growth of cells be sustained ${ }^{85,86}$. Inhibition of the proteasome-mediated degradation of the cyclin proteins therefore results in reduced cell growth.

Furthermore, the proteasome controls apoptosis by affecting the nuclear factor of $x \mathrm{~B}$ $(\mathrm{Nf}-\varkappa \mathrm{B})$. Nf- $\chi \mathrm{B}$ transcription factors are bound to a specific inhibitor protein, $\mathrm{I} \varkappa \mathrm{B}$, inactivating it and preventing it from activating transcription of its target genes. Upon stress, such as chemotherapy, radiation, viral infection and growth factors, $\mathrm{I} \chi \mathrm{B}$ is phosphorylated and subsequently degraded by the $26 \mathrm{~S}$ proteasome ${ }^{87,88}$, releasing $\mathrm{Nf}-\varkappa \mathrm{B}$. Nf- $\chi \mathrm{B}$ then translocates from the cytoplasm into the nucleus to activate transcription of a series of factors that promote cell growth and differentiation, and prevent apoptosis. Importantly, $\mathrm{Nf}-\chi \mathrm{B}$ initiates its own transcription and the transcription of its inhibitor $\mathrm{I} \chi \mathrm{B}$, maintaining its own activity by positive feedback ${ }^{57}$. By an inhibition of the proteolytic activity 
of the proteasome, $\mathrm{I} \chi \mathrm{B}$ is stabilized in the cell and cancer cells are more vulnerable to chemotherapeutic or radiation treatments.

Proteasome function also influences the activation of the tumor suppressor p $53^{89}$. p53 is a short-lived protein with low quantities in the healthy eukaryotic cell. Cellular stresses such as chemical- or radiation-induced DNA damage, oxidative stress or oncogene activation cause p53 to accumulate in the cell, which triggers diverse cellular responses such as cell-cycle arrest. The cell efficiently regulates the cellular stress response by a feedback regulation via the p53-induced expression of MDM2 which then transports p53 from the nucleus to the cytoplasm and ubiquitinates p53 for rapid proteasomal degradation $^{90}$. Upon proteasome inhibition, p53 becomes activated and stimulates p53-mediated tumor-suppressor activity that result in apoptosis and senescence. 


\subsection{An Introduction to X-ray Crystallography and Single Particle Electron Cryomicroscopy}

Understanding the chemical and biological functions, and mechanisms of macromolecular complexes often requires structural data with details at atomic resolution. Developments in technology and methodology over the last 20 years have paved the road to narrow the gap between two effective methods to study the structure of macromolecular machines: X-ray crystallography and single particle electron cryomicroscopy. In the present thesis both methods were utilized to analyze proteasome inhibition of either the 20S proteasome or the $26 \mathrm{~S}$ holoenzyme. In the following section, a short introduction to both techniques is provided and the advantages and disadvantages of the techniques is presented.

\subsubsection{X-ray Crystallography}

X-ray crystallography is by far the oldest technique to investigate the structures of single molecules as well as macromolecular complexes. The discovery of X-rays by Wilhelm Conrad Roentgen in 1895 lead the way to its application in crystallography in the beginning of the 20th century and its first application in synchrotron radiation in the mid-1970s ${ }^{91}$. In 1958, John Kendrew unveiled the first protein structure, the structure of Myoglobin, a milestone in the history of structural biology ${ }^{92}$. Only two years later, Perutz published the structure of Hemoglobin ${ }^{93}$. These milestones were achieved because a group of crystallographers around Perutz discovered the "method of isomorphus replacement" by which for the first time the crystallographic phase problem was overcome. Perutz discovered that heavy atoms could be attached to protein molecules and by this, the diffraction pattern would differ from crystals without heavy atoms. With this, phases of the reflections could be inferred for the first time ${ }^{94,95}$.

In diffraction experiments, intensities of waves scattered from planes in the crystal are measured. The amplitude of the wave is proportional to the square root of the intensity measured on the detector. To calculate the electron density at a position in the unit cell, a summation of all planes is performed. Here, a certain electron density is the sum of contributions to the point of waves scattered from a plane, whose amplitude depends on the number of electrons in the plane, added with the correct relative phase relationship. This can be written mathematically as:

$$
p(x y z)=1 / V \sum\left|F_{h k l}\right| \exp \left(i \alpha_{h k l}\right) \exp (-2 \pi i h x+k y+l z)
$$


where $V$ is the volume of the unit cell, $h k l$ is the crystal plane, the position in the unit cell is described as $x y z$ and $a_{h k l}$ is the phase associated with the amplitude $\left|F_{h k l}\right|^{96}$. The amplitudes can be measured but the phases are lost, which is known as the "phase problem".

For successful data collection a combination of the best possible instrumentation and a data collection strategy is needed. Today, diffraction patterns are measured at modern beamlines in synchrotron facilities with a very strong beam intensity, resulting in short exposure times. Data sets are collected with the oscillation photography method. The axes of the crystal are carefully aligned to the beam based on pre-screening of the crystal and the crystal is rotated around the set oscillation axis with diffraction patterns collected at defined angular distances.

Diffraction of protein crystals relies almost exclusively on scattering of X-rays by the electrons in the molecule resulting in spots measured on the detector during data collection. These spots have to be interpreted and incorporated into an overall dataset by scaling and merging. The amplitudes of interfering X-ray waves can be identified and the unit cell dimensions, crystal symmetry, and information of the molecular packing can be determined from the diffraction pattern intensities.

For calculating an electron density map, phase information has to be obtained. In addition to the method of isomorphus replacement introduced by Perutz, several experimental procedures to phase observed structure factor amplitudes were developed. One common way to determine phases is molecular replacement ${ }^{97}$. Here, for example a reasonable homology model, can be used to provide an initial estimate for the phases. Besides this, a number of "direct methods" exist. Ab initio phasing is used when no phases from a structurally similar model are available, or if one wants to circumvent any kind of model bias by molecular replacement. To date, a standard $a b$ initio method is still the Single or Multiple Isomorphous Replacement (SIR, MIR) method. Here, diffraction data from the native crystal and one or more crystals with derivatives, which produces a different diffraction pattern, is collected ${ }^{98}$. Another approach is Single and Multiple Anomalous Dispersion (SAD, MAD) ${ }^{99,100}$. Data is collected from a single crystal at several wavelengths, one wavelength at the absorption peak, one at the point of inflection and one at a remote wavelength. Whereas with SIR and MIR the protein phases are estimated from the additional scattering of the heavy metal atom crystals, in SAD and MAD the phases are calculated from wavelength-dependent quantitative differences in the anomalous scattering contribution of certain atoms contained within crystals.

Previously it was shown that phases for macromolecular complexes could be solved with anomalous scattering. The 30S ribosomal subunit from B. Stearothermophilus was solved by using the heavy atoms, osmium and lutetium at $5.5 \AA$ resolution ${ }^{101}$. Similarly the fully assembled $T$. thermophilus $70 \mathrm{~S}$ ribosome was phased with the anomalous scatterer iridium and solved to $7.8 \AA$ resolution ${ }^{102}$. 
Concluding the general X-ray crystallographic workflow, the determination of the initial phases is followed by the refinement of the phases, ending with a correct structural model. A model of the molecule is then built that agrees with both the information from diffraction in addition to biochemical knowledge.

Until today, X-ray crystallography has become the dominating method to determine three-dimensional structures of biological macromolecules. Synchrotron facilities that provide brilliant X-ray sources have extremely fast data-collection times and highly developed crystallographic methods form the basis to study macromolecules at very high resolution. Besides data collection and interpretation, one step for determining a three dimensional structure is fundamental: the crystallization of protein. The outcome of the crystallization procedure is determined by the choice of sample, optimization of the crystallization condition and post-crystallization treatments.

For the crystallization of macromolecular samples, the choice of the sample is often determined before starting with sample purification, or while searching for a suitable purification protocol. The initial step for crystallization is done when a reproducible purification strategy for the sample of choice is found and the soluble macromolecule can be prepared in viable quantities. For crystallization, the sample is then transferred into a solution, called mother liquor, in which it forms crystals. Finding a crystallization condition in which the flexible macromolecules arrange themselves in an ordered fashion can take time and often results in protein crystals which are fragile and need a complex postcrystallization treatment. Crystallization trials are performed in conditions that vary in buffer substance, $\mathrm{pH}$, ions and precipitants. Besides the crystallization solution a number of factors are decisive for the optimal crystallization condition, including sample concentration, temperature and crystallization method. Most protein crystals are produced by the vapour diffusion method, either in hanging or sitting drop crystallization plates at temperatures ranging from room temperature $\left(20-25^{\circ} \mathrm{C}\right)$ to temperatures close to the freezing point of water $\left(0^{\circ} \mathrm{C}\right)$. The setup for the vapour diffusion methods is relatively simple: A drop containing the protein sample is equilibrated against a large reservoir of mother liquor. Volatile substances can then diffuse between drop and reservoir, reaching an equilibrium in which the protein will crystallize. The absence of crystals and a clear drop indicates that the equilibration is still ongoing or the sample concentration is too low. When the drop shows precipitate but no crystals, the sample concentration might be too high. The protein crystals obtained are mostly fragile and/or they contain ordered molecules but also disordered solvent in between. The crystal volume occupied by solvent molecules is at about 40-60\% depending on the crystal ${ }^{103}$. With post-crystallization treatments the volume of solvents can be reduced, and the crystal lattice becomes more ordered or the packing is more dense. This can even change the crystallographic space group. These rearrangements reinforce and form new crystal contacts, resulting in better diffracting crystals. The most effective post-crystallization protocol must be evaluated for 
every crystal condition. Mostly it encompasses an increase or introduction of precipitant and/or the addition of small molecules which stabilize the crystal internally. Crystal dehydration can lead to remarkable improvements in crystal diffraction ${ }^{104}$.

Since collection of X-ray data is carried out using a high intensity beam, the energy can cause severe heating and radiation damage on the crystal. To increase the lifetime of crystals in the X-ray beam it is an advantage to cool the crystals to very low temperatures $\left(100 \mathrm{~K} /-173{ }^{\circ} \mathrm{C}\right)$, the crystals must be protected from ice formation by using cryo-protectants. These cryo-protectants include Glycerol, MPD, low molecular weight PEGs and oils. The crystals are transfered to the cryo-protectant for varying time spans between a few seconds and hours. The crystals are then frozen in liquid nitrogen $\left(\mathrm{lN}_{2}\right)$ prior to X-ray measurements. An overall workflow of X-ray crystallography is presented in Figure 1.10.

\subsubsection{Single Particle Electron Cryomicroscopy}

Electron microscopy is the youngest among all other methods to determine three dimensional structures of protein complexes. In 1872, Ernst Abbe formulated his wave theory of microscopic imaging and about 60 years later the first electron microscope was built by Ernst Ruska and Max Knoll ${ }^{105}$. Historically, electron microscopes were developed to overcome the limited resolution in light microscopes, imposed by the wavelength of visible light. Due to the small wavelength of electrons electron microscopes are capable of imaging at a higher resolution.

Using electron microscopy, a broad specimen range from the atomic to tissue scale objects can be imaged, providing snapshots of biological samples and cells close to their native states. For isolated macromolecular complexes the method of choice is the single particle electron cryomicroscopy approach using a transmission electron microscope (TEM). The TEM has a very similar setup to a light microscope, whereby the image is formed by transmitting electrons and not light. Those electrons interacting with the sample contribute to a phase contrast image, the electron micrograph. Phase contrast imaging is the highest resolution imaging technique ever developed, and can allow for resolutions of less than $1 \AA$.

Macromolecular machines are important in all living cells and with improving imaging and image processing methods, electron microscopy is now a standard technique to study macromolecular assemblies. The method became popular when it was possible to structurally study macromolecular complexes which are resistant to crystallization, albeit at lower resolution. Over the years, technological advances in sample preparation, instrumentation and computational analysis made electron microscopy a benchmark for structural studies. 
Structural investigation of macromolecular complexes starts with a specialized sample preparation method. The purified protein sample is usually applied to an EM grid covered with a thin holey carbon film and blotted by a filter paper to remove excess solution to form a thin liquid layer. The sample is then rapidly vitrified in liquid ethane ${ }^{106}$ to bring all particles to a solid state and to prevent dehydration within the vacuum of an electron microscope. Freezing also reduces the effects of electron beam damage ${ }^{107}$. Next, the images, called electron micrographs, are collected to capture 2-dimensional projections of single particles in spacial orientations. Due to low dose exposure to reduce beam damage, the 2D micrographs have a poor image contrast. To reduce the noise, a high number of individual particles in the same orientation are averaged using software that aligns and merges the data. An initial 3D volume is constructed from many averaged images and the map is then iteratively refined and validated to become the final 3D map. Subsequently, the model is built and the quality of the map is then evaluated.

Recent technical advances in single particle cryo-EM allow structures of macromolecular machines to be determined in the resolution range between 2.5 to $5 \AA$. For example, ribosomes from human pathogens ${ }^{108}$ or ribosome-protein complexes provide insight to their functions. Simultaneously, technical improvements in cryo-EM led to structures of smaller complexes with molecular masses smaller than 200 kilodaltons $(\mathrm{kDa})$ to be determined ${ }^{109}$. An idealized single particle electron cryomicroscopy workflow is presented in Figure 1.10.

\subsubsection{Advantages and Disadvantages}

In the last years, the major advances achieved in all aspects of structural biology are numerous. Sample preparation, crystallization, access to synchrotron facilities, high-end microscopes and high-speed computing enable multiple solutions to gain structural information of many microscopic objects. Working with X-ray crystallography and single particle electron cryomicroscopy means working with two diffraction-based techniques that bring along their own benefits and limitations.

A decisive factor when comparing these two techniques is the sample amount. For many protein complexes scientists struggle to purify large quantities, because the sample is labile or the purification protocol is not mature. For EM, only relatively low amounts of sample are needed, whereas crystallization often requires amounts in the milligram quantity range.

Both X-ray and electron beams cause radiation damage to biological samples. In X-ray crystallography some crystals tolerate high total doses because of their size and/or packing within the crystal. In EM, the total electron dosage to image the single particles has to be set to a minimum to preserve the structural information. As a consequence, low 
dose imaging results in a poor signal-to-noise ratio making it is difficult to obtain good quality data.

X-ray diffraction reveals its major limitation when studying macromolecular complexes: crystallization of dynamic complexes is challenging and phasing represents the common bottleneck in crystallography. Even with outstanding samples, a successful analysis by X-ray crystallography is limited by the production of crystals and finally by the crystal quality which is still a trial and error approach. Nevertheless, in the last ten years an unprecedented increase in the number of new crystal structures in the Protein Database (PDB) is apparent.

Electron cryomicroscopy is suitable for large (>100 kDa) macromolecular complexes. Cryo-EM often struggles with problems in image analysis, which is mostly dependent on the sample quality. However, electron cryomicroscopy represents a powerful tool to study the structure and dynamics of macromolecular complexes at intermediate resolutions between 10 and $30 \AA$, while resolutions around $3 \AA$ can also be achieved. The resolution limit around $3 \AA$ was reached with macromolecular machines like polymerases, ribosomes and viruses from various organisms.

Moreover, hybrid structural approaches are also common. Cryo-EM and X-ray crystallography are complementary techniques; often atomic models from crystallographic studies are combined with electron microscopic data at medium resolution. 


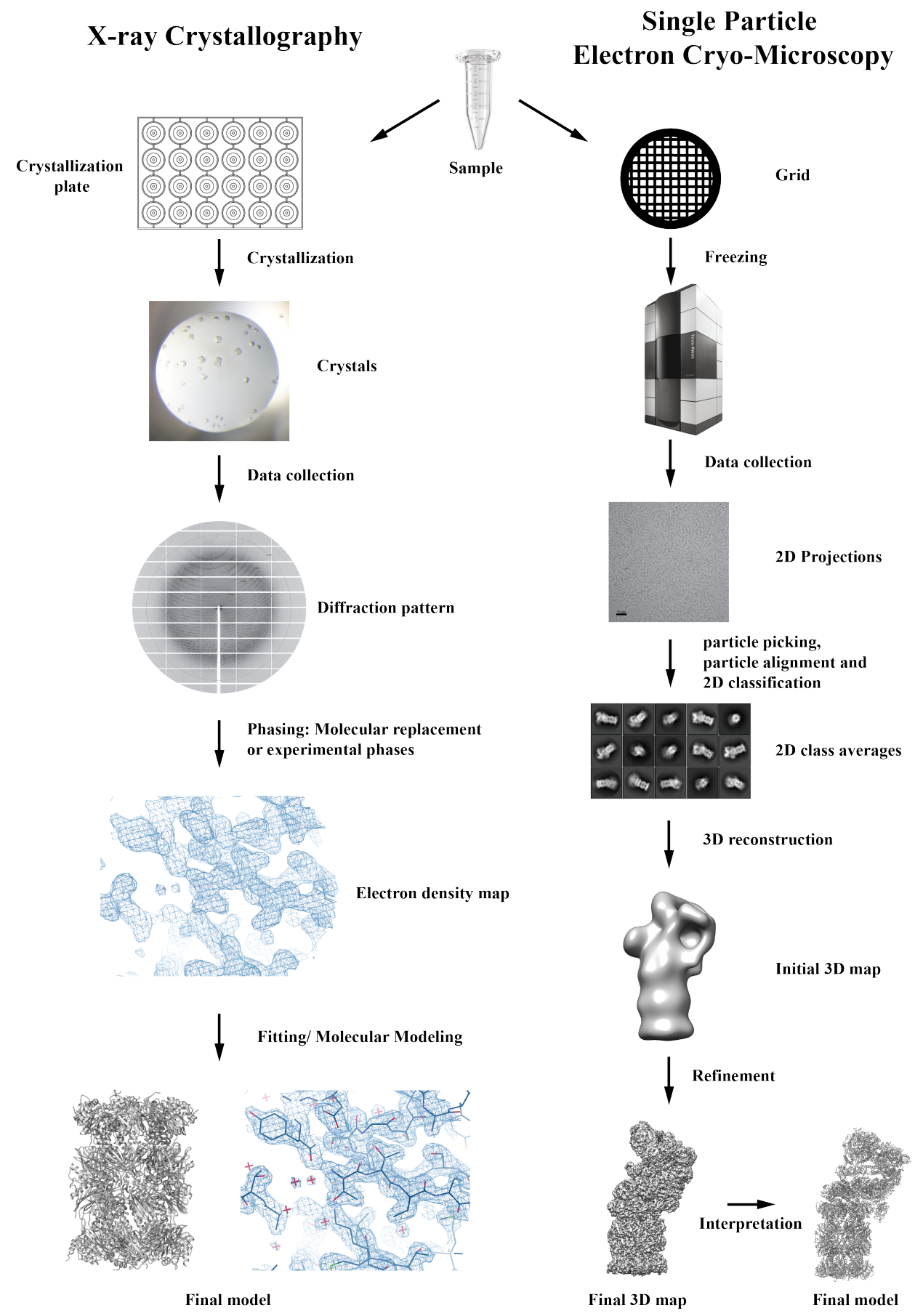

Figure 1.10: The overall X-ray crystallography and single particle cryo-EM workflow, from protein sample to 3D model. Schematic figure showing the workflow for macromolecular structure determination by X-ray crystallography and Cryo-EM. (1) X-ray crystallography: Diffraction images are obtained from protein crystals. These images encode the structural information. With the help of Fourier transformations, the information is translated into the molecular real space. Since the phases are not directly accessible so-called phasing approaches have to be performed. With further experimental data or by molecular replacement calculations these phases are obtained. Once initial phases are obtained, electron density maps are calculated. Molecular model building and structural refinement form the final steps of x-ray crystallographic model building. (2) Cryo-EM: A single-particle project starts with specimen preparation and the collection of EM images. The single particles are isolated and $2 \mathrm{D}$ analysis is performed. Next, an initial 3D map is calculated and iteratively refined. The final map is used to build a $3 \mathrm{D}$ model of the macromolecule. 


\subsection{Objectives}

The aim of this thesis is to structurally characterize the inhibition of the human proteasome. During the course of this thesis, special attention was paid to the development of new methods and improvement of known methods to find ways for screening and characterizing the inhibition of the human $20 \mathrm{~S}$ and $26 \mathrm{~S}$ proteasome.

To elucidate structures of the human 20S proteasome, a reproducible, large-scale method to purify proteasomes from HeLa cytoplasmic extract was needed. Hence, a great effort was spent on the development of a gentle, chromatography-free purification strategy for human proteasomes. A robust pipeline for the production and crystallization of human $20 \mathrm{~S}$ proteasomes could be established. The crystallization procedure reproducibly yielded $20 \mathrm{~S}$ proteasome crystals in high abundance and allowed the analysis of native human $20 \mathrm{~S}$ proteasome and in complex with cancer therapeutics in greater detail.

In the second part of this thesis, the structural implications of $20 \mathrm{~S}$ core particle inhibition on the human $26 \mathrm{~S}$ holoenzyme were studied. The novel purification strategy was transferred to human $26 \mathrm{~S}$ proteasomes, yielding high amounts of $26 \mathrm{~S}$ proteasome complexes. Structures of human $26 \mathrm{~S}$ proteasomes with and without inhibitors were analyzed by single particle electron cryomicroscopy and the structural differences were investigated. The visualization of changes in the structure of the human 26S proteasome upon drug binding then allowed to formulate a hypothesis for future proteasome inhibitor design. 


\section{Chapter 2}

\section{Materials and Methods}

\subsection{Materials}

\subsubsection{Software}

Table 2.1: Software used in this thesis.

\begin{tabular}{l|l}
\hline Software & Source \\
\hline Amira 4.1 & https://www.fei.com/software \\
Adobe Creative Suite 5 & http://www.adobe.com/de/creativecloud.html \\
CCP4 & http://www.ccp4.ac.uk \\
Cow software suite & Group of Prof. Dr. Stark, Göttingen \\
COOT & https://www2.mrc-lmb.cam.ac.uk/personal/pemsley/coot ${ }^{111}$ \\
Gautomatch & http://www.mrc-lmb.cam.ac.uk/kzhang \\
Gctf & http://www.mrc-lmb.cam.ac.uk/kzhang ${ }^{112}$ \\
Imagic & https://www.imagescience.de/imagic_em.html ${ }^{113}$ \\
JLigand & http://www.ysbl.york.ac.uk/mxstat/JLigand ${ }^{114}$ \\
KaleidaGraph 4.03 & http://www.synergy.com/wordpress_650164087 \\
Orca 3.0.3 & https://orcaforum.cec.mpg.de/ \\
OriginPro 9.1 & http://www.originlab.de/Origin \\
Phenix & https://www.phenix-online.org \\
Python 2.7 & https://www.python.org \\
PyMOL & https://www.pymol.org \\
RELION 1.2 & http://www2.mrc-lmb.cam.ac.uk/relion ${ }^{115}$ \\
ResMap & http://resmap.sourceforge.net \\
Simple PRIME & http://simplecryoem.com \\
UCSF Chimera & http://www.cgl.ucsf.edu/chimera ${ }^{117}$ \\
\hline
\end{tabular}




\subsubsection{Special Equipment}

Table 2.2: Machines and special equipment used in this thesis.

\begin{tabular}{l|l}
\hline Machine/Equipment & Manufacturer \\
\hline ActiLoops & Molecular dimensions \\
Äkta Prime & GE Healthcare \\
Balances & Sartorius \\
Centrifuge LYNX 6000 & Thermo Scientific \\
Copper EM grids & Plano \\
Cryschem M Plate, 24-well & Hampton Research \\
Crystal Clear Sealing Tape & Jena Bioscience \\
Crystalgen Plate 24-well, pregreased & Jena Bioscience \\
Fiberlite rotor F14, F21, F35L & Thermo Scientific \\
FluoroMax-4 fluorescence spectrophotometer & Horiba Scientific \\
Gradient Master ip & Biocomp \\
Incubator Eco-Line E100 & RUMED \\
Incubator Multitron Pro Shaker & Infors HT \\
Leica EM GP & Leica \\
LithoLoops & Molecular dimensions \\
Micro-Ultracentrifuge MX 150+ & Sorvall \\
Orbital Shaker RS0S20 & Phoenix Instrument \\
Photometer Lambda Bio X & Perkin Elmer \\
Plate Sealer ALPS 3000 & Thermo Scientific \\
Pipetting Robot Microlab Star LET & Hamilton \\
Quantifoil grid R3.5/1 Cu 200 mesh & Quantifoil Micro Tools GmBH \\
Rotor TH660 & Sorvall \\
Rotor S140AT & Thermo Scientific \\
Rotor SW40 & Beckmann Coulter \\
Siliconized Glass Cover Slides & Hampton Research \\
Stereo microscopes M125 & Leica \\
TEM CM200 FEG & Philips \\
TEM Titan Krios & Ultracentrifuge WX Ultra 80, 90 \\
Vitrobot & Tebra Micro Desalt Spin Columns \\
\hline
\end{tabular}




\subsubsection{Chemicals}

Table 2.3: Chemicals, Substrates, Enzymes and Antibodies used in this thesis.

\begin{tabular}{|c|c|}
\hline Chemicals & Supplier \\
\hline 2-methyl-2,4-pentanediol (MPD) & Sigma-Aldrich \\
\hline 1,4-Dithiothreitol (DTT) & Roth \\
\hline Aspartate & Sigma-Aldrich \\
\hline$\beta$-Gycerophosphate disodium salt hydrate & Sigma-Aldrich \\
\hline Benzamidine Hydrochloride hydrate & Sigma-Aldrich \\
\hline Bis[sulfosuccinimidyl] suberate (BS3) & Thermo Scientific \\
\hline Bis(2-hydroxyethyl)amino-tris(hydroxymethyl)methan & Sigma-Aldrich \\
\hline Bovine serum albumin (BSA) & Sigma-Aldrich \\
\hline Bradford Assay reagent & BioRad \\
\hline Coomassie Brilliant Blue R-250 & BioRad \\
\hline Dimethylsulphoxide (DMSO) & AppliChem \\
\hline Dinatriumhydrogen phosphate $\left(\mathrm{Na}_{2} \mathrm{HPO}_{4}\right)$ & Merck \\
\hline Dipotassium phosphate $\left(\mathrm{K}_{2} \mathrm{HPO}_{4}\right)$ & Merck \\
\hline Ethanol & Merck \\
\hline Ethylenediaminetetraacetic acid (EDTA) & Merck \\
\hline Glucose & Merck \\
\hline Glutaraldehyde & Science Services \\
\hline Glycerol & Merck \\
\hline Iodoacetamide & Sigma-Aldrich \\
\hline Isopropanol & Merck \\
\hline Lauryl Maltose Neopentyl Glycol & Anatrace \\
\hline Magnesium chloride & Merck \\
\hline Magnesium acetate & Merck \\
\hline N-ethylmaleimide & Sigma-Aldrich \\
\hline Octyl Glucose Neopentyl Glycol & Anatrace \\
\hline Phosphoenolpyruvic Acid Tris(cyclohexylammonium) & \\
\hline Salt Hydrate & TCI \\
\hline Polyethylene glycol (PEG) 400 & Sigma-Aldrich \\
\hline Polyethylene glycol (PEG) 3350 & Sigma-Aldrich \\
\hline Potassium chloride & Merck \\
\hline Potassiumdihydrogen phosphate $\left(\mathrm{KH}_{2} \mathrm{PO}_{4}\right)$ & Merck \\
\hline Protamin sulphate & Sigma-Aldrich \\
\hline Protease inhibitor tablet, EDTA free & Roche \\
\hline Protein Marker & BioRad \\
\hline
\end{tabular}




\begin{tabular}{l|l} 
Pyruvate kinase & Roche \\
Sucrose & Merck \\
Sodium chloride & Merck \\
SDS-PAGE running buffer & Roth \\
Sulfo-MBS & Thermo Scientific \\
& \\
\hline Substrates, enzymes, antibodies & Supplier \\
\hline Bortezomib & Selleckchem \\
Delanzomib & Selleckchem \\
Dihydroeponemycin & APExBIO \\
Epoxomicin & APExBIO \\
Ixazomib & Selleckchem \\
Oprozomib & Selleckchem \\
Suc-LLVY-AMC & Bachem \\
Ubiquitin Antibody & Santa Cruz \\
Z-LLY-Ketoaldehyde & Biotechnology \\
\hline
\end{tabular}




\subsubsection{Buffers}

Table 2.4: Buffers for protein purification, crystallization, crystal stabilization/dehydration and enzymatic assay. Composition of all buffers used for the experiments listed in this thesis.

\begin{tabular}{|c|c|}
\hline Purpose & Composition \\
\hline Purification & $\begin{array}{l}0.05 \mathrm{M} \text { BisTris } \mathrm{pH} 6.5,0.05 \mathrm{M} \mathrm{KCl}, 0.01 \mathrm{M} \mathrm{MgCl}_{2} \text {, } \\
0.01 \mathrm{M} \text {-Glycerophosphate }\end{array}$ \\
\hline Crystallization & $0.1 \mathrm{M}$ BisTris $\mathrm{pH}$ 6.5, $0.2 \mathrm{M} \mathrm{MgCl}_{2}, 10 \%$ (w/v) PEG3350 \\
\hline Crystal stabilization & $0.1 \mathrm{M}$ BisTris pH 6.5, 0.2 $\mathrm{M} \mathrm{MgCl}_{2}, 20 \%$ (w/v) PEG3350 \\
\hline Crystal dehydration & $\begin{array}{l}0.1 \mathrm{M} \text { BisTris } \mathrm{pH} \text { 6.5, } 0.2 \mathrm{M} \mathrm{MgCl}_{2}, 25 \% \text { (w/v) PEG3350, } \\
20 \%(\mathrm{v} / \mathrm{v}) \text { MPD }\end{array}$ \\
\hline Enzyme activity & 0.05 M BisTris pH 6.75, $1 \mathrm{mM}$ EDTA, $0.01 \%$ (w/v) SDS \\
\hline
\end{tabular}




\subsection{Methods}

\subsubsection{Protein Purification}

\subsubsection{Purification of 20S Proteasomes from HeLa cells}

S30 HeLa cytoplasmic extract was prepared by hypotonic lysis according to Dignam et al. ${ }^{118}$, with some minor modifications: after hypotonic lysis and centrifugation to collect nuclei, the supernatant (the crude cytoplasmic extract) was centrifuged at $30.000 \mathrm{x} g$ for 30 minutes at $4{ }^{\circ} \mathrm{C}$ and flash frozen in $40 \mathrm{ml}$ aliquots in liquid nitrogen and stored at $-80{ }^{\circ} \mathrm{C}$ until further use.

The S30 HeLa cytoplasmic extract was thawed in a water bath at $37^{\circ} \mathrm{C}$, supplemented with purification buffer to $1 \mathrm{x}$ concentration from a $10 \mathrm{x}$ stock, followed by the addition of sucrose powder to $20 \%$ (w/v), Octyl Glucose Neopentyl Glycol (OGNG; from a $10 \%(\mathrm{w} / \mathrm{v})$ stock solution in water) to $0.1 \%(\mathrm{w} / \mathrm{v})$, Iodoacetamide to $10 \mathrm{mM}, \mathrm{N}-$ Ethylmaleimide to $10 \mathrm{mM}$ and Benzamidine Chloride to $10 \mathrm{mM}$. The extract was incubated at room temperature on a magnetic stirrer for 30 minutes, followed by incubation at $30{ }^{\circ} \mathrm{C}$ with shaking at $140 \mathrm{rpm}$ for 1 hour. The treated extract was centrifuged at $100.000 \mathrm{x}$ g for 2 hours at $4{ }^{\circ} \mathrm{C}$. After centrifugation, the supernatant was filtered through 3 layers each of cheese cloth and miracloth to obtain a S100 HeLa cytoplasmic extract. The clarified extract is subjected to differential precipitation with Poly Ethylene Glycol400 (PEG; number signifies the mean molecular weight of the PEG polymer). PEG400 is added at a concentration of $20 \%(\mathrm{v} / \mathrm{v})$ to the S100 HeLa cytoplasmic extract under stirring at $4{ }^{\circ} \mathrm{C}$ and incubated for 20 minutes. Precipitated proteins are removed by centrifugation at $30.000 \mathrm{x}$ g for 30 minutes at $4{ }^{\circ} \mathrm{C}$. The supernatant is then precipitated by raising the concentration of PEG400 to $30 \%(\mathrm{v} / \mathrm{v})$ as described above. The precipitate of this step, which contains human $20 \mathrm{~S}$ proteasomes, is recovered by centrifugation at $30.000 \mathrm{x}$ g for 30 minutes at $4{ }^{\circ} \mathrm{C}$ and resuspended in purification buffer containing $2 \%$ $(\mathrm{w} / \mathrm{v})$ sucrose, $10 \mathrm{mM}$ DTT and $0.01 \%$ (w/v) Lauryl Maltose Neopentyl Glycol (LMNG) in an orbital shaker at $18{ }^{\circ} \mathrm{C}$.

The resuspended material is loaded on 10-30 \% (w/v) sucrose gradients in purification buffer containing $5 \mathrm{mM}$ DTT, which are centrifuged at $270.000 \mathrm{x}$ g for 16 hours at $4{ }^{\circ} \mathrm{C}$. Gradients were harvested in $400 \mu \mathrm{l}$ fractions using Äkta Prime. SDS-PAGE ${ }^{119}$ was utilized to identify fractions containing $20 \mathrm{~S}$ proteasomes. Selected fractions were pooled and precipitated by the addition of $40 \%$ (v/v) PEG400. After centrifugation (30.000 x g, 20 minutes), the supernatant was removed and the precipitate was resuspended in Purification buffer containing $5 \%(\mathrm{w} / \mathrm{v})$ sucrose, $10 \mathrm{mM}$ DTT and $0.01 \%(\mathrm{w} / \mathrm{v})$ LMNG. The resuspended material is loaded on linear 10-40\% (w/v) sucrose gradients in Purification 
buffer containing $5 \mathrm{mM}$ DTT, which are centrifuged at $284.000 \mathrm{x} \mathrm{g}$ for 20 hours at $4{ }^{\circ} \mathrm{C}$. Fractions containing 20S proteasomes are yet again identified by SDS-PAGE, precipitated and concentrated by the addition of $40 \%$ PEG400 and resuspended in Purification buffer containing $5 \%(\mathrm{w} / \mathrm{v})$ sucrose, $5 \mathrm{mM}$ DTT and $0.01 \%$ LMNG, yielding the finally purified protein preparation at $13 \mathrm{mg} / \mathrm{ml}$.

Protein concentrations were determined by the Bradford assay (BioRad, Munich, Germany) using BSA as a standard. This procedure reproducibly yields $20 \mathrm{mg}$ purified human 20S proteasomes, starting from $300 \mathrm{ml}$ S100 HeLa cytoplasmic extract at a concentration of $10 \mathrm{mg} / \mathrm{ml}$. The entire purification procedure is concluded within 48 hours yielding crystallization grade protein.

The purification description is according to Schrader at al., $2016^{120}$.

\subsubsection{Purification of 26/30S Proteasomes from HeLa Cells}

Purification of human 26/30S proteasomes was performed according to the recently developed human 20S proteasome protocol, as described above. S30 HeLa cytoplasmic extract is prepared by hypotonic lysis ${ }^{118}$, centrifuged at $30.000 \mathrm{x}$ g for 30 minutes at $4{ }^{\circ} \mathrm{C}$, flash frozen in liquid nitrogen and stored at $-80^{\circ} \mathrm{C}$.

The S30 extract was thawn in a water bath at $37{ }^{\circ} \mathrm{C}$, supplemented with purification buffer to $1 \mathrm{x}$ from a 10x stock, sucrose powder to $20 \%$ (w/v), Octyl Glucose Neopentyl Glycol (OGNG; from a $10 \%$ (w/v) stock solution in water) to $0.1 \%$ (w/v), Iodoacetamide to $10 \mathrm{mM}$, N-Ethylmaleimide to $10 \mathrm{mM}$, Benzamidine Chloride to $10 \mathrm{mM}$ and ATP to $7.5 \mathrm{mM}$. The extract was incubated at room temperature on a magnetic stirrer for 30 minutes, followed by an addition of Dithiothreitol (DTT) powder to $50 \mathrm{mM}$ and a second incubation at room temperature for 30 minutes. The S100 extract was prepared by centrifugation at $100.000 \mathrm{x}$ g for 2 hours at $4{ }^{\circ} \mathrm{C}$ and the supernatant was filtered through 3 layers each of cheese cloth and miracloth.

The S100 extract was processed by two subsequent rounds of precipitation with PEG400. First, PEG400 was added to a concentration of $23 \%(\mathrm{v} / \mathrm{v})$ to the S100 extract at $18{ }^{\circ} \mathrm{C}$ on a magnetic stirrer and incubated for 30 minutes. Second, the supernatant was precipitated by raising the concentration of PEG400 to $30 \%(\mathrm{v} / \mathrm{v})$ as described before. The precipitate contains the human $26 \mathrm{~S} / 30 \mathrm{~S}$ proteasomes and was resuspended with purification buffer supplemented with $7.5 \mathrm{mM}$ ATP, $5 \mathrm{mM}$ DTT and $0.01 \%$ (w/v) Lauryl Maltose Neopentyl Glycol (LMNG) in an orbital shaker at $18^{\circ} \mathrm{C}$. The resuspended material was incubated with an ATP regeneration system (10 mM Sodium Creatine phosphate, $5 \mathrm{\mu g} / \mathrm{mL}$ Creatine kinase) at $30{ }^{\circ} \mathrm{C}$ for 30 minutes.

The sample was loaded on $20 \% / 50 \%$ 2-step sucrose cushions in purification buffer containing $7.5 \mathrm{mM} \mathrm{ATP}$ and $5 \mathrm{mM}$ DTT. The cushions were centrifuged at $260.000 \mathrm{x} \mathrm{g}$ for 14 hours at $4{ }^{\circ} \mathrm{C}$, harvested in $500 \mu \mathrm{l}$ fractions with Äkta PrimeTM (GE Healthcare, 
Munich, Germany) and analyzed by SDS-PAGE to identify fractions containing 26S and $30 \mathrm{~S}$ proteasomes. Fractions were pooled and precipitated by the addition of $40 \%$ (v/v) PEG400 for 30 minutes and after centrifugation (30.000 x g, 30 minutes) the precipitate was resuspended in purification buffer containing $5 \%$ sucrose, $7.5 \mathrm{mM}$ ATP, $5 \mathrm{mM}$ DTT and $0.01 \%(\mathrm{w} / \mathrm{v})$ LMNG. The proteasomes were treated with Oprozomib at a concentration of $0.5 \mathrm{mM}$ at $25^{\circ} \mathrm{C}$ for 30 minutes. Proteasomes were loaded on linear $10-40 \%$ $(\mathrm{w} / \mathrm{v})$ sucrose gradients in purification buffer containing $7.5 \mathrm{mM}$ ATP, $5 \mathrm{mM}$ DTT, which were centrifuged at $220.000 \mathrm{x}$ g for 16 hours at $4{ }^{\circ} \mathrm{C} .400 \mu \mathrm{l}$ fractions were analyzed by SDS-PAGE, selected proteasome fractions were precipitated by the addition of $40 \%(\mathrm{v} / \mathrm{v})$ PEG400 and resuspended in purification buffer containing $7.5 \%(\mathrm{w} / \mathrm{v})$ sucrose, $7.5 \mathrm{mM}$ ATP, $5 \mathrm{mM}$ DTT and $0.01 \%$ (w/v) LMNG.

As a final step, proteasomes were fractionated on linear 10-45 \% (w/v) sucrose gradients in purification buffer containing $7.5 \mathrm{mM}$ ATP, $5 \mathrm{mM} \mathrm{DTT}$, which were centrifuged at $260.000 \mathrm{x}$ g for 16 hours at $4{ }^{\circ} \mathrm{C}$. Fractions containing 26/30S proteasomes were yet again identified by SDS-PAGE, precipitated by the addition of $40 \%(\mathrm{v} / \mathrm{v})$ PEG400 and resuspended in 2x purification buffer containing $15 \%$ (w/v) sucrose, $15 \mathrm{mM}$ ATP, $10 \mathrm{mM}$ DTT and $0.02 \%(\mathrm{w} / \mathrm{v})$ LMNG yielding the final purified protein preparation at $30 \mathrm{mg} / \mathrm{ml}$. Protein concentrations were determined by the Bradford assay (BioRad, Munich, Germany) using BSA as a standard.

This purification procedure reproducibly yields $45 \mathrm{mg}$ purified human 26/30S proteasomes, starting from $800 \mathrm{ml}$ S100 HeLa cytoplasmic extract at a concentration of $10 \mathrm{mg} / \mathrm{ml}$.

\subsubsection{Enzyme Kinetics}

To determine the chymotryptic-like activity of the $\beta 5$ catalytic active site of the human $20 \mathrm{~S}$ proteasome, kinetic measurements were performed using a FluoroMax-4 fluorescence spectrophotometer. Succinyl-Leucine-Leucine-Valine-Tyrosine-7-amido-4-methylcoumarin (Suc-LLVY-AMC) was utilized as substrate. Enzyme kinetics were determined by measuring the fluorescence emission of the hydrolyzed substrate at $460 \mathrm{~nm}\left(\lambda_{\text {ex }}=380 \mathrm{nM}\right)$ at a constant temperature of $37^{\circ} \mathrm{C}$.

The Suc-LLVY-AMC conversion was characterized using $50 \mathrm{nM}$ human 20S proteasomes. The proteasomes were pre-incubated in reaction buffer at $37^{\circ} \mathrm{C}$ for 3 minutes. The reaction was started by adding the substrate and the fluorescence signal was measured continuously.

When analyzing the first-order rate constant of inhibition, the reaction mixture containing the reaction buffer, $150 \mu \mathrm{M}$ Substrate and the respective inhibitor was pre-incubated. The following inhibitor concentration were used: $50 \mu \mathrm{m}$ Oprozomib, $50 \mu \mathrm{m}$ Dihydroeponemycin, $15 \mu \mathrm{m}$ Z-LLY-Ketoaldehyde. The reaction was started by the addition of human 
$20 \mathrm{~S}$ proteasomes at a final concentration of $50 \mathrm{nM}$. Suc-LLVY-AMC and the inhibitors were dissolved in DMSO and stored at $-80{ }^{\circ} \mathrm{C}$ until usage. Data analysis was carried out with OriginPro 9.1 (Origin Lab) and KaleidaGraph 4.03 (Synergy Software). To analyze the chymotryptic-like catalytic activity and catalytic activation of the $20 \mathrm{~S}$ proteasome the following equation was used:

$$
F(t)=F_{0}+\Delta F_{s s} \cdot t-\frac{\left(\Delta F_{s s}-\Delta F_{0}\right)}{k_{a c t}} \cdot\left(1-\exp ^{\left(-k_{a c t} \cdot t\right)}\right) .
$$

where $F_{0}$ denotes the fluorescence at time $t=0$ and $\Delta F_{s s}$ the increase in fluorescence (activity) in steady state after activation. $\Delta F_{0}$ denotes the increase in fluorescence (activity) at time $t=0$ prior to activation. $k_{a c t}$ denotes the first-order activation rate constant. The first-order inactivation rate constants were determined by using the equation with two exponential terms (Z-LLY-Ketoaldehyde) or two exponential terms and a linear term for the epoxyketone inhibitors Oprozomib and Dihydroeponemycin. The first term makes up the catalytic activation, whereas the second exponential term accounts for the catalytic inactivation by inhibition. The linear term signifies the residual activity of the proteasome after inactivation by epoxyketone inhibitors.

Data analysis was performed by Prof. Dr. Kai Tittmann, University Göttingen. The method description is according to Schrader at al., $2016^{120}$.

\subsubsection{Electronic Structure Calculations}

Cluster calculations were carried out on selected model systems, considering the Dihydroeponemycin, Oprozomib and Z-LLY-Ketoaldehyde inhibitors. The starting structures were based on the crystallographic data. All stationary points and constrained optimizations were carried out at the B3LYP-D3(BJ)/def2-SVP level of theory ${ }^{121,122,123,124}$, which include dispersion corrections as suggested by Grimme, with a Becke-Johnson type damp-

ing ${ }^{125}$. The nature of the stationary points was confirmed by frequency calculations. The reaction energy profiles are provided at the level of theory used for the optimizations (B3LYP-D3/def2-SVP).

Free-energy corrections were computed from frequency analysis under the rigid-rotor harmonic approximation of the latter structures. The electronic energy was determined at the B3LYP-D3/def2-TZVP level of theory, under the COSMO continuum solvation model ${ }^{126}$. Diethyl ether was employed as the solvent $(\varepsilon=4.3)$, which is commonly chosen to model protein-like environments ${ }^{127}$. All free-energy values reported correspond to the sum of the latter electronic energies and the thermodynamic corrections computed with the smaller def2-SVP basis set. All calculations were carried out with the Orca 3.0.3 program pack- 
$\operatorname{age}^{128}$.

Cluster quantum chemical calculations were performed by Prof. Dr. Ricardo Mata, University Göttingen. The method description is according to Schrader at al., $2016^{120}$.

\subsubsection{Crystallization Methods}

\subsubsection{Preparation of Protein Crystals}

The basis for all protein crystallizations experiments is formed by the phenomenon that a protein will stay in solution only upto a certain concentration, depending on its solubility limit. Crystallization requires bringing the macromolecule to a supersaturation state. Introducing the sample to a precipitation agent can promote the nucleation of protein crystals in the solution.

The two main techniques to obtain crystals are vapor diffusion and batch crystallization. Vapor diffusion is carried out in a hanging or sitting drop set up: A drop containing a mixture of protein and precipitant is sealed in an environment of pure precipitant (Figure 2.1, A). Over time, dehydration of the protein drop takes place until an equilibrium between drop and precipitant is formed. During the vapour diffusion method, a protein solution will start to concentrate from an undersaturated state to a supersaturated state. If crystals are formed the concentration of the free protein in the drop will decrease and crystals grow until the concentration of protein drops below to, or below the solubility line (Figure 2.1, B). The vapor diffusion techniques are easily adjustable by increase or decrease of the starting protein concentration or by the ratio of protein to precipitant or even by ratio of protein-precipitant mixture to the precipitant reservoir.

In the batch approach, the protein is mixed with a defined amount of the precipitant. To prevent the drop from drying out, the procedure is performed under a paraffin/mineral oil film, as shown in Figure 2.1, A. If the concentration of the precipitant is chosen in such a way that the solution is in the metastable zone, a successful crystallization might occur (Figure 2.1, B).

For the human $20 \mathrm{~S}$ proteasome, crystallization attempts were carried out by the vapor diffusion method. With a vast number of possible crystallization conditions it took time until different precipitant solutions and effects of $\mathrm{pH}$, temperature and additives were analyzed and a final crystallization condition was found. The new crystal form of the human 20S proteasome was obtained using the precipitant PEG3350 in combination with the buffer substance BisTris at pH 6.5 and the salt additive $\mathrm{MgCl}_{2}$ (Crystallization buffer). 
A

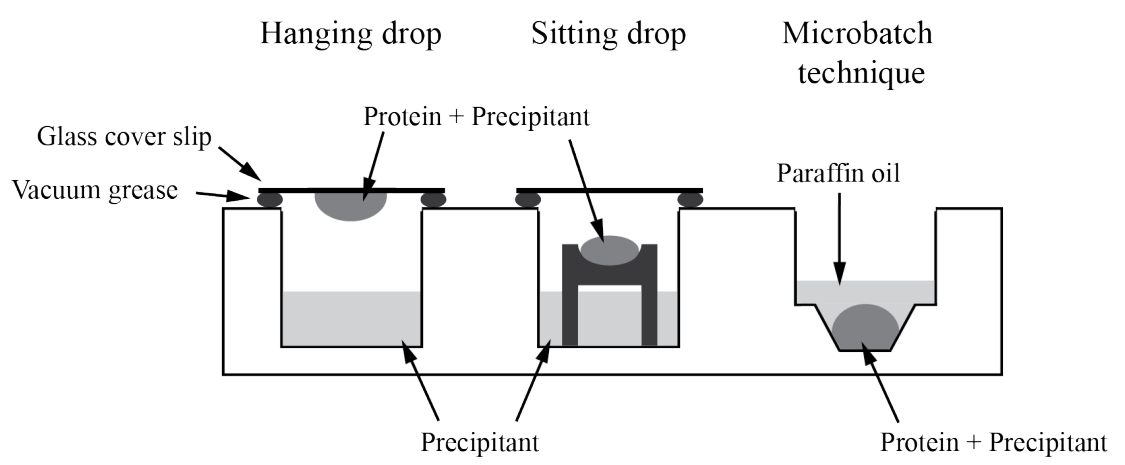

B

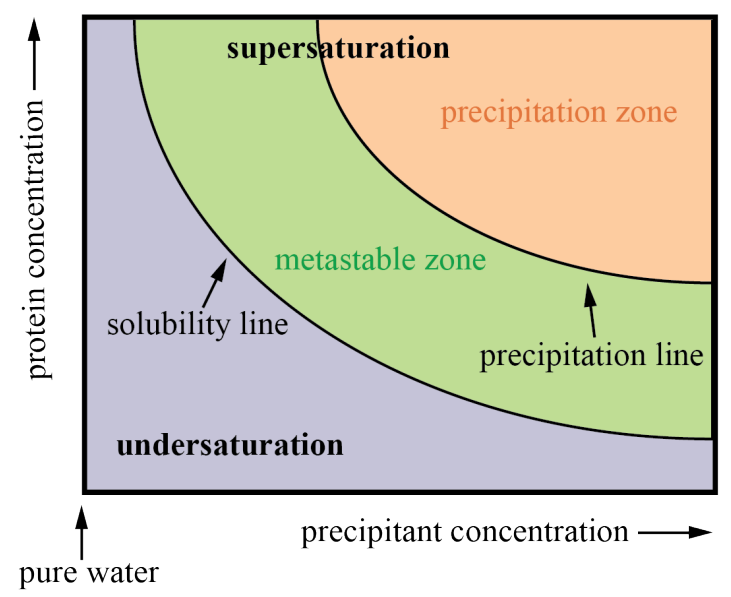

Figure 2.1: Crystallization techniques and the crystallization phase diagram. (A) Schematic representation of vapor diffusion techniques hanging and sitting drop and batch crystallization technique. (B) Schematic representation of a two-dimensional phase diagram, illustrating the change of protein concentration against precipitating agent concentration. Two areas are separated by the solubility line: the undersaturated and the supersaturated state of a proteins solution. The supersaturated area comprises the metastable and the precipitation zones, whereby crystals will eventually appear in the metastable phase and no crystals are formed in the precipitation zone. Modified from Bernhard Rupp ${ }^{129}$.

\subsubsection{Postcrystallization Treatment}

To improve the diffraction quality of macromolecular complexes it is almost always required to perform a postcrystallization treatment after crystals are obtained. Initial crystals are often of poor quality and unsuitable for X-ray studies. Postcrystallization is a method that provides the possibility to increase the diffraction quality of crystals. For the human 20S proteasomes one crucial step in crystal preparation is the dehydration, resulting in a controlled reduction of solvent molecules in the crystal and decrease of volume of the crystal by altering the crystal packing. These rearrangements promote new crystal contacts and thereby result in better diffracting crystals. Dehydration is performed by an increase or an introduction of precipitant: here PEG3350 and MPD. 


\subsubsection{X-ray Analysis Methods}

\subsubsection{Crystal Mounting and Diffraction Data Collection}

After post crystallization treatments the human $20 \mathrm{~S}$ proteasome crystals are ready for harvest. Crystals are harvested in Litholoops mounted on a standard $18 \mathrm{~mm}$ stainless steel pin and plunged in liquid nitrogen or directly transfered to a nitrogen gas stream at $100 \mathrm{~K}$. X-ray diffraction data was collected under cryogenic conditions at $100 \mathrm{~K}$. Initial crystal screening and data collections were performed at beamline ID23-I, ESRF, Grenoble, France using the PILATUS 6M detector. The high-resolution diffraction datasets were collected at the P14 beamline of the Petra III storage ring, EMBL, Hamburg, using the MD3 diffractometer with the spindle axis mounted in a vertical and downward configuration and the PILATUS 6M detector.

At the P14 beamline, a smooth "top-hat" X-ray beam profile with a total photon flux of up to $2 \times 10^{13} \mathrm{ph} / \mathrm{sec}$ was created by the installation of focusing Compound Refractive Lenses $20.6 \mathrm{~m}$ upstream of the sample position and slits $0.3 \mathrm{~m}$ upstream. To get beam-sizes matching the crystal sizes the slit opening was adjusted $\left(150 \times 200 \mathrm{\mu m}^{2}\right.$ in linear dimensions). Exposing the whole crystal provided by far the best resulting datasets. Standard setups with small X-ray beams or helical data collection only resulted in $2.3 \AA$ maximal resolution.

Crystals were exposed $40 \mathrm{~ms}$ per image with a gamma-slicing of $0.05-0.1$ in a total rotation range of $180^{\circ}-360^{\circ}$ at $50 \%$ attenuation. The collected datasets were rapidly scaled and integrated with the XDS program package.

\subsubsection{Structure Determination}

For model building the initial phases were determined by molecular replacement using the murine 20S structure (PDB ID: 3UNE). Model building and optimization was performed using COOT ${ }^{111}$. Subsequent refinement was performed using the program REFMAC5 in the CCP4 package ${ }^{130}$. Final graphic representations of details in the crystal structure were completed using the programs PYMOL and COOT. For modeling the bound inhibitors, complete stereo-chemical descriptions of double- or single-covalent link attachments were created using the program JLigand ${ }^{114}$ and applied in real-space fitting with COOT followed by a refinement with REFMAC5.

\subsubsection{Identification of Ions}

The identification of many ions in the native $20 \mathrm{~S}$ structure was validated by anomalous difference Fourier maps. For ligands in question, soaking experiments with anomalous 
scatterers were performed: $\mathrm{Cl}^{-}$ions were identified by their anomalous Fourier differences at $6.5 \mathrm{keV}$ and by exchange against $\mathrm{Br}^{-}$ions and measurements at the bromide K- absorption edge $(13.47 \mathrm{keV}) . \mathrm{Mg}^{2+}$ ions were identified by exchange through soaking crystals with $\mathrm{Mn}^{2+}$ ions and measurements at the manganese absorption edge $(6.5 \mathrm{keV}) . \mathrm{K}^{+}$ions were identified by their residual anomalous scattering at $6.5 \mathrm{keV}$, non-exchangeability by either $\mathrm{Br}^{-}$or $\mathrm{Mn}^{2+}$ and by measuring the coordination distances to neighboring residues within the structure.

\subsubsection{Sample Preparation for Electron Microscopy}

\subsubsection{GraFix}

The purified human 26S proteasomes were prepared for EM analysis by a fixation protocol: First, the sample was fixated in batch, using $2 \mathrm{mM}$ of the crosslinking agent BS3 for 30 minutes at $4{ }^{\circ} \mathrm{C}$. The BS3-fixed protein sample was then loaded onto 10-30 \% (w/v) GraFix gradient in which the complexes are centrifuged into an increasing concentration of the fixation reagent glutaraldehyde. The gradients contained 0-0.05\% (w/v) glutaraldehyde and were centrifuged for 16 hours at $114000 \mathrm{x}$ g. The human $26 / 30 \mathrm{~S}$ proteasomes sediment to $2 / 3$ of the gradient and the exact fraction is determined by fractionation and dotblot analysis. Dotblot is performed by pipetting $2 \mu \mathrm{l}$ of each sample fraction onto a nitrocellulose membrane and subsequent staining with amido black solution. After fractionation, the sample is immediately quenched by adding $20 \mathrm{mM}$ of sodium aspartate (pH 6.5).

\subsubsection{Negative Staining}

The fractions containing the $26 / 30 \mathrm{~S}$ proteasomes were analyzed by negative stain electron microscopy. A $2 \%(\mathrm{w} / \mathrm{v})$ uranyl formate solution was prepared freshly in double distilled water. In-house prepared carbon film was floated on the protein solution for up to 1 minute, depending on the protein concentration. The foil with the attached protein complexes was picked up by a holey carrier carbon film on a copper grid. Excess liquid was removed by gentle blotting of the grid with a filter paper. The grid containing foil and particles was then transfered onto the staining solution for 1 minute. The excess staining solution was blotted with a filter paper and the grid was allowed to air dry at room temperature for at least 10 minutes. 


\subsubsection{Vitrification}

After determination of the sample's quality by negative stain electron microscopy the sample was prepared for subsequent vitrification for cryo-EM analysis. For a successful cryo grid preparation the sucrose had to be removed from the protein sample. Almost all sucrose is removed by buffer exchange on Zeba Spin desalting columns and the particles were absorbed by continuous foil, as described before. The foil was picked up by a quantifoil grid containing a thick carbon film. Thereafter, the grid is loaded into the Vitrobot or Leica EM GP apparatus. $4 \mathrm{\mu l}$ of water is loaded to one side of the grid before blotting at $4{ }^{\circ} \mathrm{C}$ at $100-75 \%$ humidity. The grid was then plunge-frozen into liquid ethane and the thin water film with the particles on the grid was vitrified. Grids were stored in liquid nitrogen until usage.

\subsubsection{Imaging of Electron Microscopic Data}

Negative stain electron microscopic analysis was performed on a CM200 FEG microscope. Images were taken at $1 \mu \mathrm{m}$ defocus on a twice binned CCD camera at a magnification of $88000 \mathrm{x}$. The corresponding pixel size is $2.5 \AA$ /pixel. High-resolution cryogenic electron microscopy was performed on a Titan Krios TEM microscope equipped with a $\mathrm{C}_{\mathrm{S}}$-corrector. 17 image frames were recorded on a direct electron detector (Falcon2) with a dosage of 50 electrons per $\AA^{2}$ with a pixel size of $1.27 \AA$.

\subsubsection{Image Processing}

Image processing of the human 26S proteasome was performed by Dr. David Haselbach, Structural Dynamics Department at the Max Planck Institute for Biophysical Chemistry, Göttingen.

\subsubsection{Micrograph Analysis and Particle Picking}

Individual image frames were aligned and weighted according to electron dose to reduce the effects of drift and charging using the software unblur ${ }^{131}$. The Contrast Transfer Function (CTF) was determined on averaged micrographs with Gctf ${ }^{112}$. Particles were selected with the software Gautomatch ${ }^{132}$ and individual particle coordinates were refined by alignment against a projected model of the $26 \mathrm{~S}$ proteasome. Particles were cropped in a window 1.5 times in diameter. 


\subsubsection{2D Processing}

For $2 \mathrm{D}$ analysis, particles were down-sampled to a pixel size of 4 to $8 \AA$ /pixel as well as filtered and normalized. Several image sorting steps to remove contaminations, blurred images or broken particles were performed: First, power spectra for each particle were calculated and classified using a hierarchical cluster scheme. The resulting class averages were visually inspected for the Thon ring quality. Strongly charged and/or blurred classes were discarded. Second, iterative rounds of multi-reference alignment and 2D classification were performed. Particles sampled into classes which do not show intact proteasome particles were discarded.

\subsubsection{Initial Model Building}

After particle sorting, the best class averages were chosen and an initial 3D model was generated with the program simple PRIME ${ }^{116}$. This independent approach produces 3D models based on an iterative projection-matching approach without the need of an initial model.

\subsubsection{Conformational Sorting}

The resulting 3D model was used as the initial reference for 3D classification, using the program RELION ${ }^{115}$. Here, all particles were classified according to the two main conformational states (non-rotated and rotated). The flexible protein Rpn1 was masked out because it interferes with the alignment.

\subsubsection{Refinement and Resolution Determination}

The particles belonging to the non-rotated state were refined using the "auto-refine" routine in RELION. Further hierarchical sorting was performed in RELION to sort particles regarding to various regulatory particle conformations: A series of $3 \mathrm{D}$ classification steps without alignment with decreasing mask size was performed. After 4 refinement steps, 98000 particles contributed to the final 26S proteasome model (details in Results section). A local resolution map was estimated in ResMap ${ }^{133}$, calculating local Fourier Shell Correlation (FSC) values in a sphere with a diameter of 13 voxels, scanning the entire 3D model. 


\subsubsection{Model Building}

For the 20S particle, the crystal structure of the Oprozomib-inhibited human 20S proteasome was used as initial atomic coordinate model (PDB entry: 5LEY) ${ }^{120}$. Models of each regulatory particle protein were generated with Robetta ${ }^{134,135}$ and docked as rigid bodies into the EM density map with UCSF Chimera ${ }^{117}$. Nucleotides were placed by fitting the crystal structure of PAN (PDB entry: $3 \mathrm{H} 4 \mathrm{M}$ ) ${ }^{136}$ into the density.

Secondary structure prediction for regions that were modeled partly or completely de novo were obtained using the server psipred ${ }^{137}$. The initial rigid body refinement was performed using real space refinement in Phenix ${ }^{138}$ and modeling was carried out manually in COOT.

Secondary structure restraints were generated using phenix.ksdssp and all secondary structure restraints were visually inspected and required restraints were added manually. The real space refinement was performed over several iterative rounds.

\subsubsection{Validation}

For datasets which yield two or more main conformations, the structural model is validated by a cross reference test. Here, the particles corresponding to one conformation were refined on a model with the alternative conformation. The final 3D reconstruction relaxed back to the originating model and the sorting was considered as valid.

\subsubsection{Visualization of the Conformational Space of the Human 26S Proteasome}

If not stated differently, particles with and without Oprozomib bound to the proteasome were treated as separate subsets. All particles of one subset were refined against the initial 26S proteasome map using RELION to gain alignment parameters. This reduces the subsequent calculations in the classification step. The resulting aligned images were randomly split in subsets of 100,000 particles and a RELION 3D-classification ${ }^{139}$, yielding 40 classes per subset, was performed, without aligning the particles again. Each resulting $3 \mathrm{D}$ class was further refined with the assigned particles using RELION.

In total, 346 classes were obtained. To further analyze the motions between the classes we used principle component analysis (PCA). The result of a PCA are eigenvectors that describe the largest covariance within the dataset. In aligned 3D volumes of the same

molecule, the largest covariance are primarily movements within the molecule ${ }^{140}$. The eigenvectors can be used as conformational coordinates ${ }^{141}$. Before applying PCA, the refined 3D-classes were aligned in UCSF Chimera against a model of the most rigid part of 
the $26 \mathrm{~S}$ complex; the $20 \mathrm{~S}$ proteasome subcomplex. This is necessary to avoid calculating eigenvectors which describe shifts and rotations of the 3D-classes among themselves. 3D classes from datasets with and without Oprozomib were combined and normalized, and subsequently eigenvectors were calculated using PCA. Hence, the eigenvectors describe the movements found in both datasets and allow us to compare the results. One can then describe the conformers $X_{i}$ on a coordinate $e_{n}$ (the eigenvector describing movement $n$ ) by:

$$
X_{i}=a_{n, i} e_{n}+\bar{X}
$$

where $\bar{X}$ is the average volume, $e_{n}$ is a specific eigenvector and $a_{n, i}$ is a linear factor. In other words, $e_{n}$ is a conformational coordinate and $a_{n, i}$ places the conformer $X_{i}$ at its specific place on this conformational coordinate. The addition of the mean volume results from the definition of PCA. Eigenvectors and their corresponding trajectories can be sorted according to their contribution to the overall mobility.

The movements described by the conformational coordinates might however be composed out of several primitive motions of the molecule. Therefore, one cannot assign a single measure (e.g. rotation angle, translation movement) to all of these movements. The following movements were found and are listed descending in the value of the covariance:

Table 2.5: Eigenvectors and the corresponding conformational changes.

\begin{tabular}{c|l}
\hline Eigenvector & Description \\
\hline 1 & Rotation of the Lid and translation of the ATPase domain \\
2 & Resolution differences \\
3 & Conformational stabilization of Rpn9 \\
4 & Conformational stabilization of Rpn5, 10 and 12; Movement of Rpn1 \\
5 & Motion of Rpn1; Conformational stabilization of Rpn9, 10 \\
6 & Translation of Rpn2, 3, 7, 12 \\
7 & Stretching of the Lid; Movement of the central helical bundle \\
8 & Rpn3, 6, 8, 11); Movement of ATPase domain \\
9 & Rise of the ATPase (including Rpn1); Sinking of Rpn2 and 8 \\
10 & Small movements \\
\hline
\end{tabular}

To understand the motion described by the eigenvectors, video trajectories where interpolated using equation 2.2 and subsequent morphing in UCSF Chimera. Then, for each $3 \mathrm{D}$ class the linear factors with respect to each eigenvector were determined. By placing the different conformers on the reaction coordinates energetic conclusions can be drawn. Knowing the number of particles assigned to each class, we can calculate their free energy 
differences $\Delta \Delta G$ by the BOLZMANN Factor:

$$
\Delta \Delta G=k_{B} T \ln \left(\frac{p_{i}}{p_{0}}\right)
$$

where $T$ is the absolute temperature, $p_{i}$ is the number of particles in state $i$ and $p_{0}$ is the number of particles in the most populated state ${ }^{142}$. The number of particles belonging to each 3D was counted from the respective data output files from RELION ${ }^{115}$. Free energy differences were calculated using equation 2.3 as multiples of $k_{B} T$. From equation 2.3 it becomes clear that regions with high energies have a lower number of single particles belonging to them. To visualize the energy landscape with and without Oprozomib, combinations of two eigenvectors were used as the axis of a new three-dimensional coordinate system. The 3D classes were placed as data points in these landscapes with the dimensions being the respective linear factors $a_{n, i}$ and $a_{m, i}$, and the difference in free energy $\Delta \Delta G$. We could then describe energy landscapes in which the molecule moves by interpolating between these discrete states.

To account for false-positives, for example 3Ds which are classified in two separate classes but do only differ slightly, we applied a final binning of close data points. Therefore, all data points within a given distance were averaged. This distance was set to the halfwidth of the peak around one linear factor. These landscapes have important limitations. In contrast to for example Molecular Dynamics simulations, the 3D volumes yielded from single particle analysis sample the conformational space discrete and sparse. Hence, large areas in the landscapes, especially those of high energy, are highly interpolated. This is also apparent in the fact that the very low sampled areas close to unfolding and complex decomposition are not accessible to this method. However, this method offers an opportunity to quantify the results of 3D classifications and, as in our specific case, allows us to learn about the influence of small molecule binding on the functional conformational landscape of a macromolecular machine.

This method was developed by Dr. David Haselbach and Felix Lambrecht in the Structural Dynamics Department at the Max Planck Institute for Biophysical Chemistry, Göttingen. 


\section{Chapter 3}

\section{Results}

\subsection{Native Human 20S Proteasomes and in Complex with Inhibitors}

The structure determination of macromolecular complexes by X-ray crystallography depends on the availability of crystals that diffract to suitably high resolution. Crystallization conditions are known for $20 \mathrm{~S}$ proteasomes from various organisms, and crystal structures up to a resolution of $2.4 \AA$ were previously published ${ }^{16,23,25,143}$.

In this section, the outcome from novel purification strategies, new crystallization conditions and post-crystallization procedures with the resulting high-resolution structures of the native human $20 \mathrm{~S}$ proteasome and its complexes with $20 \mathrm{~S}$ inhibitors are presented.

\subsubsection{Purification and Crystallization of the Human 20S Pro- teasome}

The starting point for the successful structure determination of human 20S proteasomes at a significantly higher resolution is a novel concept of protein purification. For human $20 \mathrm{~S}$ proteasomes, a chromatography-free purification technique was established and optimized (Figure 3.1, A for a schematic description of the procedure and Figure 3.1, B for a representative SDS-PAGE of individual steps of the 20S proteasome purification).

The purification strategy is simple, rapid, and enables the purification of human 20S proteasomes within 48 hours. The purification is chromatography-free and starts with the treatment of cytosolic extract of HeLa cells. After centrifugation of the extract at $30000 \mathrm{x} \mathrm{g}$ for 30 minutes (S30 extract preparation), the supernatant is incubated with 2-Iodoacetamide and N-Ethylmaleimide for 30 minutes and centrifuged at $100000 \mathrm{x} \mathrm{g}$ for 2 hours (S100 extract preparation). The resulting supernatant is then subjected to a combination of differential precipitation with the polymer PEG400 and sucrose gradient centrifugation. Fractions containing 20S proteasomes are identified by SDS-PAGE after 
each purification step. This procedure reproducibly yields $20 \mathrm{mg}$ purified human $20 \mathrm{~S}$ proteasomes, starting from $300 \mathrm{ml}$ cytosolic HeLa extract at $10 \mathrm{mg} / \mathrm{ml}$.

The resulting highly soluble and stable human 20S proteasomes have a specific activity of $13 \mathrm{nmol} / \mathrm{mg}^{-1} / \mathrm{min}^{-1}$ in the activation phase (pre-steady-state) and $60 \mathrm{nmol} / \mathrm{mg}^{-1} / \mathrm{min}^{-1}$ in the steady-state phase (Figure 3.1, C). This means that the human $20 \mathrm{~S}$ proteasomes purified by the chromatography-free purification method have a higher specific activity by a factor of 10 than reported for previous $20 \mathrm{~S}$ proteasome preparations ${ }^{22}$.

A

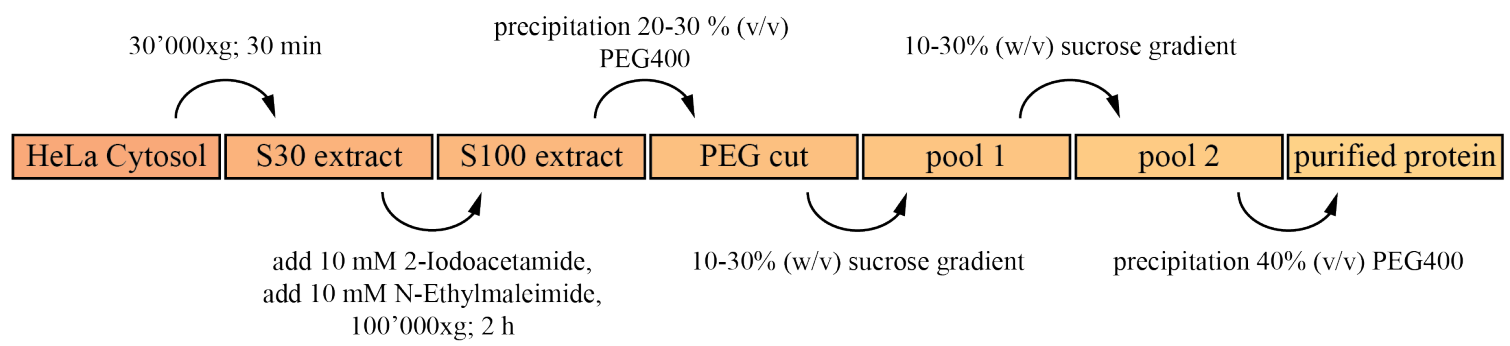

B

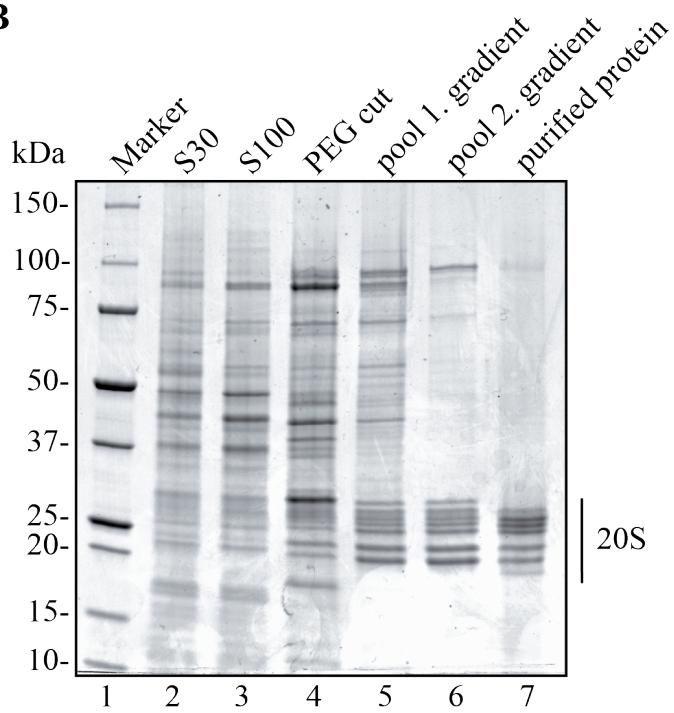

C

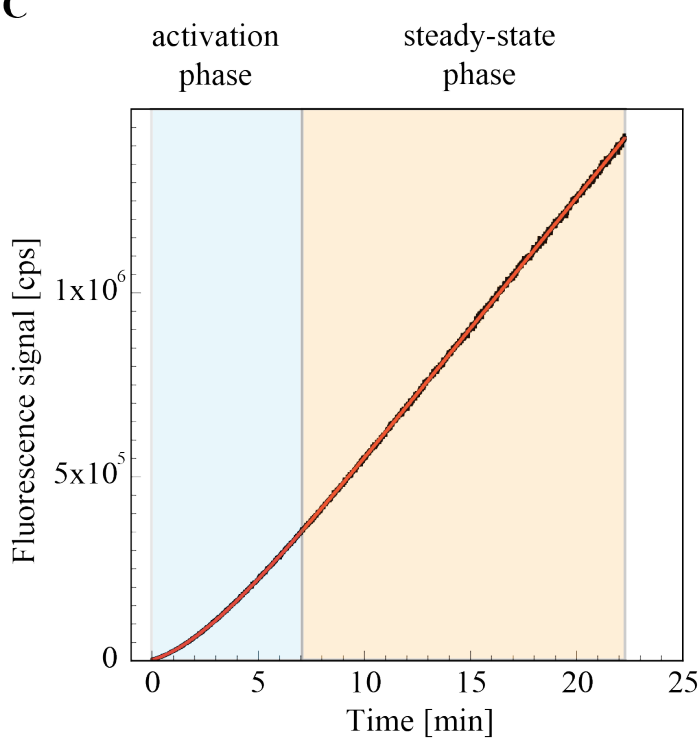

Figure 3.1: The high-throughput purification procedure. (A) Schematic representation of the entire high-throughput pipeline to purify human $20 \mathrm{~S}$ proteasomes from HeLa cytoplasm. (B) SDSpolyacrylamide gel electrophoresis analysis of distinct steps of the purification procedure. Samples of the S30 (lane 2) and S100 (lane3) extract, the 20-30 \% (v/v) PEG400 cut (lane 4), the pool of the first (lane 5) and second (lane 6) sucrose gradient centrifugation, the purified 20S proteasome sample (lane 7) and a marker as reference (lane 1) are depicted. (C) Activity of the human 20S proteasome from the chromatography-free purification. The increase in fluorescence signal of AMC release by proteolytic cleavage is plotted against time. Figure adapted from Schrader et al., $2016^{120}$. 


\subsubsection{Preparation of Human 20S Proteasome Crystals}

The freshly purified protein is diluted with purification buffer to a concentration of $7.5 \mathrm{mg} / \mathrm{ml}$ and crystallized by mixing $0.5 \mu \mathrm{l}$ protein and $0.5 \mu \mathrm{l}$ crystallization buffer in Chryschem sitting drop vapor diffusion plates over a $500 \mu \mathrm{l}$ reservoir of crystallization buffer. Within 4 hours, nucleation takes place and the first small crystals are detectable. To depict the time course of human 20S crystallization, a time-lapse experiment was performed and the results are shown in Figure 3.2. Within 24 hours the crystal grows to its full size, $150 \times 150 \times 200 \mathrm{\mu m}^{3}$.

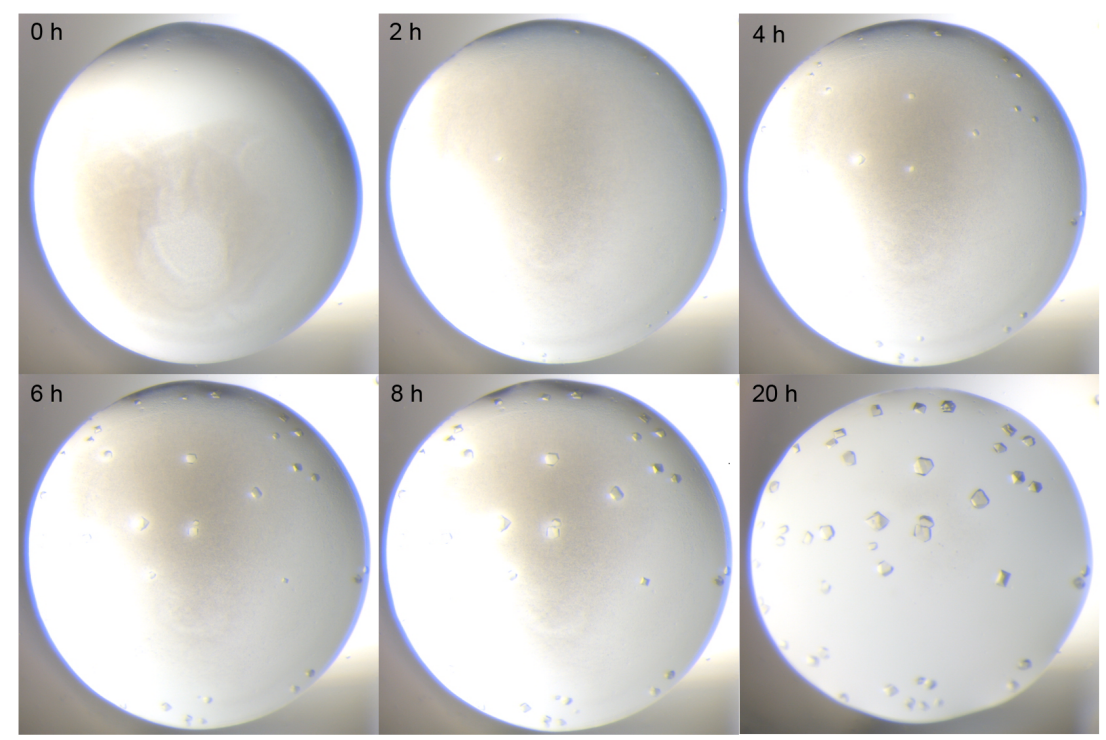

Figure 3.2: Time-lapse experiment of human 20S crystallogenesis. Timepoints are $0,2,4,6,8$, and 20 hours. Nucleation takes place after 4 hours and crystals grow within 24 hours to their full size. Figure adapted from Schrader et al., $2016^{120}$.

\subsubsection{Postcrystallization Treatments}

To gain a high reproducibility in crystal diffraction to $1.8-2.2 \AA$ resolution, the human $20 \mathrm{~S}$ proteasome crystals need to be stabilized and dehydrated in a controlled manner. Extensive time and effort was spend to find the optimal procedure.

Finally, three steps seem to be critical and necessary for highly reproducible crystal diffraction: 1) Crystals initially grown at $18{ }^{\circ} \mathrm{C}$ are transfered to incubators where the temperature is decreased to $4{ }^{\circ} \mathrm{C}$ within 24 hours. When at $4{ }^{\circ} \mathrm{C}$ the crystals are carefully transported into the $4{ }^{\circ} \mathrm{C}$ room. All postcrystallization treatments take place at $4{ }^{\circ} \mathrm{C}$. 2) The seals are removed and $1 \mu \mathrm{l}$ of the reservoir solution is added to the $1 \mu \mathrm{l}$ drop. Subsequently, $2 \mu$ of the crystal stabilization buffer is added to the drop and the reservoir solution is exchanged against the stabilization buffer. The wells are re-sealed and equilibration against the new reservoir by vapor-diffusion takes place in excess of 8 hours at $4{ }^{\circ} \mathrm{C}$. 3) If needed, ligands (e.g. proteasome inhibitors) are soaked into the crystal when 
analyzing the inhibitor-proteasome complexes. Inhibitors are soaked into the crystals by adding $4 \mathrm{\mu l}$ of dehydration buffer, which additionally contains $5 \mathrm{mM}$ of the proteasome inhibitor to gain a final concentration of $2.5 \mathrm{mM}$. The wells are re-sealed and equilibration against the new reservoir by vapor-diffusion takes place in excess of 12 hours at $4{ }^{\circ} \mathrm{C}$. The full post crystallization protocol is concluded within 30 hours. 


\subsubsection{Native Crystal Structure of the Human 20S Proteasome}

In this thesis, a high resolution structure of the native $20 \mathrm{~S}$ proteasome at an unprecedented resolution of $1.8 \AA$ is presented. A diffraction pattern of the stabilized and dehydrated human 20S proteasome crystals measured at beamline P14 in Hamburg is shown in Figure 3.3, A.

The determination of the native structure at $1.8 \AA$ provides better insights into the architecture of the entire particle and its active sites. The obtained structures display excellent stereochemistry with values for $\mathrm{R}_{\text {work }}=18 \%$ and $\mathrm{R}_{\text {free }}=21 \%$ (Table 3.1 ) and the final crystallographic maps reveales clear electron densities for all 6724 residues and allows the identification of several ligands present in buffers used for purification and crystallization (Figure 3.3, B). The identity of many ions was validated by anomalous difference Fourier maps. In the final structure, 58 chloride, 15 magnesium and 6 potassium ions, in addition to several PEG molecules and more than 3500 localized water molecules were assigned.

Table 3.1: Data collection and refinement statistics of the native $20 \mathrm{~S}$ crystals. Values in parenthesis denote values for the highest resolution shell. Number of unique reflections used in refinement are shown.

\begin{tabular}{|c|c|c|}
\hline Tag & Native & Native \\
\hline PDB ID & 5LE5 & 5LEX \\
\hline Divalent salt & $\mathrm{MgCl}_{2}$ & $\mathrm{Mg}(\mathrm{OAc})_{2}$ \\
\hline Space group & \multirow{2}{*}{\multicolumn{2}{|c|}{$\mathrm{P} 2_{1} 2_{1} 2_{1}$}} \\
\hline Unit cell & & \\
\hline a $(\AA)$ & 113.4 & 113.9 \\
\hline $\mathrm{b}(\AA)$ & 202.8 & 203.1 \\
\hline c $(\AA)$ & 316.0 & 316.0 \\
\hline \multicolumn{3}{|l|}{ Data collection } \\
\hline Wavelength $(\AA)$ & \multicolumn{2}{|c|}{0.976} \\
\hline Resolution range & $107-1.80$ & $50-2.20$ \\
\hline$(\AA)$ & $(1.90-1.80)$ & $(2.28-2.20)$ \\
\hline No. of reflections & 8923661 & 2519654 \\
\hline $\begin{array}{l}\text { No. of unique } \\
\text { reflections }\end{array}$ & 633728 & 366080 \\
\hline Completeness (\%) & $99.9(99.7)$ & $99.2(98.1)$ \\
\hline$<\mathrm{I} / \operatorname{SigI}>$ & $18.5(0.91)$ & $13.0(0.9)$ \\
\hline $\mathrm{CC}_{1 / 2}$ & $1.00(0.32)$ & $1.00(0.36)$ \\
\hline $\mathrm{R}_{\mathrm{p} . \mathrm{i} . \mathrm{m}}$ & $0.026(0.71)$ & $0.039(0.87)$ \\
\hline \multicolumn{3}{|l|}{ Refinement } \\
\hline Rwork & 0.184 & 0.176 \\
\hline Rfree & 0.213 & 0.220 \\
\hline \multicolumn{3}{|l|}{ No. of atoms } \\
\hline Protein & 48205 & 48134 \\
\hline Ligand/Solvent & 3712 & 3493 \\
\hline $\begin{array}{l}\text { R.m.s.d. bond } \\
\text { length }(\AA)\end{array}$ & 0.018 & 0.015 \\
\hline $\begin{array}{l}\text { R.m.s.d. bond } \\
\text { angle }(\AA)\end{array}$ & 1.92 & 1.72 \\
\hline Ramachandran & & \\
\hline $\begin{array}{l}\% \text { most favored/ } \\
\text { /favored }\end{array}$ & $95.88 / 3.06$ & $95.60 / 3.29$ \\
\hline
\end{tabular}

In Figure 3.4 the active sites of all three catalytic sites of the $20 \mathrm{~S}$ proteasome and the identified ligands are shown. At $1.8 \AA$ resolution, a number of differences with respect to the previous published structures become visible. Particularly, a chloride ion is present 
A

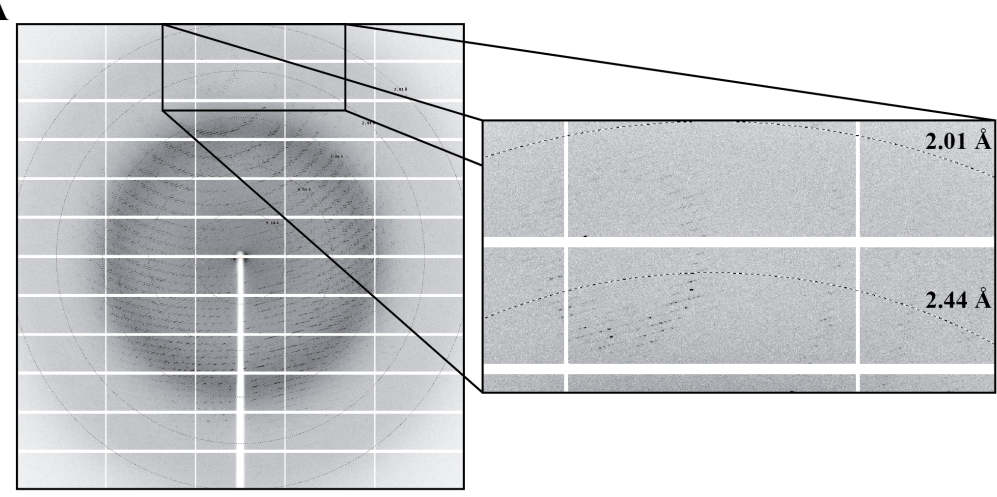

B

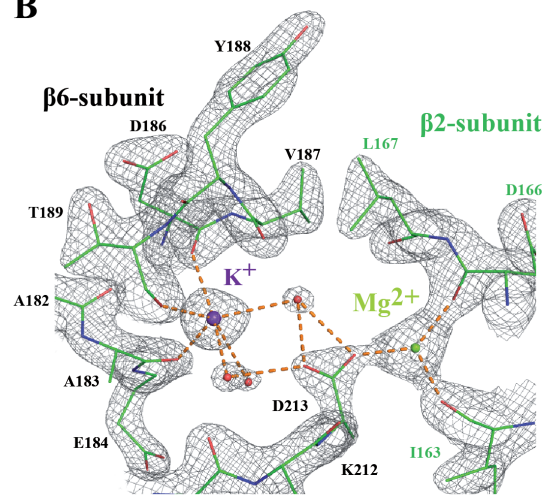

Figure 3.3: Diffraction pattern and structural details at $1.8 \AA$ resolution. (A) A typical diffraction pattern of stabilized and dehydrated human $20 \mathrm{~S}$ proteasome crystals measured by the oscillation method at beamline P14, EMBL Hamburg. Beam size matched crystal size, exposure time was $40 \mathrm{~ms}$ and the oscillation range $0.05^{\circ}$. The diffraction limit is $1.8 \AA$. (B) The electron density gives insight into the quality of the high resolution structure at $1.8 \AA$ resolution. Shown is a potassium (magenta)-magnesium (light green) double ion site, stabilizing the $\beta 6$ - $\beta 2$ interface. Protein main chains and side chains belonging to the $\beta 6$ subunit are labeled in black. Protein main chains and side chains belonging to the $\beta 2$ subunit are labeled in green. The $\sigma_{\mathrm{A}}$-weighted electron density map is shown contoured at $1.5 \sigma$. Figure adapted from Schrader et al., $2016^{120}$.

in all three sites, which was previously described as a catalytic water (NUK). Soaking experiments, where crystals grown in $\mathrm{MgCl}_{2}$ were soaked with $\mathrm{Mg}(\mathrm{OAc})_{2}$, replace the $\mathrm{Mg}^{2+}$ ion against a water molecule. Additionally, up to three localized water molecules $\left(\mathrm{H}_{2} \mathrm{O}-1,-2,-3\right)$ are present in the active sites. These findings imply a new interpretation of the inhibition mechanism: A water in the NUK position might act as a proton shuttle. The putative role of this water was analyzed at a later point (see below).

A

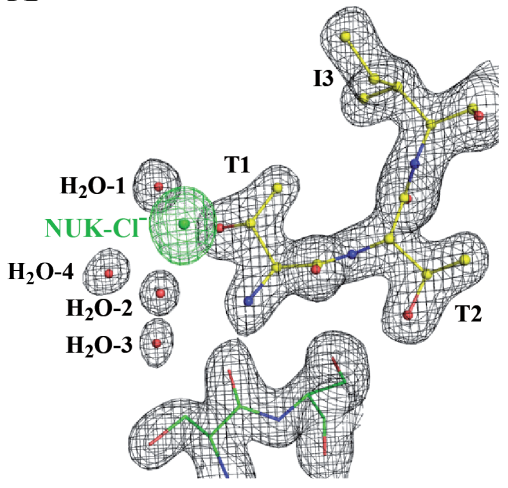

B1-subunit caspase-like active site
B

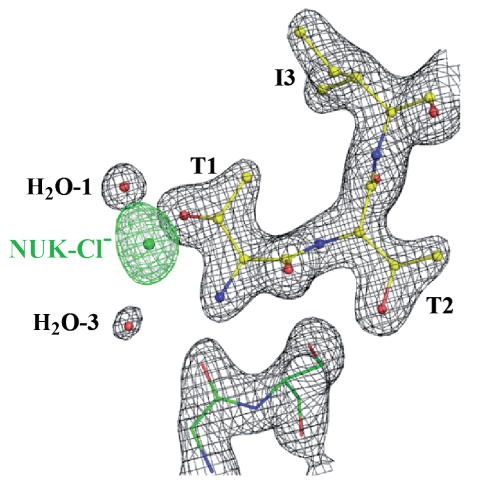

B2-subunit tryptic-like active site
C

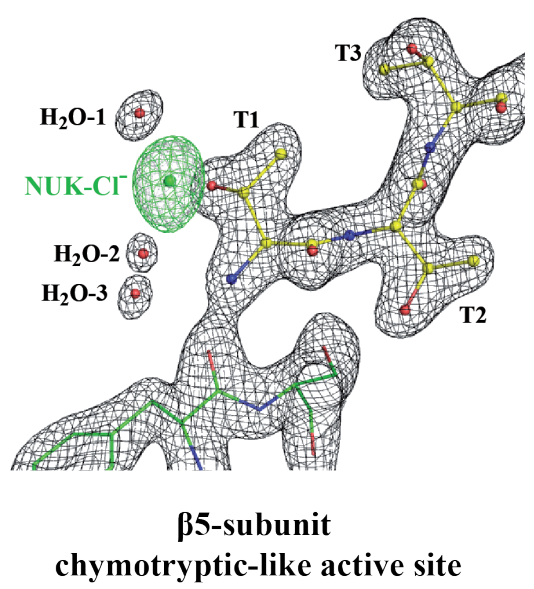

Figure 3.4: Close-up view of all three catalytic sites of the high resolution $20 \mathrm{~S}$ structure. (A) $\beta 1$-subunit caspase active site with Thr1, Thr2, Ile3, four $\mathrm{H}_{2} \mathrm{O}$ molecules and a NUK chloride (green). (B) $\beta 2$-subunit tryptic active site is shown in the same orientation as in (A). Shown are two $\mathrm{H}_{2} \mathrm{O}$ molecules, as well as the NUK chloride. (C) $\beta 5$-subunit chymotryptic active site depicted as in (A) and (B), shown are three $\mathrm{H}_{2} \mathrm{O}$ molecules and the NUK chloride. The $2 \mathrm{mFo}-\mathrm{DFc}$ electron density map is shown contoured at $1.5 \sigma$. Figure adapted from Schrader et al., $2016^{120}$. 


\subsubsection{Crystal Structures of Seven Inhibitor Complexes of the Human 20S Proteasome}

In addition to the native structures, six structures of the human $20 \mathrm{~S}$ proteasome in complex with inhibitors at resolutions between 1.9 and $2.4 \AA$ were elucidated. Four of these complexes describe proteasomes with inhibitors which are in clinical trials for the treatment of cancer. Binding of these inhibitors to the human 20S proteasome was structurally uncharacterized prior to this work. Oprozomib, Dihydroeponemycin, Ixazomib, and Delanzomib were chosen. They belong to three different inhibitor classes: $\alpha$ ', $\beta$ '-Epoxyketone, $\alpha$-Ketoaldehyde and Boronic acid. All inhibitors used in this experiments are described to have a specificity for the $\beta 5$ subunit and in all further steps the analysis of the inhibitors is restricted to this active site. In Table 3.2 the most important data collection details and refinement statistics are depicted.

Table 3.2: Crystallographic data collection and refinement statistics of inhibitor-20S complexes. Colors indicate the difference in inhibitor classes. Statistics for epoxyketone inhibitors (blue), ketoaldehyde inhibitors (violet) and boronic acid inhibitors (green) are listed, respectively the PDB entry ID. Values in parenthesis denote values for the highest resolution shell. Number of unique reflections used in refinement is shown.

\begin{tabular}{|c|c|c|c|c|c|c|c|c|}
\hline \multirow{2}{*}{$\begin{array}{l}\text { Inhibitor Class } \\
\text { Tag }\end{array}$} & \multicolumn{4}{|c|}{ Epoxyketone } & \multirow{2}{*}{$\begin{array}{l}\text { Ketoaldehyde } \\
\text { Z-LLY- } \\
\text { ketoaldehyde }\end{array}$} & \multicolumn{3}{|c|}{ Boronic acid } \\
\hline & Oprozomib & Oprozomib & Epoxomicin & $\begin{array}{l}\text { Dihydro- } \\
\text { eponemycin }\end{array}$ & & Bortezomib & Ixazomib & Delanzomib \\
\hline PDB ID & $5 \mathrm{LEY}$ & $5 \mathrm{LEZ}$ & 5LF0 & $5 \mathrm{LF} 1$ & $5 \mathrm{LF} 6$ & $5 \mathrm{LF} 3$ & 5LF7 & $5 \mathrm{LF} 4$ \\
\hline Divalent salt & $\mathrm{MgCl}_{2}$ & $\mathrm{Mg}(\mathrm{OAc})_{2}$ & $\mathrm{MgCl}_{2}$ & $\mathrm{MgCl}_{2}$ & $\mathrm{MgCl}_{2}$ & $\mathrm{MgCl}_{2}$ & $\mathrm{MgCl}_{2}$ & $\mathrm{MgCl}_{2}$ \\
\hline Space group & \multirow{2}{*}{\multicolumn{8}{|c|}{$\mathrm{P} 212121$}} \\
\hline Unit cell & & & & & & & & \\
\hline $\mathrm{a}(\AA)$ & 113.4 & 113.9 & 113.1 & 113.9 & 113.9 & 113.4 & 113.4 & 114.4 \\
\hline $\mathrm{b}(\AA)$ & 202.7 & 203.2 & 202.4 & 203.5 & 203.5 & 202.7 & 202.6 & 202.8 \\
\hline $\mathrm{c}(\AA)$ & 315.1 & 315.2 & 314.8 & 316.0 & 315.6 & 314.9 & 314.5 & 314.3 \\
\hline \multicolumn{9}{|l|}{ Data collection } \\
\hline $\begin{array}{l}\text { Resolution range } \\
(\AA)\end{array}$ & $\begin{array}{l}170-1.90 \\
(2.00-1.90)\end{array}$ & $\begin{array}{l}50-2.19 \\
(2.27-2.19)\end{array}$ & $\begin{array}{l}107-2.41 \\
(2.50-2.41)\end{array}$ & $\begin{array}{l}50-2.0 \\
(2.07-2.00)\end{array}$ & $\begin{array}{l}50-2.07 \\
(2.15-2.07)\end{array}$ & $\begin{array}{l}157-2.10 \\
(2.20-2.10)\end{array}$ & $\begin{array}{l}94-2.00 \\
(2.10-2.00)\end{array}$ & $\begin{array}{l}50-1.99 \\
(2.06-1.99)\end{array}$ \\
\hline No. of reflections & 2653718 & 2510786 & 2519995 & 4381423 & 2792074 & 2017022 & 3047303 & 4210019 \\
\hline $\begin{array}{l}\text { No. of unique } \\
\text { reflections }\end{array}$ & 526170 & 351230 & 262073 & 464668 & 403247 & 393306 & 459417 & 462522 \\
\hline Completeness (\%) & $97.5(99.4)$ & $99.3(94.3)$ & $99.9(99.6)$ & $99.8(98.3)$ & $96.4(72.3)$ & $98.7(89.1)$ & $99.8(99.6)$ & $99.1(90.0)$ \\
\hline$<\mathrm{I} / \mathrm{SigI}>$ & $15.3(0.83)$ & $15.2(1.1)$ & $13.1(0.83)$ & $12.2(1.0)$ & $12.9(0.6)$ & $12.4(0.9)$ & $14.6(1.0)$ & $11.3(1.0)$ \\
\hline $\mathrm{CC}_{1 / 2}$ & $1.00(0.24)$ & $1.00(0.40)$ & $1.00(0.28)$ & $1.00(0.39)$ & $0.99(0.22)$ & $1.00(0.26)$ & $1.00(0.29)$ & $0.99(0.34)$ \\
\hline Rp.im. & $0.041(1.03)$ & $0.036(0.81)$ & $0.060(1.06)$ & $0.038(0.69)$ & $0.043(1.0)$ & $0.052(0.87)$ & $0.041(1.1)$ & $0.045(0.81)$ \\
\hline \multicolumn{9}{|l|}{ Refinement } \\
\hline Rwork & 0.189 & 0.181 & 0.168 & 0.175 & 0.185 & 0.183 & 0.182 & 0.184 \\
\hline Rfree & 0.224 & 0.222 & 0.227 & 0.209 & 0.226 & 0.225 & 0.215 & 0.217 \\
\hline \multicolumn{9}{|l|}{ No. of. atoms } \\
\hline Protein & 48208 & 48372 & 48372 & 48302 & 48219 & 48331 & 48272 & 48272 \\
\hline Ligand/solvent & 3756 & 3882 & 3385 & 3839 & 3718 & 3888 & 3809 & 3794 \\
\hline $\begin{array}{l}\text { R.m.s.d. bond } \\
\text { length }(\AA)\end{array}$ & 0.016 & 0.015 & 0.018 & 0.014 & 0.013 & 0.014 & 0.013 & 0.012 \\
\hline $\begin{array}{l}\text { R.m.s.d. bond } \\
\text { angles }(\AA)\end{array}$ & 1.80 & 1.75 & 1.89 & 1.71 & 1.65 & 1.72 & 1.68 & 1.642 \\
\hline $\begin{array}{l}\text { Ramachandran } \\
\% \text { most favoured } \\
\text { /favoured }\end{array}$ & $95.58 / 3.30$ & $95.24 / 3.45$ & $95.91 / 2.88$ & $95.68 / 3.16$ & $95.49 / 3.35$ & $95.67 / 3.12$ & $95.62 / 3.24$ & $95.88 / 2.99$ \\
\hline
\end{tabular}

The high-resolution human 20S proteasome structures in complex with inhibitors reveal differences to earlier published data. The most prominent differences are visible in the 20S-Oprozomib structure at $1.9 \AA$ resolution. After refinement, the linkage of Oprozomib 
to the active site revealed a cyclic moiety. Unlike the previously predicted 1,4-morpholine ring closure, the electron density map revealed a larger, 7-membered ring structure. This ring-shaped structure could be modeled as a 1,4-oxazepane linkage, where the C6-methyl group is oriented towards the the inner side of the ring (Figure 3.5). Additionally, all three localized water molecules are displaced by the bound inhibitor and an epoxyketone specific water molecule is hydrogen-bonded to the $\mathrm{C} 6-\mathrm{OH}$ and to Thr21 $\mathrm{\gamma}$-OH, referred to as $\mathrm{EK}-\mathrm{H}_{2} \mathrm{O}$.

These findings suggest that the ring closure proceeds via a nucleophilic attack by the N-terminal amine of the epoxide $\beta$ carbon and not by the epoxide $\alpha$ carbon, as reported for the 6-ring closure.

A

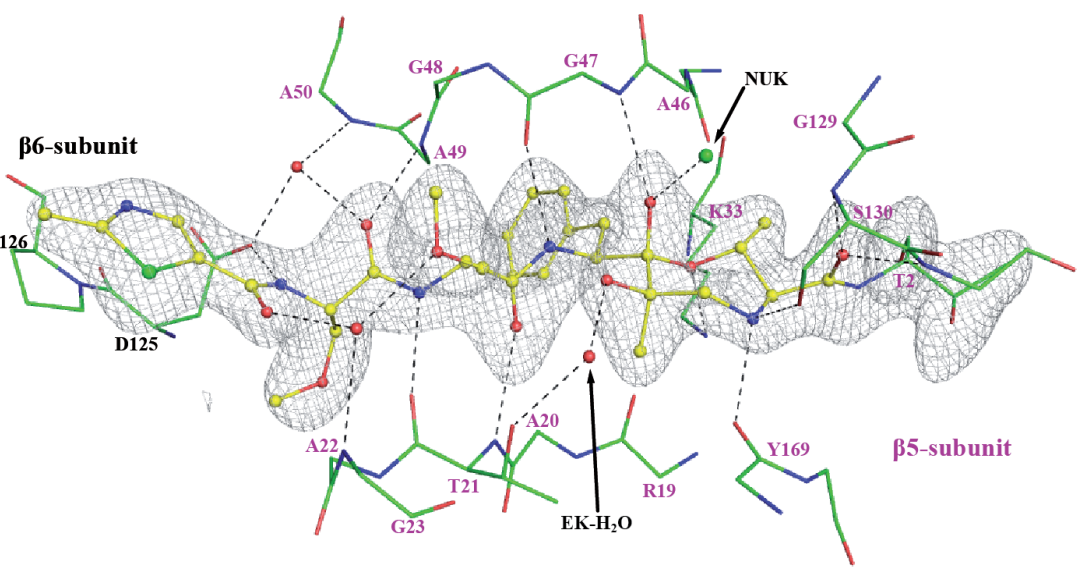

B

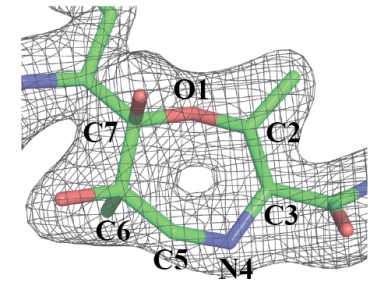

C

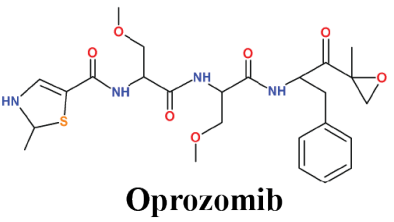

Figure 3.5: High-resolution human 20S proteasome in complex with Oprozomib. (A) The $\beta 5$ active site inhibited by Oprozomib at $1.9 \AA$. The omit map for the inhibitor, the cyclic linkage, and $\beta$ Thr2 is contoured at $4 \sigma$. Depicted side chains are enumerated and the NUK chloride is shown as a green dot. The inhibitor is covalently attached to Thr1 are depicted in ball-and-stick representation. Dashed lines indicate hydrogen bonds at $\leq 3.2 \AA$ distance. (B) Close-up view of the 7-membered ring-closure, along with the corresponding omit map contoured at $6 \sigma$. (C) Chemical structure of Oprozomib. Figure adapted from Schrader et al., $2016^{120}$.

In the following, a multitude of control experiments to validate the 1,4-oxazepane ring formation were carried out: (I) 20S proteasome crystals were grown as described before. After full growth, the crystals were soaked with $\mathrm{Mg}(\mathrm{OAc})_{2}$ prior to soaking with the inhibitor Oprozomib. In the calculated electron density, an exchange of the chloride molecule for an acetate molecule was detected, while the formation of a 1,4-oxazepane linkage was not affected. As seen in Figure 3.6 the electron density in the active site represents the larger 7 -membered ring and an additional acetate molecule is present. This acetate molecule forms hydrogen-bonds to each of the $\mathrm{C6}-\mathrm{OH}$ and the $\mathrm{C} 7-\mathrm{OH}$ of the 1,4-oxazepane ring as well as a hydrogen bond to the amide of the main chain segment Ser130. These findings show that the exchange of chloride for acetate does not affect the formation of a 7-membered, 1,4-oxazepane linkage.

(II) This experiment was performed to ensure that the formation of the 1,4-oxazepane ring linkage is not a consequence of radiochemistry during X-ray data collection. First, 


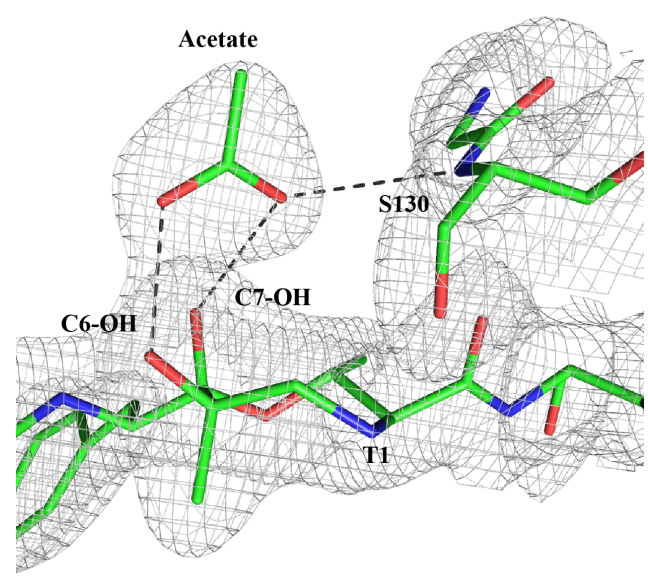

Figure 3.6: Active site of Acetate and Oprozomib soaked $20 \mathrm{~S}$ crystals. Crystals soaked with $\mathrm{Mg}(\mathrm{OAc})_{2}$ before soaking with inhibitor, show the presence of an acetate molecule in the active side together with the covalently linked inhibitor. The close-up view shows the 7-ring structure together with the acetate which is hydrogen-bonded to two oxygen atoms of the 1,4-oxazepane ring. Hydrogen bonds are visualized as dashed lines. Figure adapted from Schrader et al., $2016^{120}$.

a complete dataset at a low X-ray dose (0.7 MGy) was collected (Figure 3.7, A), then the crystal was treated with a high dose (15 mGy) exposure followed by the collection of a second dataset at 0.7 MGy exposure (Figure 3.7, B). The calculated electron density maps revealed no obvious differences and that the 7-ring is not a consequence of radiation damage.

A

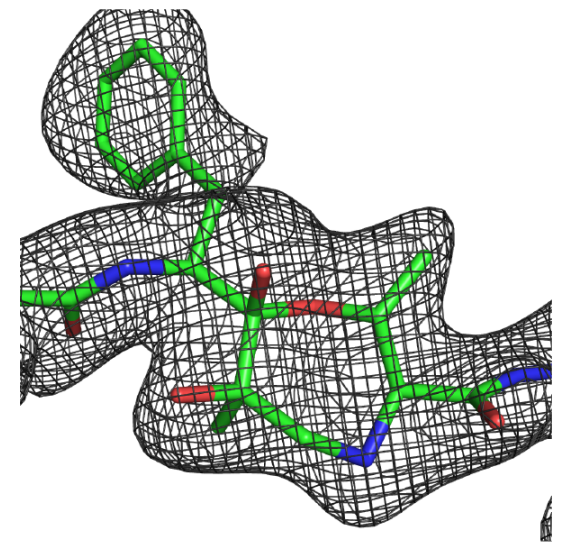

low dose exposure

(0.7 MGy)
B

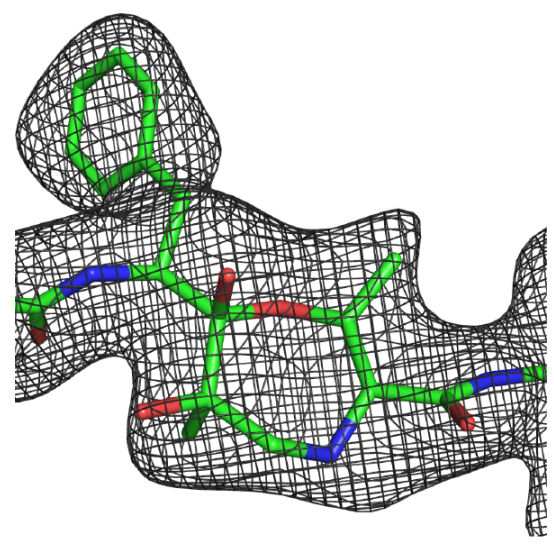

after 15 MGy exposure

Figure 3.7: Low-dose experiment. Shown are the electron density maps of the ring linkage region from a (A) low-dose and (B) a high-dose exposure. Both maps show electron density for the 1,4-oxazepane 7-membered ring and no obvious differences are discernable. Figure adapted from Schrader et al., $2016^{120}$.

(III) Next, crystals were soaked with the natural product epoxyketone, Epoxomicin. Diffraction data to $2.4 \AA$ were collected. The structure was calculated by refinement of the integrated and scaled X-ray data against the present model of the native human 20S. The electron density maps in the human epoxyketone-20S proteasome crystal structure revealed density for the bigger 7-ring linkage formation. Modeling the cyclic molecule visible in the inhibited state reveals that it represents the 1,4-oxazepane ring structure. Figure 3.8 illustrates the $\beta 5$ active site bound by Epoxomicin at $2.4 \AA$ accompanied by 
an omit map for the inhibitor, covalent linkages, and the $\beta 5 \mathrm{Thr} 2$, as well as the chemical structure of Epoxomicin.

A

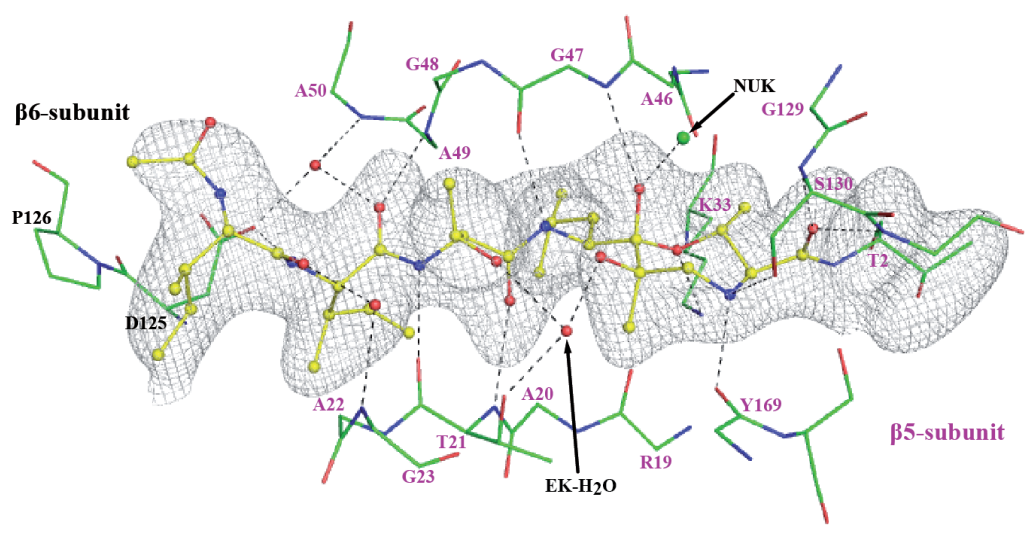

B

C
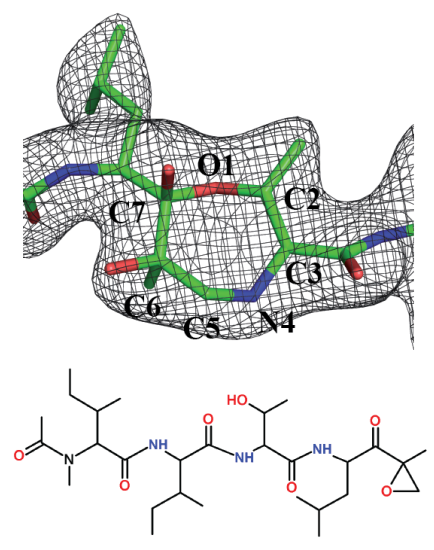

Epoxomycin

Figure 3.8: Epoxomicin-human 20S proteasome co-crystal structure. (A) Shown is the $\beta 5$ active site inhibited by Epoxomicin at $2.4 \AA$. The omit map is contoured at $4 \sigma$. Depicted side chains are enumerated and the NUK chloride is shown as a green dot. Dashed lines indicate hydrogen bonds at $\leq 3.2 \AA$ distance. (B) Close-up view of the 7-membered ring-closure, along with the corresponding omit map contoured at 6 б. (C) Chemical structure of Epoxomicin. Figure adapted from Schrader et al., $2016^{120}$.

(IV) A high-resolution structure of the human 20S proteasome bound by another natural epoxyketone product, Dihydroeponemycin, was determined. In contrast to the previously investigated epoxyketone inhibitors Oprozomib and Epoxomicin, Dihydroeponemycin shows a methanolic group at the $\alpha$ carbon (Figure 3.9, A and B). At a resolution of $2 \AA$ the difference between an $\alpha$ carbon methyl group and a methanolic group should further confirm the given ring-closure. If the binding would result in the formation of a 1,4-morpholine ring, then the calculated structure would reveal two methanolic groups at the carbon 6 position. Instead, the electron density for the Dihydroeponemycin-20S structure represents a 7-ring closure similar to the Epoxomicin and Oprozomib structures. Here, one methanolic group at the $\mathrm{C} 6$ atom oriented to the inner side of the ring is visible (Figure 3.9, C and D).

(V) Since the finding of a 7-ring closure in the inhibited $20 \mathrm{~S}$ proteasome is described for the first time, it was important to assure that the alternative 6-ring formation is not present in the data and the 7-ring is not just one possible ring closure besides the 6 -ring. This question lead to the next experiment, to investigate if the 6-ring formation is an alternative ring-closure form. Therefore, a 6-ring model was refined against the Oprozomib-20S X-ray data. The result, depicted in Figure 3.10, showed a poor quality fit. It forces the structure to adapt the 6-ring formation, which leads to tremendous distortions in the molecular geometry of the model: (i) the N4-carbon bonds are elongated by $0.1-0.2 \AA$, (ii) the C5-alcohol bond is shortened by $0.1 \AA$, (iii) the bond-angle of the C5-methylalcohol differs by -20 degrees from the expected value, (iv) the van-der-Waals 
A

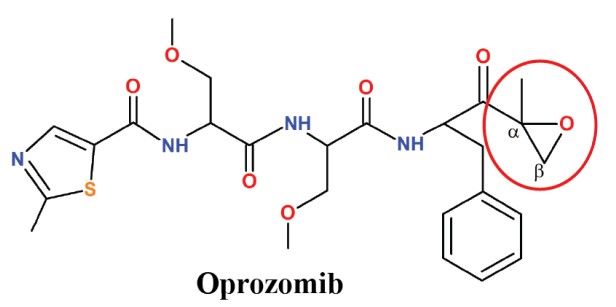

C

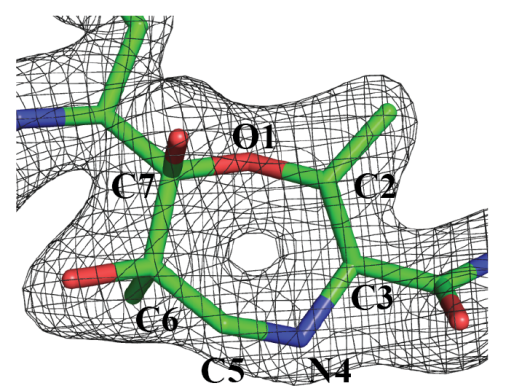

B<smiles>CC(C)CCCCC(=O)NC(CO)C(=O)NC1CC(C)OC(=O)C12CCCCCCCCCC2</smiles>

Dihydroeponemycin

D

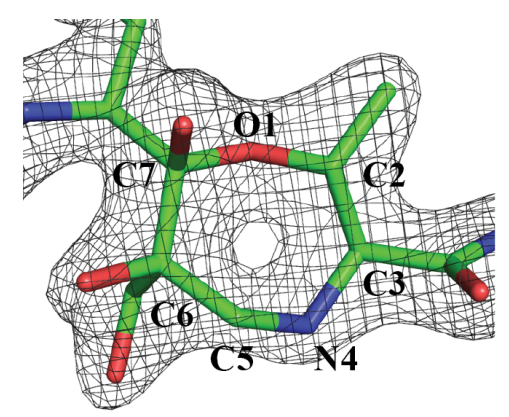

Figure 3.9: Validation of the ring closure in the 20S-Dihydroeponemycin crystal structure. (A) Chemical structure of Oprozomib. (B) Chemical structure of Dihydroeponemycin. In (A) and (B) the red oval highlights the reactive head group: the Oprozomib contains a methyl group and Dihydroeponemycin a methanolic group in the $\alpha$-position to the ketone. (C) Close-up view of the 7 -membered ring-closure upon Oprozomib inhibition, along with the corresponding omit map contoured at $6 \sigma$. (D) Close-up view of the 7-membered ring-closure upon Dihydroeponemycin inhibition, along with the corresponding omit map contoured at $6 \sigma$. The Dihydroeponemycin structure does not reveal electron density consistent with the presence of two methanolic groups.

distance between the C5-methyl alcohol and two main chain oxygen atoms (R19, Y169) is too short with $3 \AA$ and would result in repulsion forces, and moreover, (v) strong negative difference density peaks in difference maps contoured at $5 \sigma$ levels at the C5 methanol oxygen of the 1,4-morpholine ring model, as well as positive density peaks contoured at $4.5 \sigma$ levels close to positions 4 and 5 of the 7-ring model remained after 6 -ring refinement. Adding it all up, the 6-ring formation cannot be considered as an alternative ring-closure upon epoxyketone inhibitor binding.

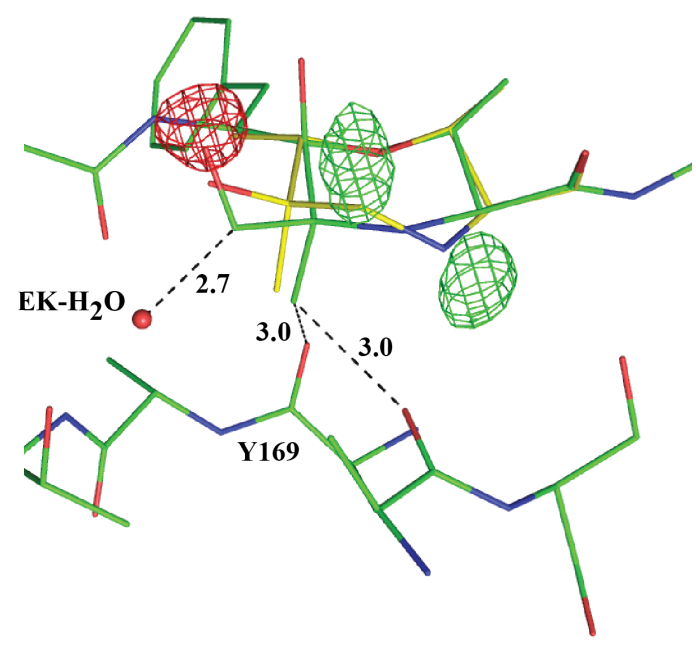

Figure 3.10: Competitive refinement. The competitive alignment of 1,4-morpholine versus 1,4-oxazepane linkage reveals a poor fit and severe distortions can be observed. Shown is an overlay of the active site inhibited by a 7-ring linkage (yellow) and the attempted 6-ring linkage (green). The strong negative difference density is visible at the C5 methanol atom in red, positive densities in green at positions 4 and 5. Figure adapted from Schrader et al., $2016^{120}$. 


\subsubsection{Structural Implications of the Six-ring Linkage}

To investigate structural implications of a real six-ring linkage, a structure of the human 20S proteasome with the inhibitor Z-LLY Ketoaldehyde was determined and analyzed at $2.1 \AA$ resolution. Z-LLY-Ketoaldehyde belongs to the inhibitor class of the $\alpha$-ketoaldehydes, which possess only an electrophile in the $\alpha$ position. It is therefore only capable of forming a 6-membered ring linkage.

The structure revealed that the ring-closure is without a doubt formed by six carbon atoms (Figure 3.11). The electron density at the active site shows a clear density for a hydroxyl group at the C5 position of the linkage, which indicates a formation of a 1,4-morpholine linkage. This is unlike previously published data where the ring closure for $\alpha$-ketoaldehydes was proposed to be a 6 -membered heterocyclic, 5,6 -dihydro- $2 \mathrm{H}-1,4$ oxazine ring.

Considerable differences become visible when comparing the coordination of the presented epoxyketone-inhibited structures forming the 7-ring linkage to the 6-ring forming ketoaldehyde-inhibited structure. First, the NUK chloride molecule is absent and instead a water molecule is present in a new position $1.2 \AA$ away from the NUK chloride side. This water molecule forms hydrogen bonds to the Ser130-amine and $\gamma$-OH as well as to $\mathrm{N} 4$ and $\mathrm{C} 6-\mathrm{OH}$ of the 1,4-morpholine ring. The $\mathrm{C} 5-\mathrm{OH}$ moiety, characteristic for the 1,4-morpholine ring closure, is stabilized by a dense hydrogen-bond network involving the carbonyl atoms of Arg19 and Tyr169, the nitrogen of Lys33, and the active site $\mathrm{H}_{2} \mathrm{O}-3$ (CAT).

These results indicate that the $\mathrm{H}_{2} \mathrm{O}-3$ might play a role in both proteasomal catalysis of peptide cleavage and in the cyclization step of the inhibition reaction. 
A<smiles>CCCCCCCC(=O)C1(NC(=O)[C@H](CC(C)C)NC(=O)[C@H](CC(C)C)NC(=O)OCc2ccccc2)CCCCCCCC1</smiles>

B

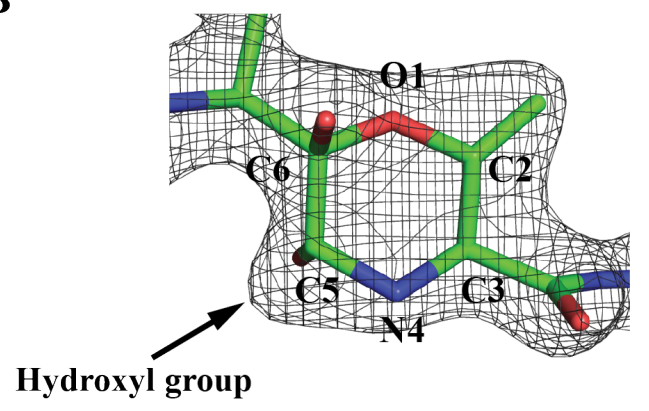

C

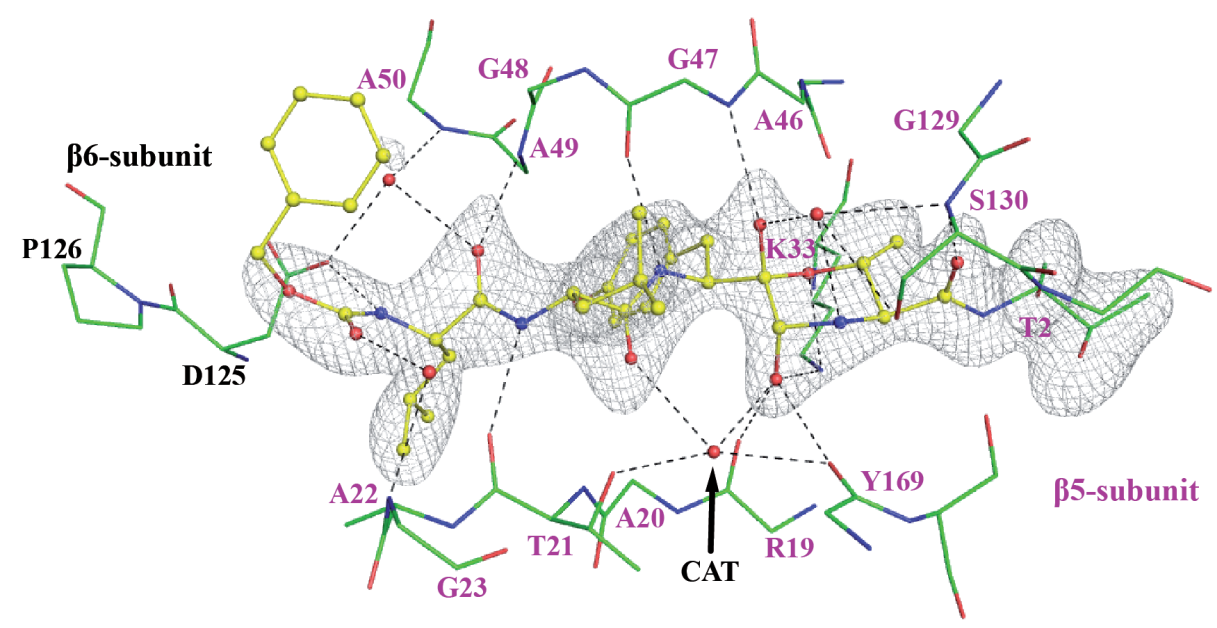

Figure 3.11: Ring-closure in the human 20S proteasome structure in complex with Z-LLYKetoaldehyde. (A) Chemical structure of Z-LLY-Ketoaldehyde. The red circle highlights the reactive head group with the electrophile in the $\alpha$-position. (B) Close-up view of the 6 -membered ring-closure, along with the corresponding omit map contoured at $6 \sigma$. The arrow points to the C5 position where a clear electron density for the hydroxyl group is visible. (C) The inhibited $\beta 5$ active site is illustrated, along with an omit map contoured at $4 \sigma$ for the inhibitor, the cyclic linkage, and $\beta 5$ Thr2. Depicted side chains are numbered. The NUK chloride is shown as a green dot. Dashed lines signify hydrogen bonds ( $\leq 3.2 \AA$ distance). Figure adapted from Schrader et al., $2016^{120}$. 


\subsubsection{Elucidation of the Inhibition Mechanism of Epoxyketone Inhibitors}

To address the hypothesis that the $\mathrm{H}_{2} \mathrm{O}-3$ might play a critical role in the function of the human $20 \mathrm{~S}$ proteasome and in its inhibition, three high-resolution structures of human 20S proteasomes inhibited by boronic acid inhibitors were determined and the active sites were analyzed. Boronic inhibitors were chosen because they mimic the first tetrahedral intermediate state in protein cleavage.

A $2.1 \AA$ structure of the Bortezomib-20S, a $2.0 \AA$ structure of the Ixazomib-20S, and a $2.0 \AA$ structure of the Delanzomib-20S proteasome complex were determined. The details of the site of inhibition are presented in Figure 3.12 for each of these inhibitors. All three calculated structures of the active site do not exhibit a NUK chloride molecule. The NUK chloride is exchanged against a water molecule. In addition, the localized water molecules $\mathrm{H}_{2} \mathrm{O}-1$ and $\mathrm{H}_{2} \mathrm{O}-2$ are displaced by the inhibitor. The $\mathrm{H}_{2} \mathrm{O}-3$ (CAT) molecule remains hydrogen-bonded to the boronic acid moiety, where it might stabilize the tetrahedral intermediate.

Given these observations, it is quite likely that the NUK chloride molecule located on the opposite stereoface of the inhibitor cannot support the cyclization step of $\alpha^{\prime}, \beta^{\prime}-$ Epoxyketone and $\alpha$-Ketoaldehyde inhibitors. In contrast, $\mathrm{H}_{2} \mathrm{O}-3$ (CAT) is well positioned in the active site to support the cyclization of the 6-, or 7-ring, respectively.

Furthermore, on the basis of the crystal structures of the inhibited human 20S proteasome, cluster quantum chemical calculations were performed to better understand the inhibition mechanism. The system of choice is the Dihydroeponemycin inhibitor, with two substantial benefits: the 6- and 7- ring formation can be compared within one system and according to the crystal structure, the spectating hydroxyl moiety of the inhibitor displaces the much more dynamic catalytic $\mathrm{H}_{2} \mathrm{O}-3$.

The previous results indicated that the residues Asp17 and Lys33 cooperate for the activation of the nucleophilic oxygen in Thr1 and the NUK chloride molecule stabilizes the tetrahedral intermediate. The model system depicted for this analysis includes the neighboring residues Lys33, Ser130 and Asp17, the NUK water, and an additional water to complete the hydrogen bond network to Ser130.

Reaction paths were computed and a connected path was obtained: The reaction proceeds via a proton transfer from the Thr1 $\gamma$-OH to the Lys33 $\varepsilon$-amine, followed by a nucleophilic attack of the hydroxide. Two groups are important for the energetics of this pathway: Asp17 and the NUK water. Asp17 favors the formation of the $\varepsilon-\mathrm{NH}_{3}{ }^{+}$group in the lysine and the NUK water forms a strong hydrogen bond with the Ser130 residue. The cluster model is shown in Figure 3.13, A. The electronic energy differences in the computed pathway for the nucleophilic attack on the keto carbon of Dihydroeponemycin 
A

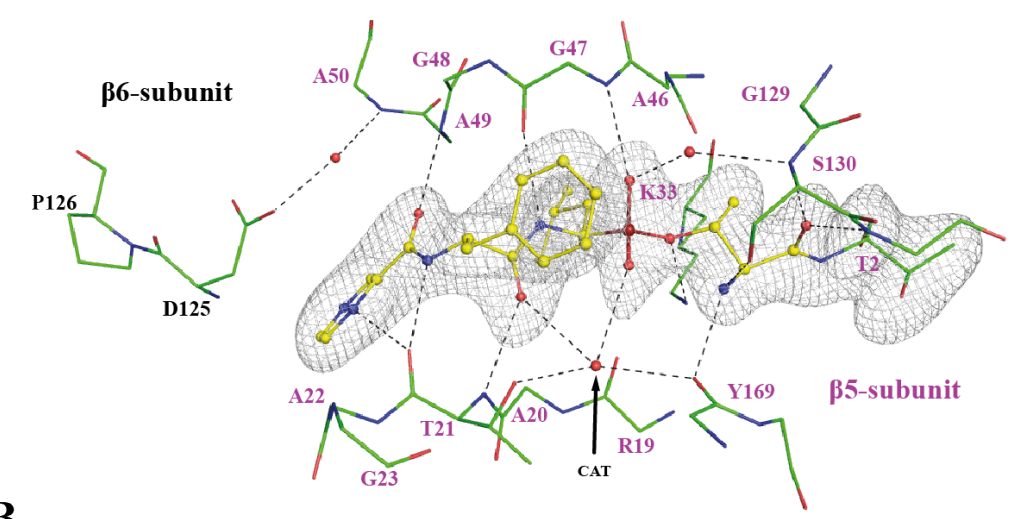<smiles>CC(C)C[C@H](NC(=O)[C@H](Cc1ccccc1)NC(=O)c1cnccn1)C(=O)O</smiles>

Bortezomib

B

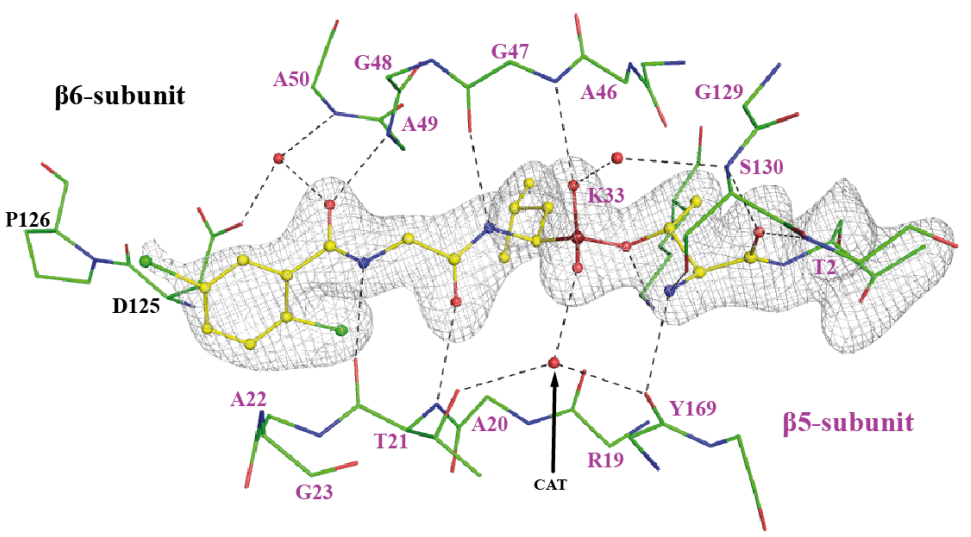<smiles>CC(C)C[C@H](NC(=O)CNC(=O)c1cc(Cl)ccc1Cl)C(=O)O</smiles>

C

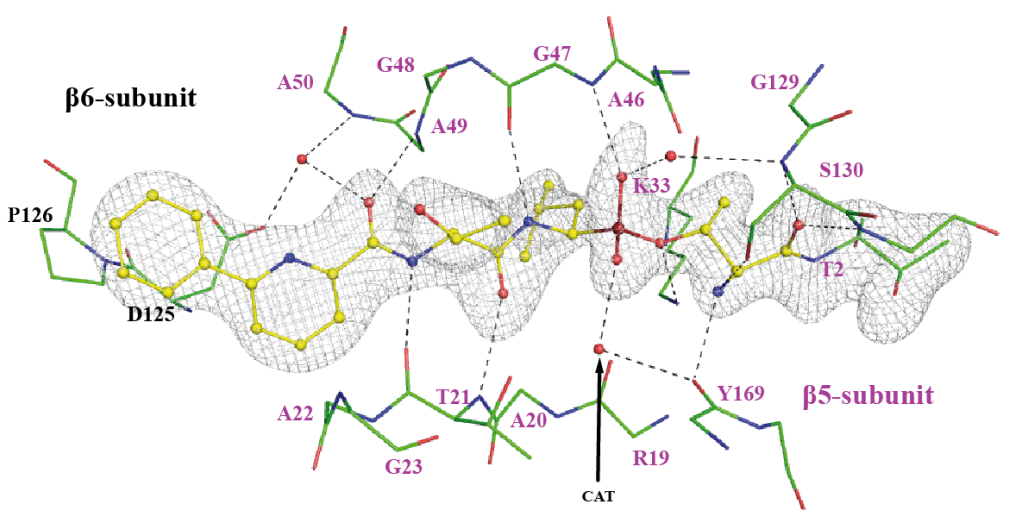<smiles>CC(C)C[C@H](NC(=O)C(NC(=O)c1cccc(-c2ccccc2)n1)C(C)O)C(=O)O</smiles>

Delanzomib

Figure 3.12: Structures of three boronic acid inhibitors bound to the human 20S proteasome. (A) Bortezomib, (B) Ixazomib and (C) Delanzomib. In all three cases, the left panel illustrates the inhibited $\beta 5$ active site of the human $20 \mathrm{~S}$ proteasome. On the right side the omit map contoured at $4 \sigma$ for the inhibitor, covalent linkage and $\beta 5 \mathrm{Thr} 2$ is shown. Depicted side chains are numbered and the NUK chloride is exchanged by a water molecule in all three structures. Dashed lines indicate hydrogen bonds at $\leq 3.2 \AA$ distance. The $\mathrm{H}_{2} \mathrm{O}-3$ (CAT) molecule is shown as a red dot. Figure adapted from Schrader et al., $2016^{120}$.

are shown in Figure 3.14, A.

In a second set of calculations, the energetics for the pathways leading to 6-ring and 7-ring formations were compared. From the Dihydroeponemycin data a model for the active site was chosen (Figure 3.13, B). This model includes the Thr1 amine, the 3-ring of Dihydroeponemycin and one water molecule was added to complete the hydrogen bond network between the Dihydroeponemycin methanolic $\mathrm{OH}$ group, and the Thr1 reactive 
$\mathrm{NH}_{2}$ group. This role is most likely taken over by Ser170 in the native environment. The NUK solvent molecule in addition to the residues Lys33, Ser130 and Asp17 were neglected since the NUK is located on the opposite stereoface of the ring and the residues has little impact. Both pathways were obtained by performing constrained optimizations, keeping the distance between the nitrogen and the target carbon in the inhibitor. The final reaction pathways for the 6- and the 7-ring formation are shown in Figure 3.14. Both pathways originate from the same starting structure that is the tetrahedral intermediate with the amine group facing the epoxide.

Thus, it appears that the reaction's bottleneck is the cyclization step of the inhibition reaction (Figure 3.15). The seven-ring pathway is kinetically favored by approximately $18 \mathrm{~kJ} / \mathrm{mol}$ and the six-ring is thermodynamically more stable (by approximately $30 \mathrm{~kJ} / \mathrm{ml}$ ). Due to the strain of the transition state, the seven-ring formation is more favored.

The calculations gave no evidence that the NUK solvent molecule participates in the cyclization step due to its location and instead, from the computed pathways and the boronic acid inhibited human $20 \mathrm{~S}$ proteasome structure, $\mathrm{H}_{2} \mathrm{O}-3$ might serve as a proton shuttle in the cyclization step as well as in the peptide hydrolysis.

A<smiles>CCC(N)N</smiles><smiles>CC(=O)C1(CO)CO1</smiles>

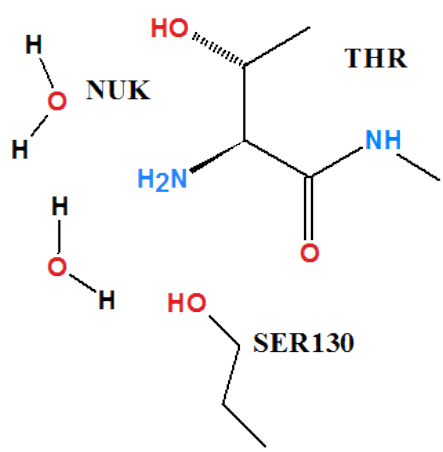

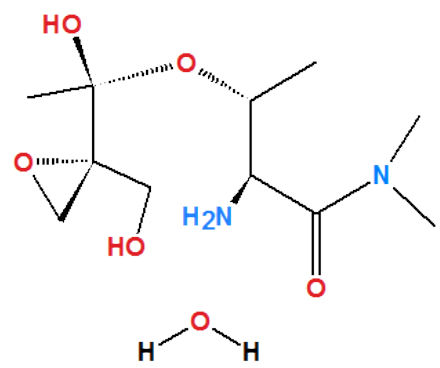

Figure 3.13: Model system used for electronic structure calculations. Model system for (A) tetrahedral intermediate formation and (B) a smaller model system for the ring formation of epoxyketone inhibitors focusing on the Thr1 amine and the 3-ring of the inhibitor, including one water molecule. Figure adapted from Schrader et al., $2016^{120}$. 
A

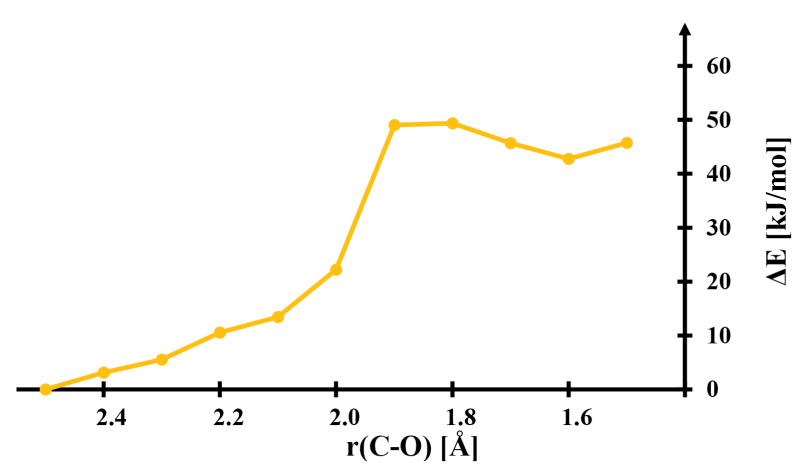

B

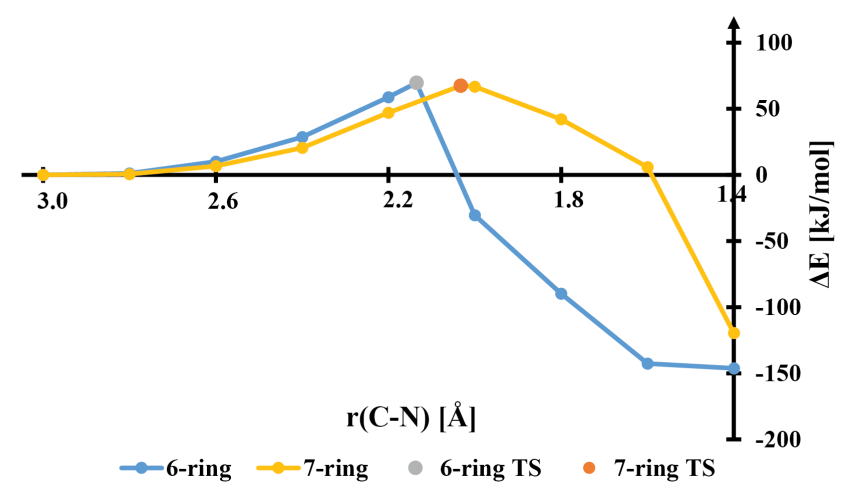

Figure 3.14: Electronic energy differences. (A) Reaction profile of the pathway for the nucleophilic attack at the keto carbon. The $\alpha$ carbons of the capped residues were kept fixed. This profile indicates that Lys33 and Asp17 cooperate to activate the nucleophile and the NUK stabilizes the tetrahedral oxyanion. (B) Full reaction pathways for both six- and seven-ring formations through a similar epoxide ring opening event. The $\mathrm{r}(\mathrm{C}-\mathrm{N})$ distance corresponds to the distance in the forming bond, and therefore refers to two different carbons. Figure adapted from Schrader et al., $2016^{120}$.

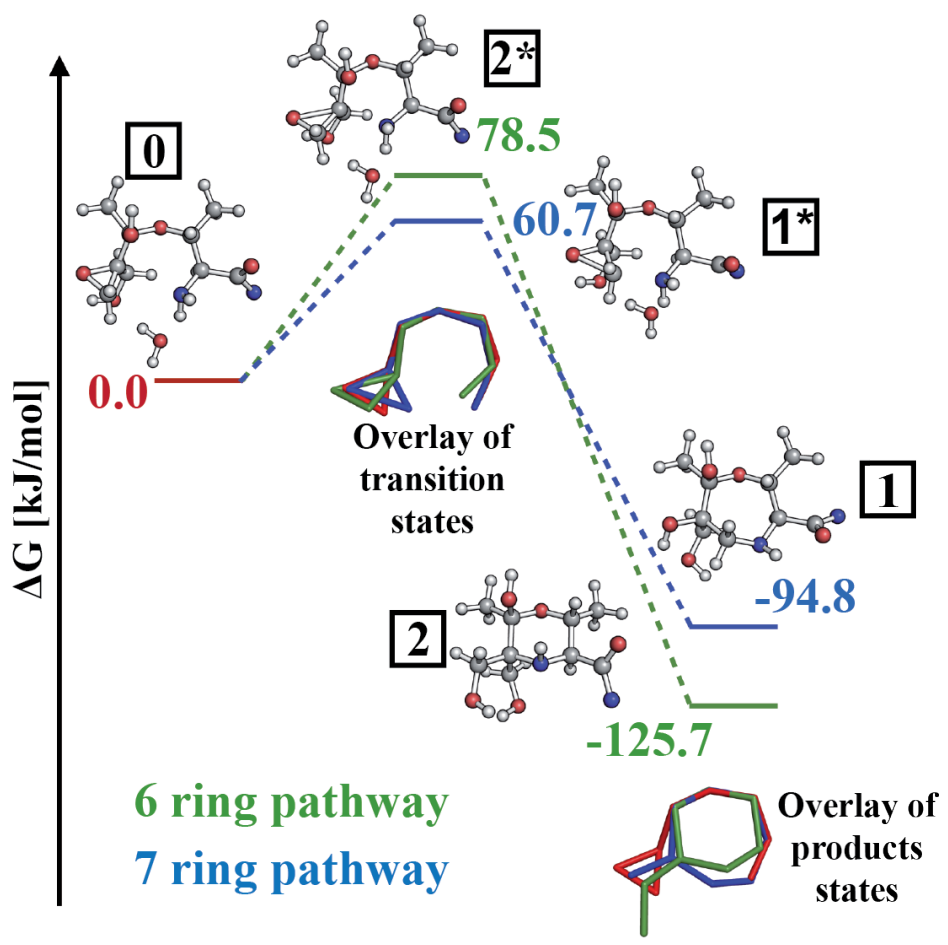

Figure 3.15: Computed reaction pathway of the Dihydroeponemycin model. The starting state structure, the transition state and the final ring-closure state. A schematic overlay of the transition state and the product states compared to the starting structure (red) is included. The seven-ring reaction pathway starts from 0 through transition state $1^{*}$ to 1 . The six-ring reaction pathway starts from 0 though transition state $2^{*}$ to 2 . Figure adapted from Schrader et al., $2016^{120}$. 
Additionally, kinetic measurements were performed to provide more information about the kinetic preferences of the 6- and 7-ring formation, respectively. The analysis of the first-order inactivation rate constants of 6-ring forming Z-LLY-Ketoaldehyde compared to the 7-ring forming Dihydroeponemycin and Oprozomib indicates that the six-ring formation rate is smaller by a factor of approximately 20 (Figure 3.16). Further, the residual rate of $3 \%$ of the maximum activity detected for both Dihydroeponemycin and Oprozomib confirms that the seven-ring product is thermodynamically less stable. This results in a partially reversible inhibition for epoxyketone inhibitors, which corroborates the observations made by cluster quantum calculations.

Figure 3.16: Kinetic analysis of inhibitor binding. Shown are the kinetic analysis of the first order inactivation rate constants for 6-ring forming Z-LLYKetoaldehyde and 7-ring forming epoxyketone inhibitors Dihydroeponemycin and Oprozomib. The analysis of the Ketoaldehyde inhibitor showed no residual activity, indicating an irreversible inhibition mechanism. For the epoxyketones, the residual activity of approximately $3 \%$ indicates a partial reversible inhibition. Figure adapted from Schrader et al., $2016^{120}$.

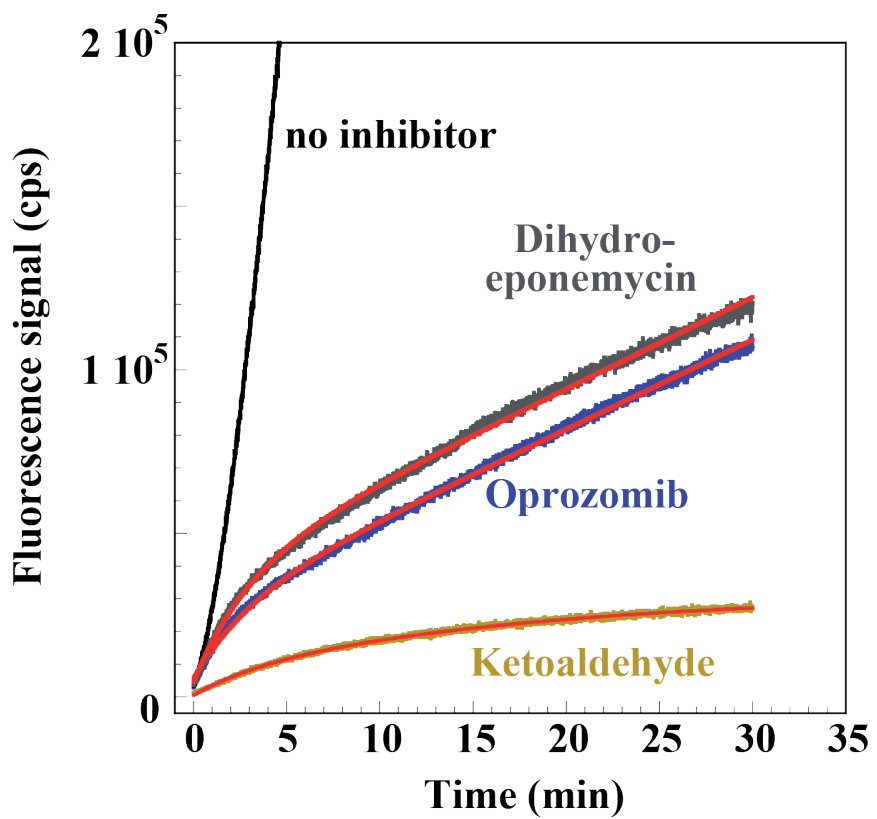




\subsection{Structural Impact of 20S Inhibitors on the Human Proteasome Holoenzyme}

The first part of this thesis focuses on the human 20S proteasome. A large number of crystal structures of human $20 \mathrm{~S}$ proteasomes in complex with inhibitors were studied, largely because $20 \mathrm{~S}$ inhibition is an established approach for cancer treatment and much is unknown about their inhibition mechanisms ${ }^{120}$. Proteasome inhibitors have a 20 year history in cancer therapy ${ }^{144}$ but the structural impact of $20 \mathrm{~S}$ inhibitors on the fully assembled 26S proteasome remains almost elusive.

The findings of the inhibition mechanisms cleared the picture about the competitive inhibition of the human $20 \mathrm{~S}$ proteasome. The information gained from the crystal structures will help to design new inhibitors and improve their effectiveness.

The mystery about proteasome inhibition remains for the regulatory particle of the proteasomal holoenzyme. Does the inhibition affect the 19S particle? Is the inhibition represented by a structural rearrangement or restricted movements within the proteasome? One publication from 2007 provides a first indication that proteasome inhibitors are potent in stabilizing $26 \mathrm{~S}$ proteasomes against dissociation of the core particle and the regulatory particle ${ }^{145}$.

In the following sections, a structure of the human $26 \mathrm{~S}$ proteasome in complex with the 20S proteasome inhibitor Oprozomib is presented and the structural effects of inhibition are analyzed.

\subsubsection{Purification and Analysis of the Human 26S Proteasome in Complex with Oprozomib}

Originating from the previously established biochemical method to purify functional human 20S proteasomes, a purification strategy for 26/30S proteasomes was established and is depicted in Figure 3.17. Other than for 20S proteasomes, the 26S proteasome $23-30 \%$ PEG400 cut was loaded onto sucrose cushions to gain a concentrated 26S proteasome fraction. Following this step, the purification continues in the same way as for the $20 \mathrm{~S}$ proteasomes, reproducibly yielding $15 \mathrm{mg}$ human $26 \mathrm{~S}$ proteasomes, starting from $300 \mathrm{ml}$ cytosolic HeLa extract at a concentration of $10 \mathrm{mg} / \mathrm{ml}$.

By employing isotonic (low salt) conditions throughout the purification, stability of the complex is maximized and the sample is stable at $4{ }^{\circ} \mathrm{C}$ for several weeks as validated by SDS-PAGE analysis and EM preparations. Most human proteasomes purified by this method appear to be in the double-capped 30S form. For structural analysis, only single- 


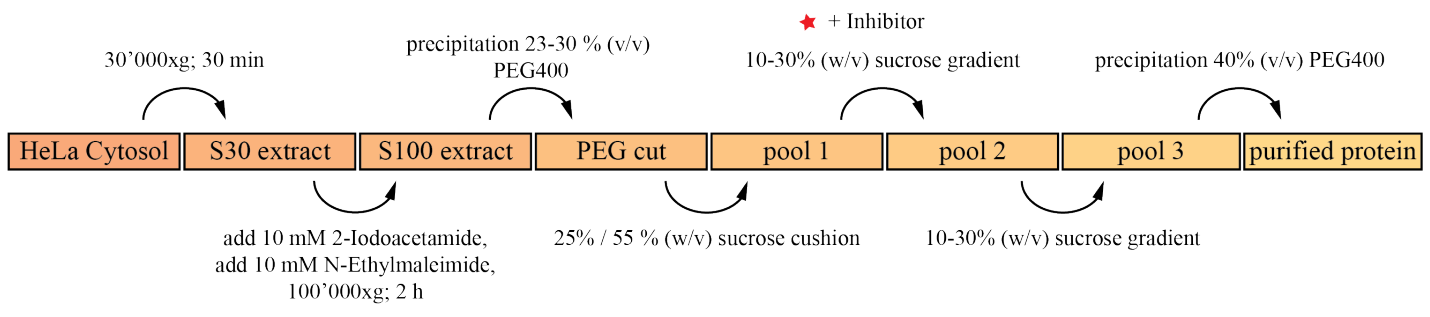

Figure 3.17: Schematic representation of the human 30S proteasome purification. (A) Schematic representation of the entire high-throughput pipeline to purify human $26 \mathrm{~S}$ proteasomes from HeLa cytoplasm. The purification is completed within 72 hours.

capped 26S proteasomes were used to avoid symmetry problems during image processing and to provide a better angular distribution. Figure 3.18, A shows a representative micrograph.

To study the effect of inhibitors on the $26 \mathrm{~S}$ proteasome, the epoxyketone inhibitor Oprozomib was chosen and introduced to the proteasome sample at an early stage during purification. The proteasome pool after sucrose cushion centrifugation was treated with $0.5 \mathrm{mM}$ Oprozomib and incubated for 30 minutes at $25{ }^{\circ} \mathrm{C}$ before continuing with the sucrose gradient centrifugation.

A

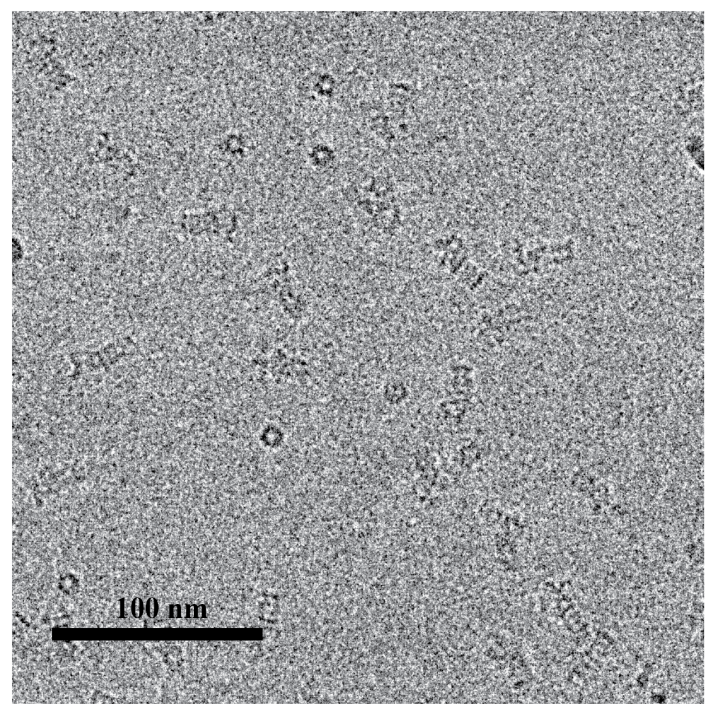

B

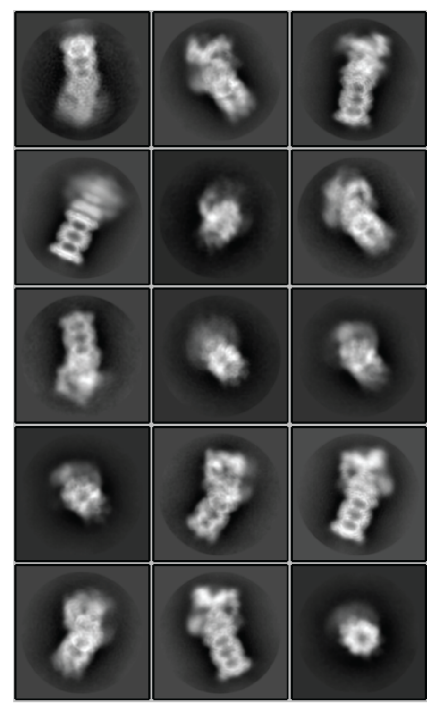

Figure 3.18: Representative micrograph and 2D class averages. (A) Representative cryo-EM micrograph depicting various orientations of Oprozomib-inhibited human 26S proteasomes. By following the purification and cryo-EM preparation procedure described above, high quality micrographs can be obtained. (B) Typical 2D class averages of human Oprozomib-26S proteasome particles. Figure adapted from Haselbach et al., $2017^{146}$.

As part of the ubiquitin-proteasome system, the $26 \mathrm{~S}$ proteasome detects and binds polyubiquitinated substrates. Earlier publications point to the fact that inhibition of the $20 \mathrm{~S}$ proteasome leads to a stabilization and jamming of polyubiquitinated substrates in the 
19S particle ${ }^{147}$. Free ubiquitin or substrate-bound ubiquitin that is attached to the purified proteasome sample might alter the dynamic behavior and therefore the EM data analysis of the molecular machine. Before analyzing the sample on a structural level, we evaluated if the inhibited and non-inhibited samples behave differently in relation to ubiquitin binding. By western blot analysis, we could evaluate if substrate release, hence ubiquitin release is altered in the inhibited sample. For this, the inhibited and the non-inhibited sample were analyzed by SDS-PAGE in equal amounts and subsequently blotted in a western blot chamber against ubiquitin. Figure 3.19 shows the SDS-PAGE and western blot results. Both samples, the inhibited (Oprozomib) and the non-inhibited sample (Native), show antibody signals for ubiquitin (Figure 3.19, lane 3 and 4) but both samples have approximately the same amount of signal and no evidence for accumulation of ubiquitin molecules in the inhibited sample can be confirmed. Thus, it is unlikely that polyubiquitinated substrates or free ubiquitin have an influence on the structural behavior of the inhibited human $26 \mathrm{~S}$ proteasomes.

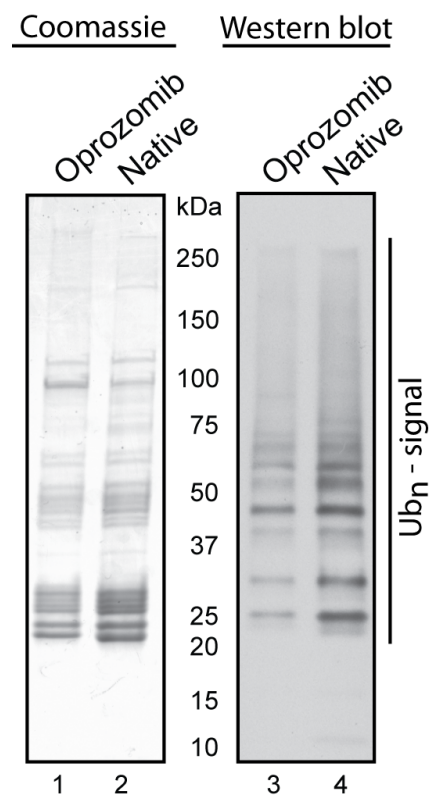

Figure 3.19: Western blot analysis. Purified Oprozomib-inhibited and non-inhibited 26S proteasomes were analyzed by SDS-PAGE and Ubiquitin was detected via western blot analysis. Lane 1 and 2 show Coomassie stained SDS-PAGE gel signals for 26S proteasome proteins. Lane 3 and 4 represent the corresponding Ubiquitin signals detected by western blot analysis. Figure adapted from Haselbach et al., $2017^{146}$. 


\subsubsection{EM Structure of the Human 26S Proteasome in Complex with Oprozomib}

EM sample preparation, data collection and image analysis was performed on Oprozomibinhibited human $26 \mathrm{~S}$ proteasomes. First, the freshly purified sample was inspected for quality and particle distribution by negative stain electron microscopy. Subsequently the sample was prepared for electron cryomicroscopy and 18991 EM micrographs were recorded. Figure 3.18, B shows reference-free 2D class averages after several image sorting steps.

To obtain a high-resolution structure extensive hierarchical computational sorting was performed and only particles which represent the major conformational population were included. The results after various computational sorting steps are shown in Figure 3.23. Finally, a cryo-EM structure of the Oprozomib-inhibited human 26S proteasome at a resolution of $3.8 \AA$ was calculated (Figure 3.20, A) and the corresponding model was built (data statistics in Table 3.3). A look into the details of the inhibited $26 \mathrm{~S}$ proteasome model reveals well-defined densities for the holoenzyme with amino acid side chains in the most stable parts of the molecule and only small variations in local resolution (Table 3.3 and Figure 3.22, A).

The best resolved regions are found in both $\beta$ subunit rings and in the regulatory particle bound $\alpha$ subunit ring of the $20 \mathrm{~S}$ core particle at a resolution of about $3.5-4.5 \AA$. The regulatory particle regions are more flexible, and the resolution decreases to the lowest resolution of $6 \AA$ in the most outer parts of the lid with increasing distance from the rigid parts of the particle. Additionally, a clear density for the inhibitor is visible in the $\beta 5$ active site of the Oprozomib-20S complex and the ligand was modeled into the map (Figure 3.20, A).

The structure was investigated with regards to the B-factor values for all individual residues. The B-factors were analyzed in segments of five amino acids. Three quality levels were defined to validate the model building. In the final 3D model, side chains were modeled in areas with a B-factor smaller than $110 \AA^{2}$. Areas with a B-factor between $110 \AA^{2}$ and $150 \AA^{2}$ were truncated to polyalanine. Residues with B-factors greater than $150 \AA^{2}$ were not included in the final map. Figure 3.22 shows the final human 26S proteasome model colored according to the B-factor value. The B-factors range from $25 \AA^{2}$ (low B-factor) to $175 \AA^{2}$ (high B-factor). The local resolution differences of the EM density map and B-factor distribution of the atomic model are well-correlated. 
A

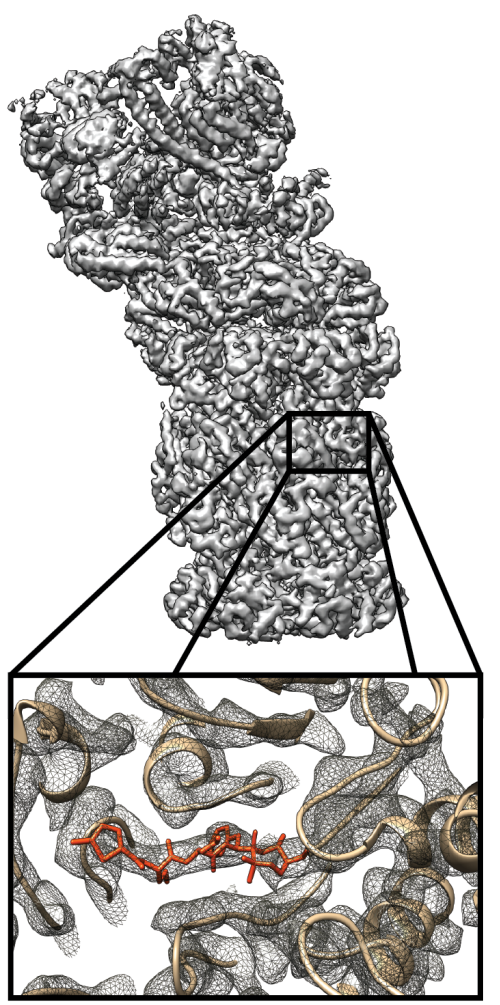

B

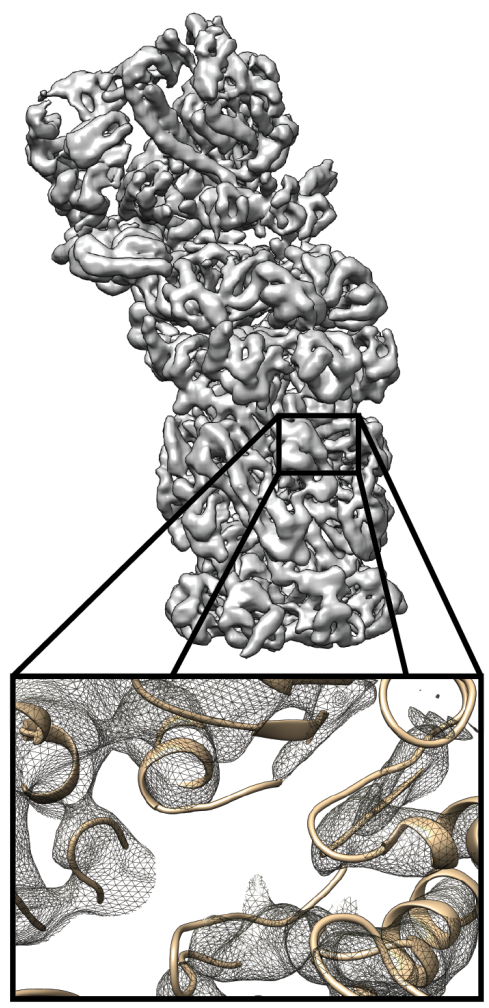

Figure 3.20: Surface view and $\beta 5$ active side of Oprozomib-inhibited and non-inhibited $26 \mathrm{~S}$ proteasome. (A) Oprozomib-inhibited, (B) Non-inhibited. In both cases, the upper panel shows the surface view of the human $26 \mathrm{~S}$ proteasome complex and the lower panel shows a zoom-in to show the $\beta 5$ active site. The inhibited structure reveals an extra density for the inhibitor in the $\beta 5$ active site. Figure adapted from Haselbach et al., $2017^{146}$.

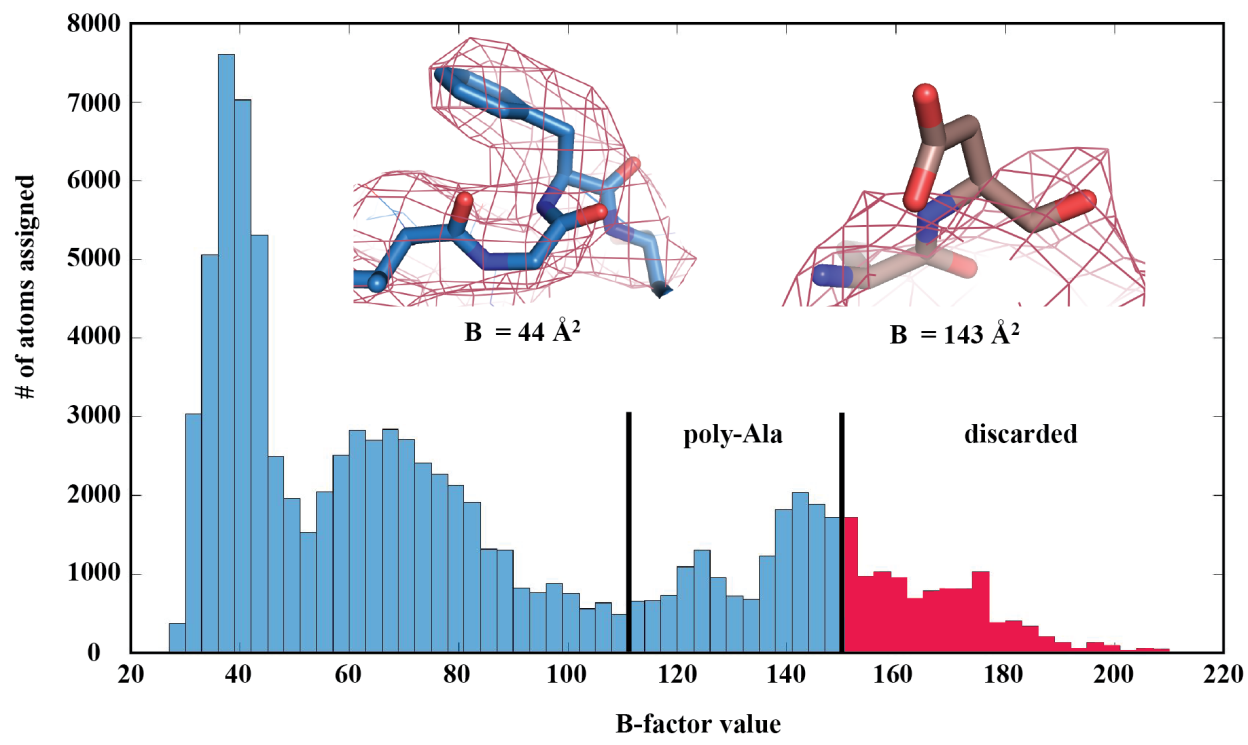

Figure 3.21: B-factor distribution The final human $26 \mathrm{~S}$ proteasome model analyzed for B-factor distribution. In segments of five amino acids the whole structure was analyzed and three quality levels were chosen to define the level of structural details which are interpreted in the final map. Side chains were included in areas with a B-factor value of $110 \AA^{2}$ or less. Only polyalanine chains are shown in areas of B-factors between $110 \AA^{2}$ and $150 \AA^{2}$. No residues were modeled in areas with B-factors higher than $150 \AA^{2}$. Figure adapted from Haselbach et al., $2017^{146}$. 
Table 3.3: Human Oprozomib-26S proteasome model statistics.

\section{Data collection}

Particles

Pixel size $(\AA)$

Defocus range (um)

Electron dose $\left(\mathrm{e}^{-} / \AA^{2}\right)$

$0.4-8$ (mean 1.9)

Refinement

Space group

40.2

a, b, c $(\AA)$

P1

$\alpha, \beta, \gamma\left(^{\circ}\right)$

Resolution $(\AA)$

426.27

Wilson B $\left(\AA^{2}\right)$

$90.0,90.0,90.0$

3.8

66.3

Map sharpening B-factor $\left(\AA^{2}\right)$

Resolution at FSC $=0.143(\AA)$

3.83

$\mathrm{r}_{\text {work }}$

0.4526

Rachmachandran statistics

Outliers

Favored

R.m.s deviations

Bond length $(\AA)$

$2.46 \%$

$83.02 \%$

Bond angles $\left(^{\circ}\right)$

0.015

Validation

EMRinger score

1.669

1.94

A

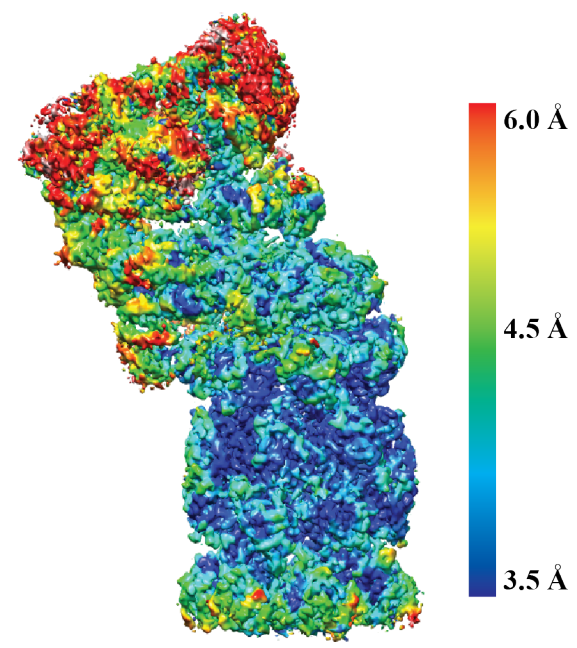

B

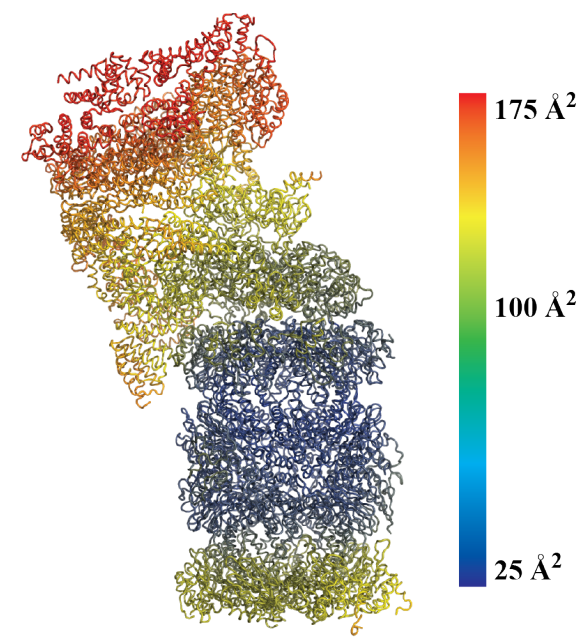

Figure 3.22: Local resolution map and atomic model colored according to the B-factors. (A) Local resolution of the density map of the Oprozomib-inhibited 26S proteasome complex. Resolution ranges from $3.8 \AA$ (blue) to $6 \AA$ (red). (B) Model colored according to the B-factors of all individual residues. B-factors from $25-175 \AA$ are colored. Only single residues show higher flexibility. Figure adapted from Haselbach et al., $2017^{146}$. 


\begin{tabular}{|lr|}
\hline Cryo Statistics & \\
Micrographs recorded: & 18.991 \\
& \\
Picked particles: & 2.521 .909 \\
after CTF \& cleaning: & 924.068 \\
\hline
\end{tabular}

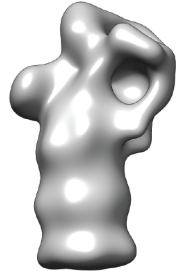

simpe PRIME starting model

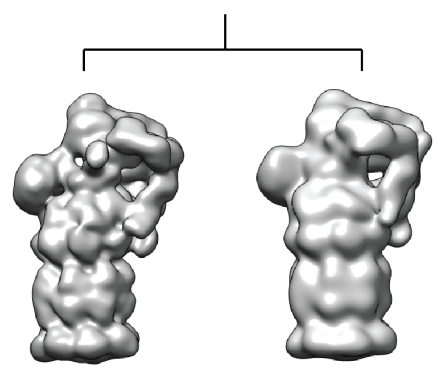

$14.9 \%$ rotated
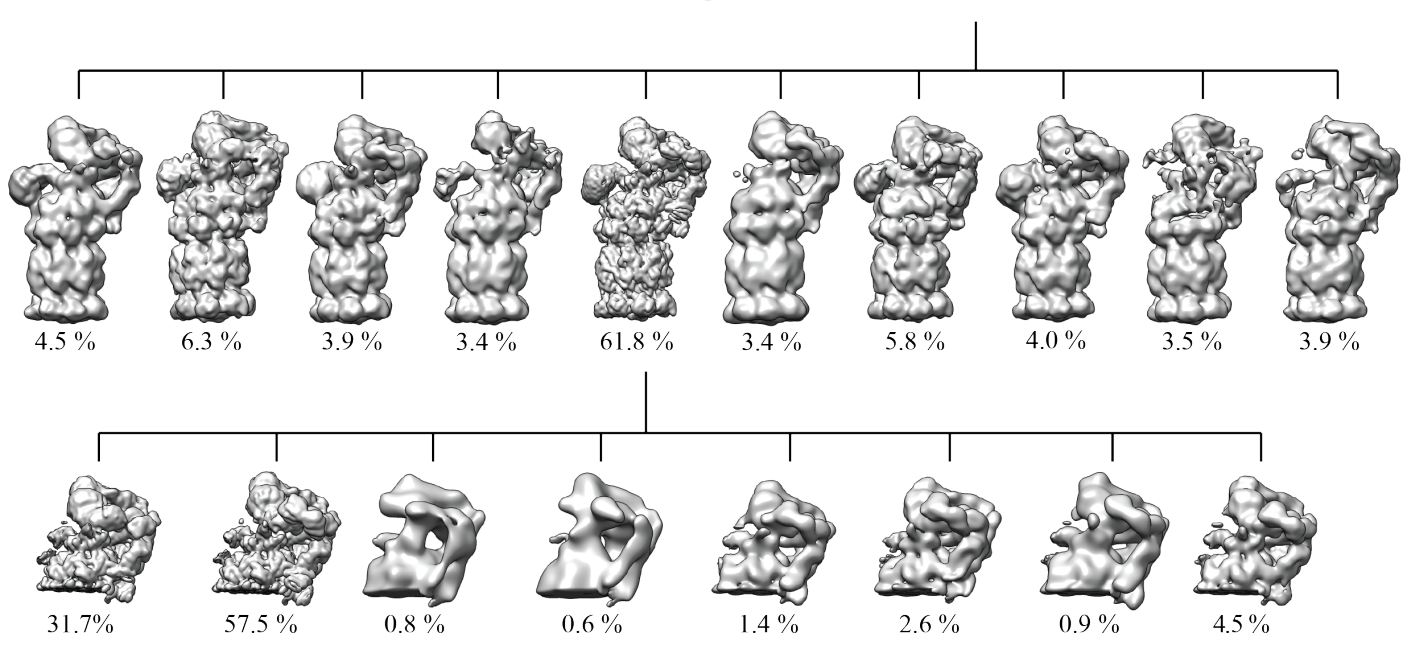

$31.7 \%$
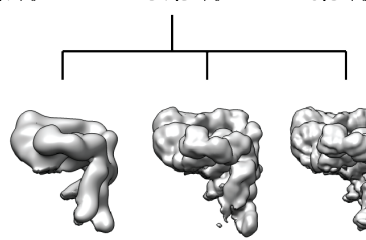

$4.5 \%$

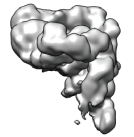

$11.5 \%$
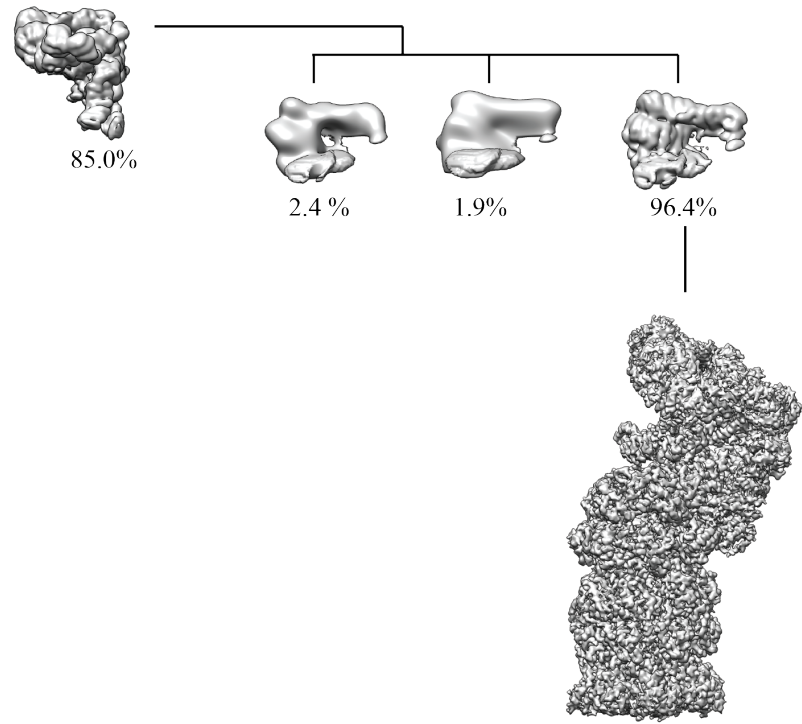

Figure 3.23: Particle sorting scheme. The various computational image sorting steps of the structure determination of the $3.8 \AA$ resolution Oprozomib-inhibited human $26 \mathrm{~S}$ proteasome. In a first step, an initial 3D model was calculated with the software simple PRIME ${ }^{116}$. Next, 3D classification was performed and the particles were sorted according to the two main rotational conformations of the regulatory particle: non-rotated and rotated. Particles contributing to the non-rotated state were further classified in three consecutive rounds of classifications. In each round the highest populated class was chosen and a focused classification was performed. First, with a mask for the complete regulatory particle, second, for the lid and lastly, a mask for the Rpn2 protein. The particles remaining after all steps of classification were refined in RELION ${ }^{115}$ to the final model at $3.8 \AA$. Figure adapted from Haselbach et al., $2017^{146}$. 


\subsubsection{Restricted Dynamics in the Inhibited 26S Proteasome}

Compared to structures from the literature, the overall conformation of the inhibited $26 \mathrm{~S}$ proteasome appears to be similar as no significant differences are visible. To address differences between the inhibited and non-inhibited 26S proteasomes in more detail, a non-inhibited sample was prepared and the structure was calculated at a resolution of 4.8 A. For calculation, the identical image processing and classification protocol was utilized (Figure 3.23).

As seen in Figure 3.20, B, the active site of the non-inhibited human 26S proteasome map shows no extra density and no ligand is bound.

Two major conformational states of the human $26 \mathrm{~S}$ proteasome were identified during data processing for either inhibited or non-inhibited 26S proteasomes (Figure 3.23). These states agree well with previously published conformational states observed for the $26 \mathrm{~S}$ proteasome from yeast ${ }^{50}$. In the following, the conformational states are labeled nonrotated and rotated. This categorization is defined by a rotation of the complete lid by $25^{\circ}$ with respect to the $20 \mathrm{~S}$ core particle combined with a motion of the ATPase Rpt $4 / 5$ coiled coil towards the ubiquitin receptor Rpn10. A schematic representation of the regulatory movement with respect to the $20 \mathrm{~S}$ core particle is shown in Figure 3.24, A.

An analysis of all particles of the native human 26S proteasome shows that $60 \%$ of the particles classify into particle classes representing the non-rotated $26 \mathrm{~S}$ proteasome and $40 \%$ into classes representing the rotated conformation. Applying the same analysis to the particles of the Oprozomib-inhibited 26S proteasome reveals a surprising result. Here, $90 \%$ of all particles represent the non-rotated state and only $10 \%$ are found in the rotated state (Figure 3.24, B).

These findings lead to the question whether binding of the inhibitor stabilizes the nonrotated state. To address this, a control experiment was performed. A freshly purified human $26 \mathrm{~S}$ proteasome sample was treated with the epoxyketone inhibitor Epoxomicin, executing the same inhibition mechanism as Oprozomib. Negative stain electron microscopic analysis and subsequent particle sorting reveals that most particles are in the non-rotated state, as seen for the Oprozomib-inhibited 26S proteasome (Figure 3.24).

These results indicate that inhibition by epoxyketone inhibitors leads to a stabilization of the non-rotated state. $90 \%$ of the inhibited proteasome particles are in the substratebinding state and only $10 \%$ in the rotated, substrate-processing state.

The human proteasome inhibited with epoxyketone inhibitors appears to be restricted in its dynamics which leads to this conformational limitation has a direct connection with the inhibitory effect of epoxyketone inhibitors. 
A
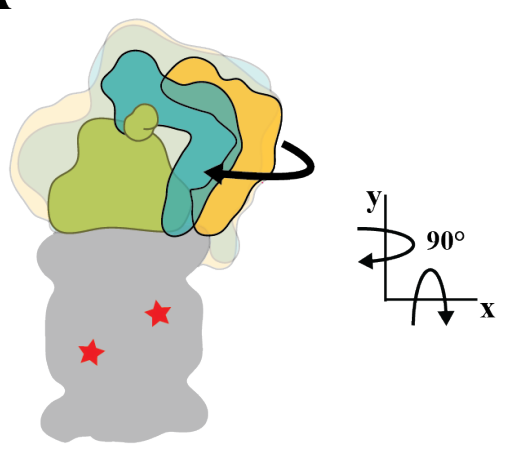

B

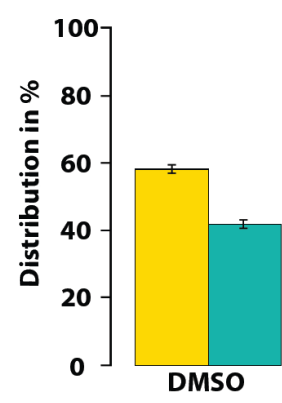

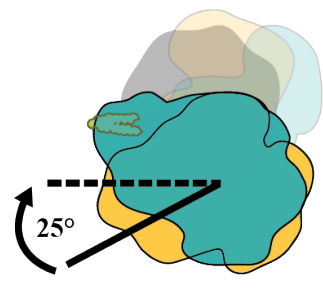

non-rotated

rotated

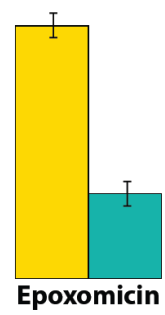

Figure 3.24: Schematic representation of 19S motion and particle distribution. (A) Simplified illustration of the rotation of the lid by $25^{\circ}$ in respect to the $20 \mathrm{~S}$ core particle and movement of Rpt $4 / 5$ towards the ubiquitin receptor Rpn10. The red asterisk marks the $\beta 5$ active side. (B) Bar chart of the particle distribution between non-rotated and rotated state in three datasets: DMSO (control), Oprozomib (epoxyketone), Epoxomicin (epoxyketone). Figure adapted from Haselbach et al., $2017^{146}$. 


\subsubsection{The Energy Landscape of the Lid Movements}

Only a relatively small subset of particles was used to obtain the non-inhibited and Oprozomib-inhibited proteasome maps at 4.8 and $3.8 \AA$ (4\% and $12 \%$ respectively). The final non-inhibited and the Oprozomib-inhibited human 26S proteasome maps adopt a nearly identical non-rotated conformation. This leads to the hypothesis that the differences between the inhibited and non-inhibited $26 \mathrm{~S}$ proteasome are manifested in those particles not used for the high-resolution reconstruction. A novel method to extract valuable information about the dynamic properties of the proteasome and the conformational stabilization elicited by drug binding is presented next.

To make use of the concealed information of all particles which were not part of the final 3D model, a method to study the conformational variability in a quantitative manner was created. In a first step, extensive 3D classification was performed. The classification resulted in 346 classes, 224 of the non-inhibited 26S Proteasome and 122 of the inhibited. Each of the 3D volumes was then refined independently. The final 3D volumes were aligned on the core particle using UCSF Chimera, low-pass filtered, and normalized prior to analysis. To study the conformational variability in the regulatory particle, alignment on the core particle is necessary.

To analyze the motions of the regulatory particles of final 3D classes, Principal Component Analysis (PCA) was used: PCA is a statistical analysis by which a set of observations of possibly correlated variables is converted into a set of values of linearly uncorrelated variables, the principal components or eigenvectors. PCA can be used to reduce the complexity of a data set, resulting in a reduced dataset with only basic information.

Here, the resulting eigenvectors describe the largest covariance which represents the primary movement within the regulatory particle. Analysis of the particle motion was concentrated on the first two modes of motion: The first mode corresponds to the lid rotation with respect to the $20 \mathrm{~S}$ particle and the second mode describes a more complex rearrangement in the lid. In Figure 3.25 (B), the modes are depicted in a simplified way.

With the known particle numbers corresponding to each mode, the transformation of a conformational landscape into an energy landscape, which describes the complexity of the regulatory particle movement in a quantifiable manner, is possible. Additionally, this method allows the direct visualization of changes in the 26S proteasome dynamics upon inhibitor binding.

The results of this analysis are the energy landscapes shown in Figure 3.25. The energy landscapes have to be interpreted according to the coloring. Blue areas represent many particles and a low energy state at the same time. In contrast, red areas have less particles but higher energy states.

Comparing the energy landscapes of the non-inhibited and the Oprozomib-inhibited human 26S proteasome, significant differences become visible: For the Oprozomib-inhibited 
human 26S proteasome, the energy landscape shows wide areas with only few particles but very high energy (red) and a defined blue area with high numbers of particles and low energy. In contrast, the energy landscape of the non-inhibited particles appears to be more flat, represented with only little areas in dark red or blue. The particles have the capacity to adopt conformations on a large scale and no accumulation of particles in one defined area is visible.

These findings indicate a significant limitation in the proteasome's motion upon inhibition. Inhibition of the 20S particle seems to influence the lid motion and the energy landscape reflects a significantly decreased lid movement by creating an energy barrier. It is less likely that the molecules reach the rotated state (red area) and more particle images populate the thermodynamically favored conformation in the blue area, having this energy barrier. In total, only $14 \%$ of the analyzed proteasome particles of the inhibited sample contribute to the red area, hence they represent the rotated state. In addition, we found that the lid rotations of the inhibited sample and the non-inhibited sample differ in the maximum degree of rotation: In the inhibited sample, particles with a lid rotation of maximal $20^{\circ}$ are found, whereas particles of the non-inhibited sample represent a maximum rotation of $25^{\circ}$.

The structural consequences of this smaller amplitude of lid rotation in inhibited $26 \mathrm{~S}$ proteasomes concentrate on the regulatory particle domain orientation. In the non-inhibited $26 \mathrm{~S}$ proteasome, the coiled coil of the AAA + ATPase domains Rpt4/5 reach the interface between the domains Rpn10 and Rpn9. Interestingly, upon a lid rotation of only $20^{\circ}$ the interface is not reached and only Rpn10 is in contact to the coiled coil. This limited rotation might has far-reaching consequences on the proteasome's function. 
A
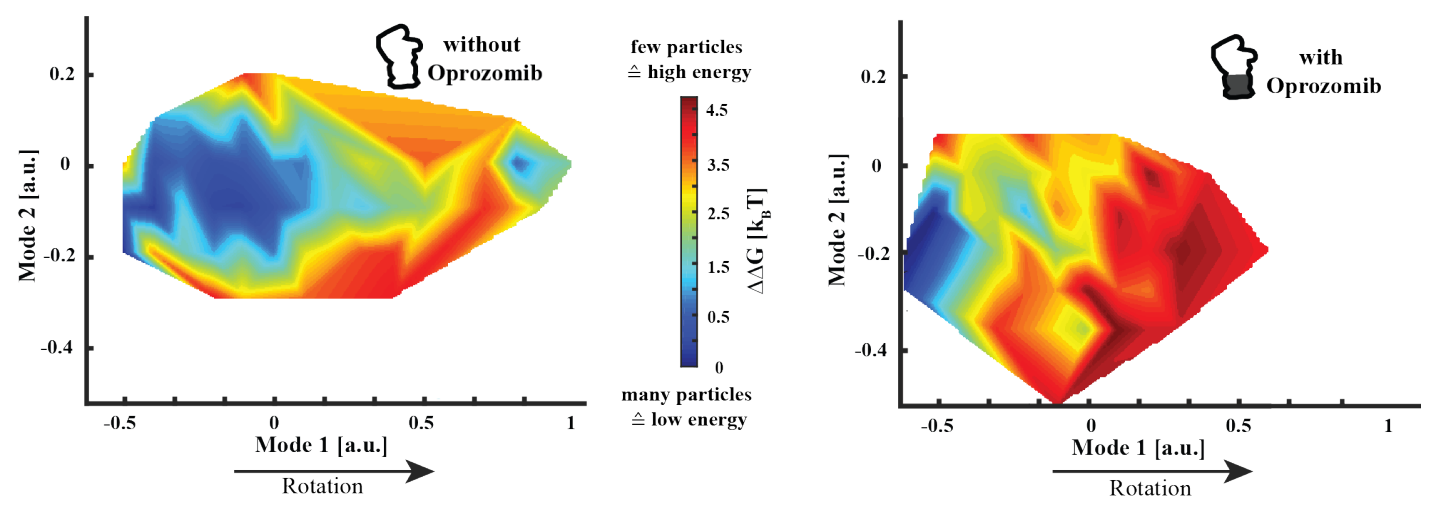

\section{B}
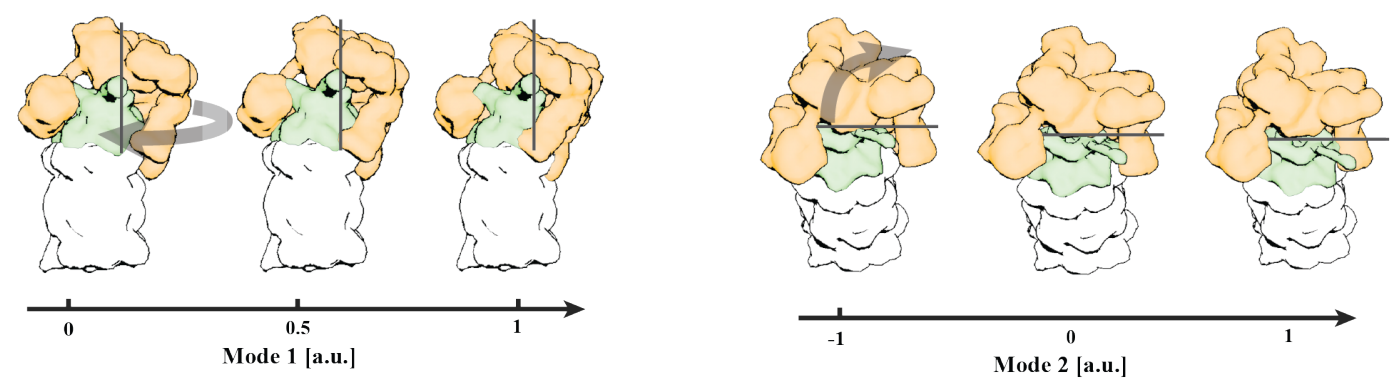

Figure 3.25: Energy landscapes and graphic representation of the modes. (A) Shows two energy landscapes, one of the non-inhibited and one of the Oprozomib-inhibited particles, respectively. Blue areas represent many particles and low energy, red areas mark areas with less particles and higher energy. The dark blue area in the right plot represents all particles belonging to the high resolution $3.8 \AA$ structure, found in a local energy minimum. In (2) the complex movement modes of the proteasome lid are represented in a graphical approach. Parts colored in orange belong to the regulatory particle, green represents all parts of the ATPase. The arrow indicates the direction of the main movement. The gray lines help to distinguish between the 3 main conformational stage. Figure adapted from Haselbach et al., $2017^{146}$. 


\section{Chapter 4}

\section{Discussion}

In this thesis crystallographic analysis of the native human 20S proteasomes and seven inhibitor-20S proteasome complexes was performed and the structural impact of $20 \mathrm{~S}$ inhibitors on the proteasome holoenzyme was studied by single particle cryo-EM.

The results obtained provide an important contribution to the understanding of proteasome inhibition. The key achievements of this thesis can be summarized as follows:

1. Optimized and robust pipeline for the production, purification and crystallization of human $20 \mathrm{~S}$ proteasomes.

2. Native human $20 \mathrm{~S}$ proteasome crystal structure at $1.8 \AA$ resolution identifies solvent molecules and functionally important differences with respect to previously published data.

3. Crystal structures of four previously uncharacterized inhibitor complexes of the human 20S proteasome with clinically relevant inhibitors and determination of novel inhibition chemistry.

4. Cryo-EM structure determination of the inhibited and non-inhibited human $26 \mathrm{~S}$ proteasome.

5. Identification of long-range allosteric regulation of the human $26 \mathrm{~S}$ proteasome by 20S core particle inhibitors. 


\subsection{Pipeline for Structure-based Analysis of Human Proteasome Inhibition}

The successful crystallographic and electron cryomicroscopic studies rely on a high level of reproducibility in generating proteasomes of high purity and abundance. Sample homogeneity should allow high-quality electron cryomicroscopic analysis and a reproducible crystallization. Hence, great efforts were undertaken to develop a purification procedure for both, human 20S and 26S proteasomes.

Purification of proteasomes from various eukaryotic sources thus far rely on chromatographybased protocols. In this thesis, a generic procedure has been introduced, which overcomes the disadvantages of chromatographic purification, such as high costs and the lack of transferability to proteasomes from different sources. This new method allows the purification of proteasomes from different tissues using the same protocol of PEG fractionation and density gradient centrifugation. The presented low-cost, chromatography-free method results in a high yield of highly soluble and stable complexes. This gentle purification formed the basis for structural analysis of proteasome-inhibitor complexes presented in this thesis and is transferable to a multitude of macromolecular complexes from all organisms.

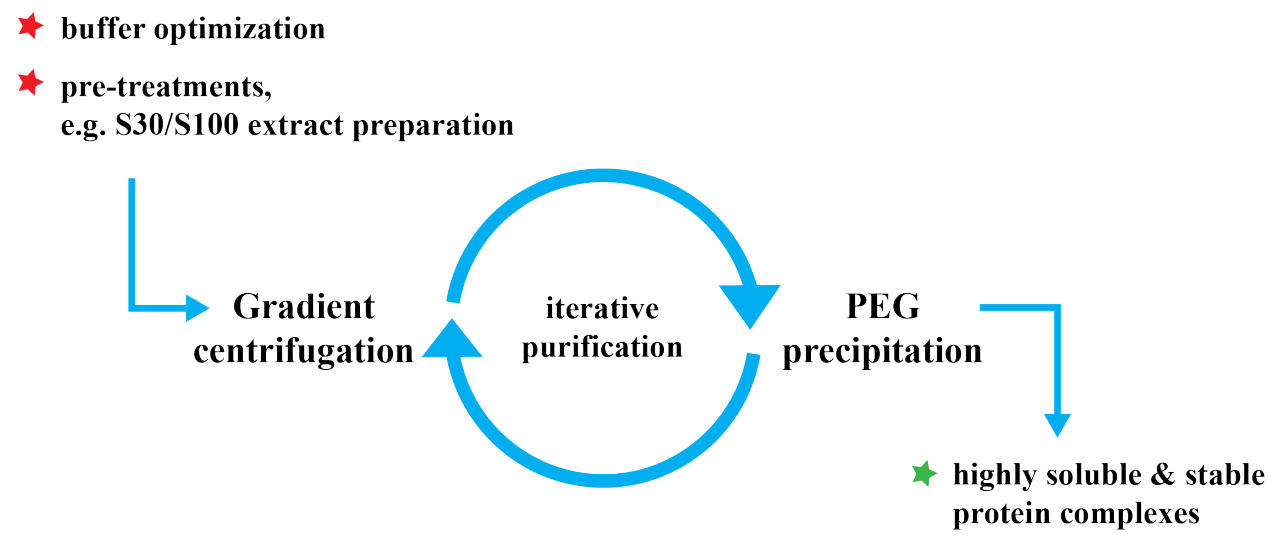

Figure 4.1: A chromatography-free purification procedure at constant ionic strength. PEG precipitation and gradient centrifugation is repeated iteratively until the desired purity/homogeneity is reached. Iterations may differ in PEG concentration and/or gradient composition/density. The method is adaptable to many protein complexes from all organisms.

The choice of a buffer system was a critical step in developing a method to purify functional human 20S and 26S proteasomes. In cells the composition of the fluid surrounding all proteins is very complex. In the laboratory one has to define the best reduced composition of a medium in which the protein is most stable. This medium is mostly a buffer substance supplemented with a mixture of salts. Proteins behave differently in varying buffer and $\mathrm{pH}$ conditions and every molecule has its own favored buffer composition. 
A generic method that allows to search for the most stabilizing buffer and $\mathrm{pH}$ condition was developed by colleagues ${ }^{148}$. The chromatography-free purified human $20 \mathrm{~S}$ proteasome sample showed its most stable condition in the buffer substance Bis-Tris at a $\mathrm{pH}$ of 6.5. This correlates with the physiological pH of intracellular fluids between 6.5 and $7.5^{149}$. All previously published 20S purifications were performed in Tris-HCl buffer at a $\mathrm{pH}$ of $7.5^{150,25}$.

Very stable and durable protein complexes were purified utilizing the gentle purification procedure and the most stabilizing buffer-pH combination. The proteolytic specific activity is an important indication for the proteasome's quality. The chromatography-free purified human $20 \mathrm{~S}$ proteasomes has a specific activity that is by a factor 10 higher than reported for previous human $20 \mathrm{~S}$ proteasomes ${ }^{22}$. 


\subsection{Native Human 20S Proteasomes Structure at $1.8 \AA$ Resolution}

Previously known crystallization conditions for 20S proteasomes require high protein concentrations. For yeast proteasomes the concentration for successful crystallizations lie in the range of 30 to $40 \mathrm{mg} / \mathrm{ml}$. We found a new crystallization condition for the human $20 \mathrm{~S}$ proteasome. Human 20S proteasomes obtained from chromatography-free purification crystallized in a crystal form not yet described for $20 \mathrm{~S}$ proteasomes. The optimum protein concentration is reproducibly at $7.5 \mathrm{mg} / \mathrm{ml}$ and a drop size of $1 \mu \mathrm{l}$ (1:1 mixture with mother liquor). With this comparatively small amount of protein, screening of many different crystallization conditions and protein-ligand complexes is possible on a large scale.

Human 20S proteasome crystals needed to be stabilized and dehydrated in a controlled manner. Complete isotropic diffraction data sets to $1.8 \AA$ resolution were collected, utilizing a complex postcrystallization protocol. For human 20S proteasomes the crystallization and postcrystallization buffer is identical to the purification buffer (Bis-Tris pH 6.5).

The high-resolution human $20 \mathrm{~S}$ proteasome structure at $1.8 \AA$ resolution reveals a number of functionally important differences of the $20 \mathrm{~S}$ proteasome active sites with respect to previously published 20S structures. A chloride ion was identified in all three catalytic sites and it was shown that this solvent molecule acts as a proton shuttle. In previous structural studies a water molecule was assigned in the chloride position ${ }^{16,25,143,151}$. This water molecule was described in the yeast $20 \mathrm{~S}$ proteasome as an ideally positioned solvent nucleophile involved in both intramolecular autolysis and substrate proteolysis by mediating proton transfer between $\mathrm{Thr} 1 \mathrm{O} \gamma$ and $\mathrm{Thr} 1 \mathrm{~N}^{16,25}$. Since the solvent molecule in this position is easily replaced as shown by several experiments it is unlikely that this solvent molecule acts as a nucleophile.

In addition, three localized water molecules were identified in the high quality electron density of the human 20S proteasome catalytic site. These water molecules were not previously described and the possible role of these solvent molecules in the active site of proteasomes was addressed in further experiments.

The possibility to describe the active site of the human $20 \mathrm{~S}$ proteasome at an unprecedented resolution opens up the opportunity to investigate ligand binding in the active sites at a whole new level. With structures at $1.8 \AA$ resolution the information content of the electron density map increases 3-fold compared to the previously published data. The resolution of the previously published $20 \mathrm{~S}$ structures from $S$. cerevisiae would allow for identification of the ligands in the active sites but the data are severely anisotropic that results in poor electron density maps ${ }^{16,152,153}$. X-ray data from higher organisms was collected at relatively low resolution which makes it impossible to obtain a detailed view 
of the ligands and solvent molecules in the active site ${ }^{23,22,44,25}$.

With the availability of the excellent model for the native human $20 \mathrm{~S}$ proteasome, now structure determination of $20 \mathrm{~S}$ proteasome structures can occur within minutes by automated refinement. Ligands can then be rapidly identified and modeled. 


\subsection{Human 20S Proteasome Inhibition}

In recent years, several co-crystal structures of the yeast $20 \mathrm{~S}$ proteasome in complex with inhibitors were elucidated at resolutions between 2.5 and $3 \AA$. These structures were used to describe the mechanism of proteasome inhibition ${ }^{70,25,73}$. The electron density in the active site of the $20 \mathrm{~S}$ proteasomes was of insufficient resolution and quality to model the inhibited state in atomic detail. Binding mechanisms of epoxyketone, ketoaldehyde and boronic acid inhibitors were proposed based on these co-crystal structures ${ }^{70,73,152}$.

In this thesis, crystallographic analysis of seven co-crystal structures of the human $20 \mathrm{~S}$ proteasome with cancer therapeutics was performed. The crystal structures of human proteasome-inhibitor complexes were elucidated at improved resolutions between 1.8 and $2.2 \AA$. The inhibitors adopt antiparallel $\beta$ sheets in the active sites of the human $20 \mathrm{~S}$ proteasome. These $\beta$ sheets are stabilized by direct hydrogen bonds between the conserved residues of the $\beta$-type subunits and main chain atoms of the ligand. The excellent electron density provides insight into the inhibited active site at an atomic level as never seen before, and new information about the binding mechanisms and the role of localized solvent molecules was identified.

\section{Epoxyketone Inhibitors}

A mechanism for epoxyketone inhibition was presented long before this study started. The inhibition was decribed as an irreversible reaction of the N-terminal amino group of the Thr1 with the $\alpha$-carbon atom of the epoxide, resulting in a 6 -membered 1,4-morpholine ring closure ${ }^{70}$. The observations leading to this hypothesis were made in yeast $20 \mathrm{~S}$ proteasomes inhibited by the epoxyketone inhibitor Epoxomicin at a resolution of $2.25 \AA^{70}$. Three co-crystal structures of human epoxyketone-20S complexes were analyzed and a revised description of epoxyketone inhibition was made. The crystal structures of epoxyketone inhibited human $20 \mathrm{~S}$ proteasomes show a density for an additional atom in the binding site. This identified the formation of a 7-membered 1,4-oxazepane ring structure upon inhibitor binding. This observation was confirmed by theoretical simulations and a series of control experiments. Hence, these findings allow a novel chemical mechanism for the inhibition of 20S proteasomes by epoxyketone inhibitors to be proposed (Figure 4.2): The $\gamma$-hydroxyl group of the Thr1 of the proteasome active site reacts with the ketone moiety of the epoxyketone inhibitor, as described for the predicted 1,4-morpholine ring formation. The N-terminal amino group of Thr1 then reacts with the carbon atom of the epoxide that is in $\beta$ position to the ketone. The carbon atom of the epoxide in $\beta$ position is less hindered for a reaction with the N-terminal amino group of Thr1. 
A

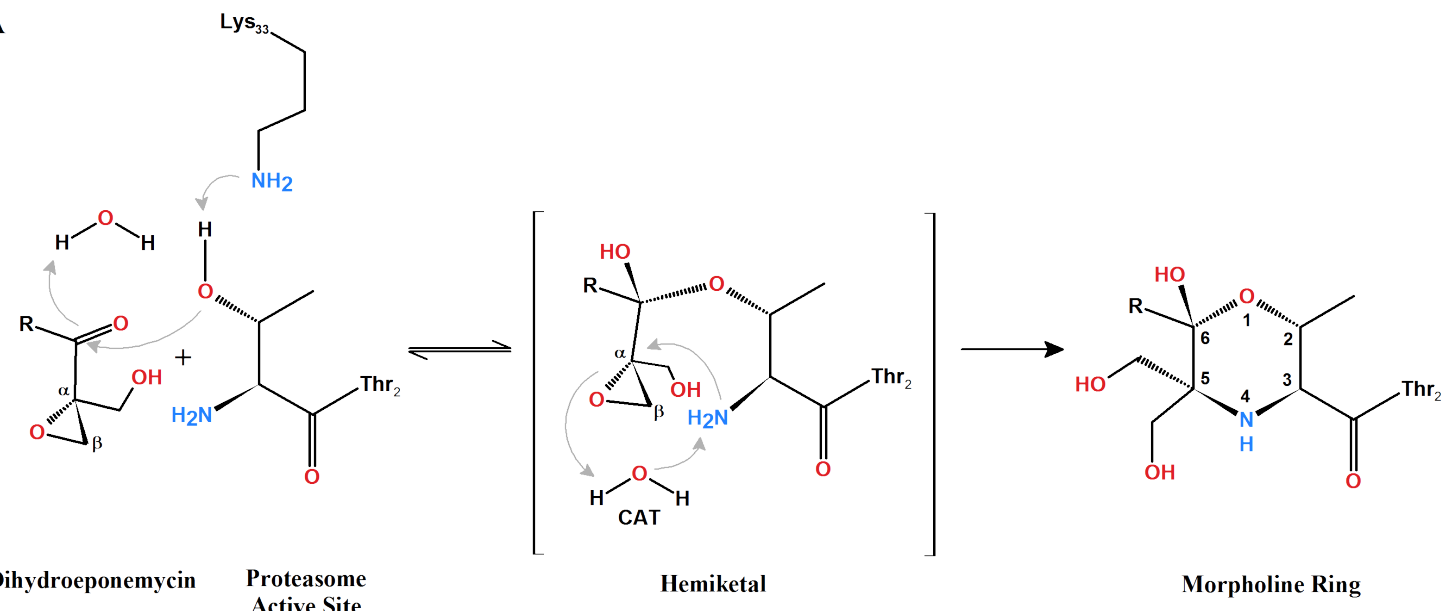

B<smiles>CCCCNCNOC(C)C(N)C(=O)[NH2+]C</smiles>

Dihydroeponemycin
Proteasome Active Site

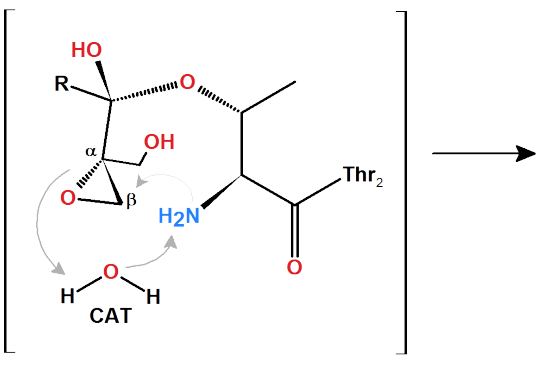

Hemiketal<smiles>CC(=O)C1NCC(CO)(CO)C(O)(O)OC1C</smiles>

Oxazepane Ring

Figure 4.2: Comparison of 6- and 7- ring forming reaction mechanisms for Dihydroeponemycin. (A) The reaction mechanism for epoxyketone inhibitors proposed thus far using the example inhibitor Dihydroeponemycin. Groll et al. ${ }^{70}$ proposed that the Thr1-O $\curlyvee$ initially reacts with the ketone to form a hemiketal (middle). The N-terminal amine of Thr1 then reacts with the epoxide $\alpha$-carbon atom resulting in a 1,4-morpholine linkage. (B) The novel inhibition mechanism which leads to the formation of a 7-membered 1,4-oxazepane linkage. Here, the Thr1 N-terminal amine is involved in a nucleophilic reaction with the epoxide $\beta$-carbon atom.

Since it is difficult for crystallographers to interpret whether only one binding mechanism occurs or if a mix of two structures is present, a multitude of control experiments were carried out. The results showed unequivocal evidence that the electron density allows only the 7-ring formation in the human 20S proteasome.

Researchers previously describing the inhibition mechanism, suggested the 6-ring formation based on Baldwin's rules ${ }^{70}$. Their observation of chemical reactivities in the Epoxomicin inhibited yeast 20S proteasome lead to the assumption that the 6-ring is formed by a exo-tet process, whereby the 7-ring is formed by an endo-tet process. Exo-tet is favored over endo-tet. It was stated that the 6-ring exo-tet is the favored and therefore prominent mode of epoxyketone inhibition.

The chemical rules defined by Baldwin were incorrectly applied to the epoxyketone 
inhibition by Groll et al. ${ }^{70}$. In short, the Baldwin rules provide the terminology needed to discuss cyclization reactions, allow a classification of cyclization, and suggest a set of rules to define the favorable modes of ring closure ${ }^{154}$. Cyclization described by the Baldwin rules is characterized by three prefixes. The first one provides the number of atoms forming the ring, starting at a value of three. The second prefix describes the position of the bond that has to be broken for cyclization of the smallest ring in the structure: exo and endo. Exo means that the breaking bond is outside of the formed ring, whereas endo describes that the breaking of the bond happens inside the ring. Lastly, the prefix tet (tetrahedral), - trig (trigonal), and - dig (diagonal) refers to the hybridization of the atom at the site of the ring closure. With greater understanding based on thousands of examples, a number of extensions and revisions of the Baldwin rules have arisen. One extension was introduced for 3-membered ring structures, the epoxides. Cyclization that involves a nucleophilic epoxide ring opening should not be termed endo-tet. The use of both, exo-tet and endo-tet for epoxide closures is stated as misconception. The breaking epoxide $\mathrm{C}-\mathrm{O}$ bond is located outside the newly formed ring and each cyclization should be considered exo-tet ${ }^{155,156}$. That means all epoxyketone ring-formations have to be classified exo-tet. Here, epoxyketone inhibitors bind in a 7-exo-tet process. By the Baldwin rules the 7-exo-tet process is not favored or unfavored over the 6-exo-tet ring closure. Hence, this argument was neglected in our studies.

We utilized epoxyketone inhibitors because they are the most advanced and specific proteasome inhibitors known to date. With an irreversible binding mode and no known site effects to date is this class of inhibitors desirable as cancer therapeutic. Utilizing the robust pipeline to obtain structural information at higher resolution the atomic details of the epoxyketone inhibition mechanism were shown. For epoxyketones it was outlined that only one of the two electrophilic groups of the epoxide is involved in the inhibition. Here, only the carbon in the $\beta$-position to the ketone reacts with the N-terminal Thr1 of the proteasomal active site. Based on the novel inhibition chemistry, there are several possible options for the design of new irreversible proteasome inhibitors exhibiting dual electrophilic head groups. 


\section{Ketoaldehyde Inhibitors}

To explore the structural implications of a "true" 6-ring linkage, the ketoaldehyde inhibitor Z-LLY-Ketoaldehyde was subjected to structural analysis. Like epoxyketones, ketoaldehyde inhibitors take specific advantage of the unique catalytic mechanism employed by the proteasome. The inhibition mechanism for ketoaldehydes proceeds only via a carbon atom in the $\alpha$-position of the ketone with the Thr1 of the $20 \mathrm{~S}$ proteasome active site.

The crystal structure of the Z-LLY-Ketoaldehyde-20S complex was determined at $2.1 \AA$ resolution with high quality electron density in the active sites. The concept of ketoaldehyde binding in the yeast $20 \mathrm{~S}$ proteasome was published five years ago ${ }^{73}$. Interpretations of the actives site's electron density at $2.7 \AA$ lead to the assumption that the inhibitor forms a 6 -membered heterocyclic, 5,6-dihydro-2H-1,4-oxazine ring ${ }^{73}$. Ketoaldehydes harbor a reversible binding mechanism, due to the Schiff base. Unexpectedly, the binding mechanism for ketoaldehyde inhibitors was misinterpreted.

Our $2.1 \AA$ resolution data confirm a 6-ring formation, but unlike the heterocyclic ring closure, the electron density reveals a rigid 6-ring morpholine linkage. This indicates that a water molecule is not released from the carbinolamine. The morpholine ring that was predicted to be a transition state is the final product of this inhibition (Figure 4.3). This observation could only be made because of excellent electron density at the site of inhibition.

Further, the high resolution structure of the Z-LLY-Ketoaldehyde inhibited human 20S proteasome allowed to identify that the $\mathrm{H}_{2} \mathrm{O}-3$ molecule is involved in the hydrogen-bond network of the $\mathrm{C} 5-\mathrm{OH}$ moiety. This implicated a putative role of this water in the binding mechanism of inhibitors which was confirmed in later inhibition studies with boronic acid inhibitors. 
A

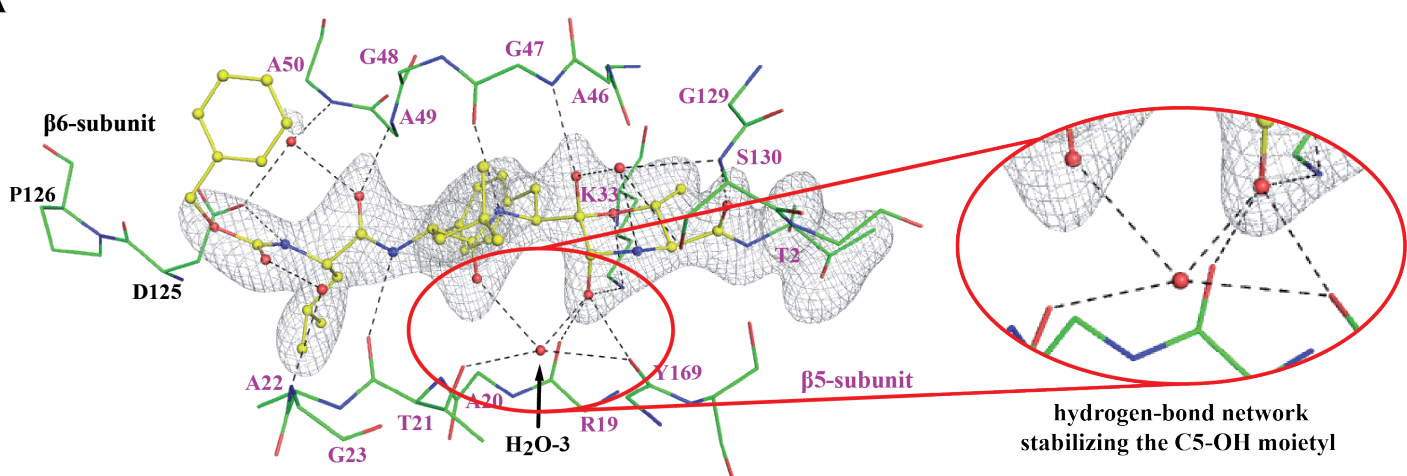

B

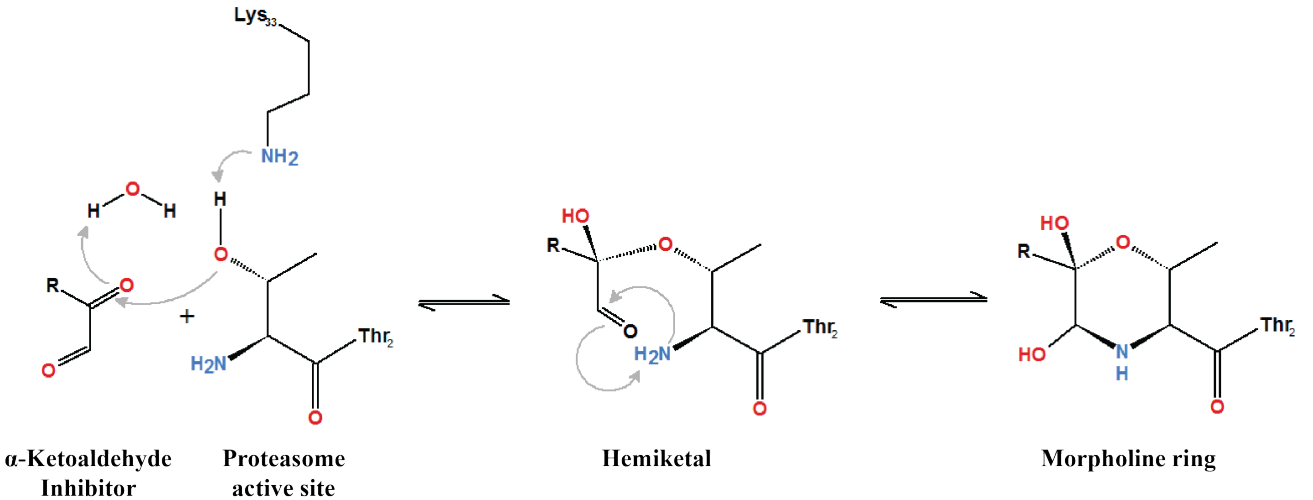

Figure 4.3: Human 20S proteasomes inhibited by Z-LLY-Ketoaldehyde. (A) Hydrogen network of the C5-OH moiety. The $\mathrm{H}_{2} \mathrm{O}-3$ molecule is identified to be involved in the stabilization. (B) Proposed inhibition mechanism for $\alpha$-Ketoaldehydes. The electron density in the active site shows a six-membered ring with a clear density for a hydroxyl group at the C5 position of the linkage. Z-LLY-Ketoaldehyde inhibition results in a 1,4-morpholine linkage. 


\section{Boronic Acid Inhibitors}

The binding mode of boronic acid inhibitors mimics the first tetrahedral intermediate state in substrate proteolysis. Boronic acid inhibition was analyzed to address the putative role of the $\mathrm{H}_{2} \mathrm{O}-3$ water molecule in the active site.

Boronic acid inhibitors Bortezomib, Ixazomib and Delanzomib were chosen for X-ray analysis. The organization in the Bortezomib inhibited chymotryptic-like site of yeast $20 \mathrm{~S}$ proteasome was analyzed previously at $2.8 \AA$ resolution ${ }^{152}$ : The boron atom of the tetrahedral boronate adduct interacts covalently with the Thr1 $\mathrm{O} \gamma$. The strictly conserved oxyanion hole Gly47N is hydrogen-bridged to one of the acidic boronate hydroxyl groups. The tetrahedral boronate adduct is stabilized by an additional hydrogen-bridge of a second hydroxyl moiety with the N-terminal Thr1 amine atom.

We could evaluate and redefine the critical interactions of boronic acid inhibitors with the human 20S proteasome active site. Boronic acid-20S proteasome complex structures were determined at 2.0 and $2.1 \AA$ resolution. We found that the $\mathrm{H}_{2} \mathrm{O}-3$ stays in a prominent position upon inhibitor binding in orientation to support the cyclization step of ringforming inhibitors (Figure 4.4). $\mathrm{H}_{2} \mathrm{O}-1$ and $\mathrm{H}_{2} \mathrm{O}-2$ are displaced from the active site upon inhibitor binding. $\mathrm{H}_{2} \mathrm{O}-3$ is hydrogen-bonded to one hydroxyl group of the boronic acid moiety, stabilizing the tetrahedral state. From computed pathways and on the basis of the inhibitor-20S proteasome structures we propose that the $\mathrm{H}_{2} \mathrm{O}-3$ most likely serves as the decisive factor in the cyclization step in ketoaldehyde and epoxyketone inhibition. The significance of these findings might allow for a better understanding of the catalytic details of inhibition and additional inhibitor design.

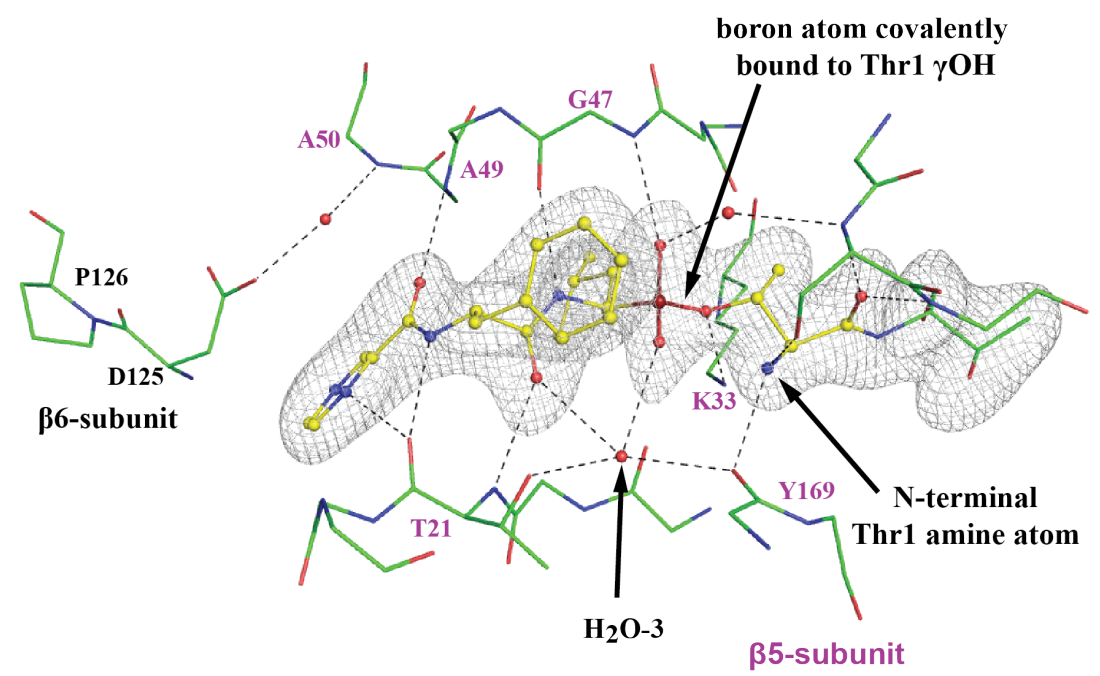

Figure 4.4: Critical interactions of Bortezomib in the active site of human 20S proteasomes. Proposed interactions of boronic acid inhibitors in the human 20S proteasome catalytic site. The boron atom is covalently linked to the Thr1 $\mathrm{O} \gamma$. Gly47N and $\mathrm{H}_{2} \mathrm{O}-3$ stabilize the tetrahedral boronate adduct with hydrogen-bridges. No hydrogen bond formation between the N-terminal Thr1 amine atom and the acidic boronate hydroxyl moiety could be visualized. 


\subsection{Structural Impact of 20S Inhibitors on the 26S Holoenzyme}

Knowledge about the structural impact of core particle inhibitors on the human 26S proteasome holoenzyme might provide insight into the proteasome's mechanism of operation. Furthermore, this might serve as a platform for inhibitor screening and the development of future proteasome inhibitors.

The capacity of the proteasome to degrade proteins substantially depends on the core particle-regulatory particle interaction. It was previously shown that proteasome inhibitors are potent in proteasome stabilization ${ }^{145}$. In 2007, Finley and colleagues could show by in vitro reconstitution assays that epoxyketone and boronic acid inhibitors stabilize the CP-RP interaction. These findings suggested that core particle inhibition is communicated to the interface between core particle and regulatory particle. They envisioned that proteasome inhibition is carried out by an allosteric mechanism. The flow of information is directed from the active site deep in the $20 \mathrm{~S}$ proteasome towards the regulatory particle ${ }^{145}$.

Allosteric regulation was later discovered in the yeast and mouse $20 \mathrm{~S}$ proteasome ${ }^{157}$. Conformational changes in the protease become visible upon peptidic ligand binding at the $\beta 5$ active site. Comparison of all 28 subunits of the unliganded $20 \mathrm{~S}$ proteasome with a peptide-20S proteasome complex demonstrated an enhanced plasticity of the $\beta 5$ subunit. This suggests specific signaling pathways to other subunits. The tight packing of the subunits in the CP together with the observed conformational changes opened the possibility of signal propagation from $\beta 5$ to other subunits. Most of the conformational alterations in the $\beta 5$ subunit induced by the peptidic binding were observed distant from the active site's Thr1 and located at the surface of the $20 \mathrm{~S}$ proteasome. In yeast $20 \mathrm{~S}$ proteasomes, the binding of a peptidic ligand causes domain closure and movements of about $1 \AA^{157}$. Still, communication between the 20 S core particle and the $19 \mathrm{~S}$ regulatory particle is unexpected because of the large distance, but movement in the subunits of the $20 \mathrm{~S}$ core particle seems to propagate towards the surface of the 19S regulatory particle. In the yeast $26 \mathrm{~S}$ proteasome different conformations were previously observed in electron cryomicroscopic studies. Under native in vitro conditions approximately $80 \%$ of the yeast proteasome particles were found in the relaxed, non-rotated conformational state (Figure 4.5$)^{158,51}$. Binding of a slowly degraded substrate to the regulatory particle ${ }^{158}$ or treatment with the slowly-hydrolyzable nucleotide ATP $\mathrm{AS}$ has a direct effect on the conformational state of the $26 \mathrm{~S}$ proteasome. The rotated conformational state is then favored over the non-rotated state by $55 \%$ and $73 \%$, respectively (Figure 4.5$)^{51}$. Sledz et al. proposed that the yeast $26 \mathrm{~S}$ proteasome switches to a actively translocating state upon substrate degradation: The AAA + ATPase becomes coaxially aligned to the core 
particle pore, the ubiquitin receptor Rpn10 contacts the Rpt4-Rpt5 coiled coil and the DUB Rpn11 shifts to a central position directly above the N-ring pore of the AAA+ ATPase. These structural rearrangements are similar to the $26 \mathrm{~S}$ proteasome particles found in the rotated state in the presence of ATP $\gamma \mathrm{S}$.
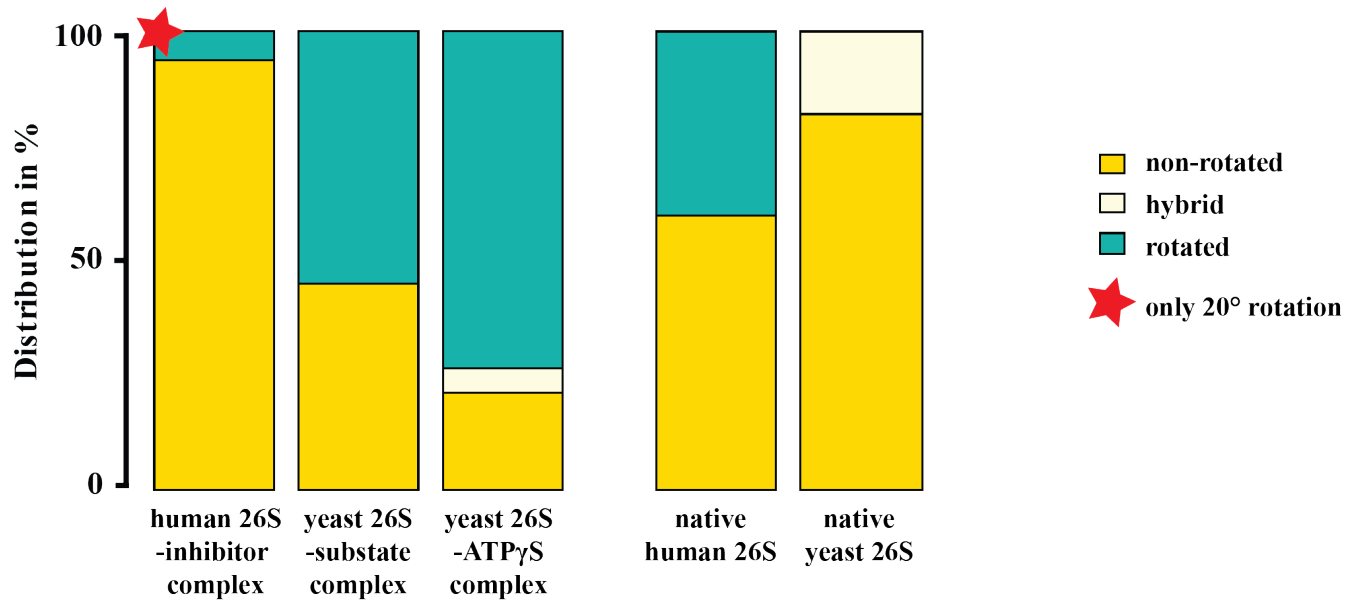

Figure 4.5: Relative frequencies of non-rotated, hybrid and rotated state. Comparison of relative frequencies of the three conformational states of the regulatory particle. Left to right: Human 26S proteasomes in complex with Oprozomib, yeast 26S proteasomes with substrate bound to the 19S subunit, yeast $26 \mathrm{~S}$ proteasomes in presence of $\mathrm{ATP} \gamma \mathrm{S}$, native human $26 \mathrm{~S}$ proteasomes, native yeast proteasomes ${ }^{51,158}$. Human $26 \mathrm{~S}$ proteasomes inhibited by Oprozomib are predominantly found in the non-rotated state $(94 \%)$. The remaining particles are found in a rotated state, whereby the main lid rotation is limited to $20^{\circ}$.

When Finley and colleagues formulated the first hypothesis about allosteric regulation of the $26 \mathrm{~S}$ proteasome by $20 \mathrm{~S}$ inhibitors they did not have the possibilities to investigate this structurally. We are now able to determine structures of the $26 \mathrm{~S}$ proteasome holoenzyme with and without epoxyketone inhibitors by single particle cryo-EM.

We identified a clear restriction of the conformational landscape of the human 26S proteasome upon inhibitor treatment, while the overall conformation of the non-inhibited and inhibited form is identical. Previous studies showed that treatment of $26 \mathrm{~S}$ proteasomes with $20 \mathrm{~S}$ proteasome inhibitors leads to a stabilization of polyubiquitinated substrates ${ }^{147}$. We found no evidence for a profound accumulation of polyubiquitinated substrates in the inhibited sample by western blot analysis. With this we could exclude that the structural differences derive from polyubiquitinated substrates attached to the $26 \mathrm{~S}$ proteasome.

We proceeded then to analyze the Oprozomib-inhibited and the non-inhibited $26 \mathrm{~S}$ proteasome by cryo-EM. This resulted in a final map of the Oprozomib-inhibited human $26 \mathrm{~S}$ proteasome at a resolution of $3.8 \AA$ and the non-inhibited at $4.8 \AA$. We found that inhibitor binding induces an energy barrier minimizing the possibility of the regulatory particle to rotate on the core particle. This is reflected by the higher resolution structure of the Oprozomib-inhibited 26S proteasome compared to the non-inhibited structure. $12 \%$ of all particles of the Oprozomib dataset contributed to the high resolution Oprozomib- 
$26 \mathrm{~S}$ proteasome complex. Only $4 \%$ of all particles of the native dataset are found in the lower resolution reconstruction of the non-inhibited $26 \mathrm{~S}$ proteasome.

Inhibition of the human $26 \mathrm{~S}$ proteasome by $20 \mathrm{~S}$ core particle inhibitors leads to a stabilization of the proteasome holoenzyme in the non-rotated state (Figure 4.5). Thus, inhibitor binding in the core particle of the holoenzyme shows a distinct opposing effect on the conformational landscape than substrate binding to the regulatory particle or blockage of the AAA+ ATPase. Regulation of the conformational motion seems to occur from two directions: the $19 \mathrm{~S}$ regulatory particle or the $20 \mathrm{~S}$ core particle, resulting in opposing conformational states. In Figure 4.5 the relative frequencies of all previously described prominent conformational states in different proteasome preparations are shown. The native yeast conformation distribution differs from the native human distribution. In yeast, most particles are found in the non-rotated state $(82 \%)$ and the remaining particle are found in a hybrid state (S2). Native human 26S proteasomes have an almost even distribution between non-rotated and rotated states (60\% to $40 \%$ ).

The same trend was observed from in situ studies of mammalian hippocampal neurons ${ }^{159} .80 \%$ of the $26 \mathrm{~S}$ proteasome found in the cell were in the ground state and $20 \%$ in the rotated substrate processing state. This correlates well with the native human $26 \mathrm{~S}$ proteasome dataset. In addition, we performed $26 \mathrm{~S}$ proteasome data analysis of three independent proteasome preparations. Classification of the datasets was performed carefully and in an exemplary way to allow for statistical analysis.

In conclusion, our data show that $20 \mathrm{~S}$ core particle inhibition shifts the $26 \mathrm{~S}$ particle distribution towards the non-rotated state. In contrast, $26 \mathrm{~S}$ proteasomes in complex with substrates in the $19 \mathrm{~S}$ regulatory particle or in the presence of ATP $\mathrm{AS}$ have a higher fraction of particles in the rotated conformational state. This points to the fact that the $26 \mathrm{~S}$ proteasome can be manipulated at various sites and we propose a bi-directional signaling pathway throughout the $26 \mathrm{~S}$ proteasome. This is presented and discussed in the following section. 


\subsection{A Potential bi-directional Signaling Pathway}

Electron cryomicroscopic analysis of inhibited human 26S proteasomes provides evidence that inhibition of the $20 \mathrm{~S}$ proteasome allosterically regulates the conformation of the holoenzyme. The inhibitor bound to the proteolytic site in the core particle has the possibility to send a signal to the regulatory particle which is converted and amplified into structural changes. Earlier studies presented a similar allosteric effect mediated from the regulatory particle towards the 20S core particle.

To address the question of which determinants might be involved in the long-range communication throughout the proteasome holoenzyme, a detailed investigation of the dynamics of distinct parts of the proteasome was carried out. Two important and correlated criteria to investigate conformational mobility were taken into account: decreased local resolutions in the EM map and regions with high B-factors in the atomic model.

The chemical signal of inhibition is located in the $\beta 5$ subunit of the $20 \mathrm{~S}$ core particle and is propagated towards the outer parts of the regulatory particle. Starting from the site of inhibition, a hypothesis for propagation of signals throughout the holoenzyme is presented.

\section{The $\alpha$-ring}

The upper $\alpha$-ring is located between the inhibitor binding site and regulatory particle. This $\alpha$-ring serves as a platform for regulatory particle binding to the $20 \mathrm{~S}$ core particle. A gradient of flexibility within the $\alpha$-ring becomes visible. A significant increase in B-factors was found on the outer parts of the $\alpha$-ring subunits $\alpha 2, \alpha 3$ and $\alpha 4$, as shown in Figure 4.6. These subunits are located above the Oprozomib binding site in the $\beta 5$ subunit.

The asymmetric B-factor elevation of the $\alpha$-ring might directly be relayed to the regulatory particle subunits Rpn5 and Rpn6. The N-termini of Rpn5 and Rpn6 are extended towards the $\alpha 1$ and $\alpha 2$ subunits of the 20S particle. The atomic model of these N-terminal domains revealed high B-factors (Figure 4.6). A direct lateral transmission of the inhibitory signal onto the regulatory particle Rpn5 and Rpn6 subunits is possible according to these data.

\section{The AAA + ATPase}

The next area which shows conformational mobility is the next higher level in vertical transmission: the AAA + ATPase. The AAA + ATPase is part of the 19S regulatory particle and connects the core particle with the regulatory particle through binding of 
Figure 4.6: B-factors of the upper $\alpha$-ring. Model of the $\alpha$-ring together with the 19S subunit N-terminal domains of Rpn5 and Rpn6. Colored according to the B-factors. The HbXY motifs of Rpt3 and Rpt5 (green and cyan ball-and-stick model) are bound in the corresponding binding pockets. The orange arc line depicts the position of Rpt3 which shows the highest B-factor.
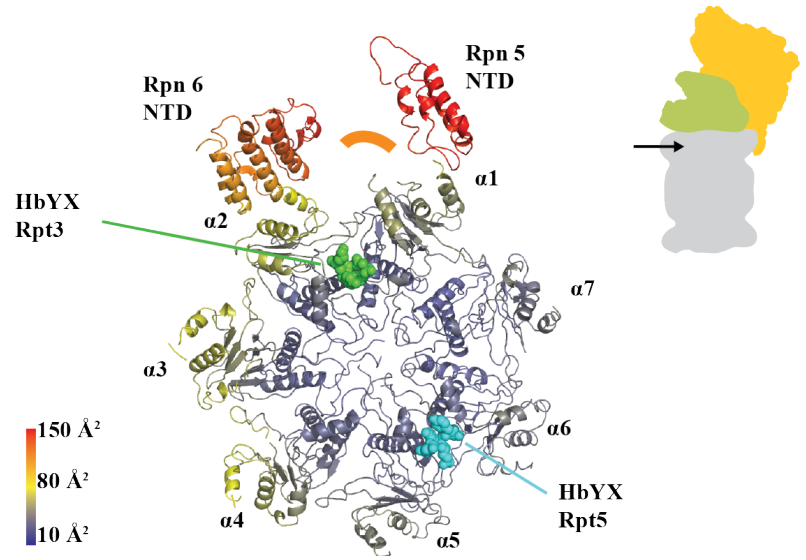

N-terminal tails of the HbYX motifs into the binding pockets of the $\alpha$-ring. The motifs of AAA + ATPase subunits Rpt3 and Rpt5 are bound to their corresponding binding pockets in the core particle (Figure 4.6). The density for the HbYX motif of Rpt2 is missing, indicating that the HbYX motif remains flexible. The AAA+ ATPase model with coloring according to the B-factors is shown in Figure 4.7. The subunits Rpt2, Rpt3 and Rpt6 show an increased mobility, represented by high B-factor values. These subunits are in close proximity to the highly flexible Rpn5 and Rpn6 N-terminal domains. Rpt2 is most proximal to the CP in the staircase arrangement of the ATPase. The unbound and flexible HbYX motif of Rpt2 might facilitate AAA+ ATPase motion on the 20S core particle.

The high flexibility on one side of the $\alpha$-ring is propagated to the AAA + ATPase subunits. The flexible parts are in close proximity to the N-terminal domains of Rpn5 and Rpn6 in both cases.

Figure 4.7: B-factors of the AAA + ATPase. Model of the AAA + ATPase together with the $19 \mathrm{~S}$ subunit N-terminal domains of Rpn5 and Rpn6. Colored according to the Bfactors. The B-factors increase towards the $\mathrm{AAA}+\mathrm{ATPase}$ parts which are adjacent to the N-terminal domains of Rpn5 and Rpn6. For better orientation, the position of the HbXY domains is indicated by blue asterisks.

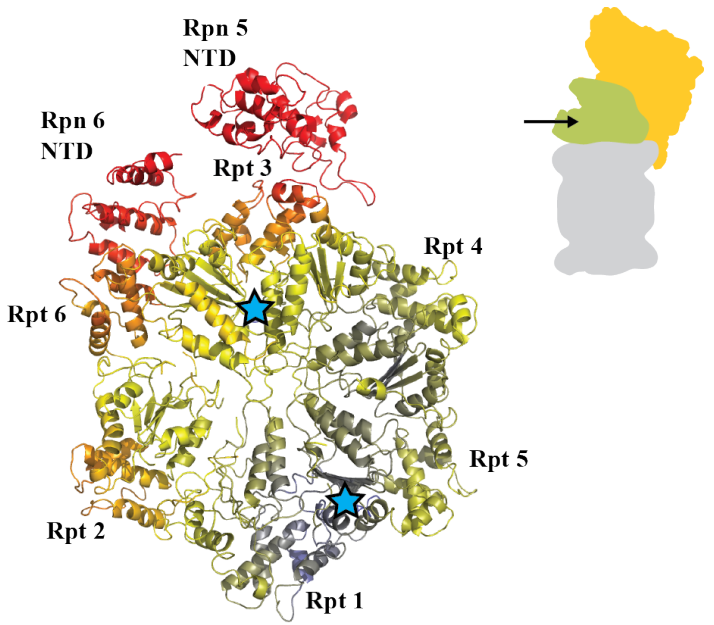

The symmetry of the dynamic AAA + ATPase was also investigated. The AAA+ ATPase rings deviate from a sixfold pseudosymmetry and the subunits are arranged in a helical staircase structure $^{31}$. For analysis, three conserved amino acids from all six ATPase subunits were chosen as anchor points. These amino acids are found in (a) the C-terminal region, (b) the middle part of the ATPase, and, (c) the N-terminal region near the center 
of the AAA+ ATPase. When connecting the $\mathrm{C} \alpha$-atoms of the conserved amino acids next to each other the resulting hexagon gives evidence of the angle of the symmetry of the $\mathrm{AAA}+$ ATPase. In Figure 4.8 the exact angles for the subunit Rpt2 are given. The Cterminal region is arranged in a perfect hexagon with an angle of $120^{\circ}$. The symmetry is distorted when measuring the N-terminal regions near the center of the AAA + ATPase. The angle measured for Rpt2 is decreased to $99^{\circ}$, indicating a vertical deviation from perfect six-fold symmetry. This is even visible in different sugar conformations of the bound nucleotide in subunit Rpt2 (Figure 4.9). The only nucleotide showing a different sugar pucker conformation is Rpt2. This might indicate major conformational changes in the areas around or the exchange to a different nucleotide in that particular position.
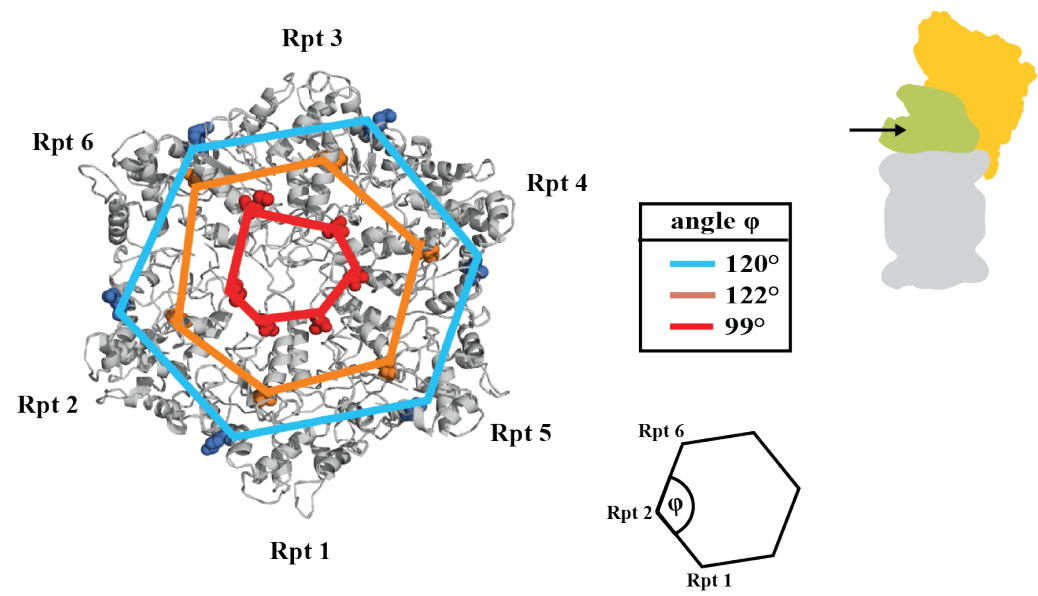

Figure 4.8: Deviation from perfect six-fold symmetry in the ATPase. The conserved amino acids are depicted in ball-and-stick representation. Hexagons were created by linking the aminoacids of the neighboring subunits. The C-terminal (outer) parts of the ATPase form a perfect hexagon. Moving towards the N-terminal region the hexagon becomes distorted. 

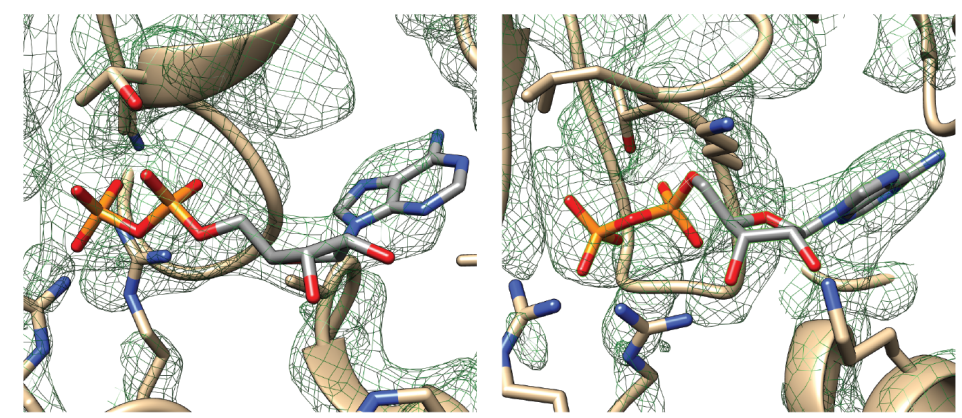

Rpt1

Rpt2
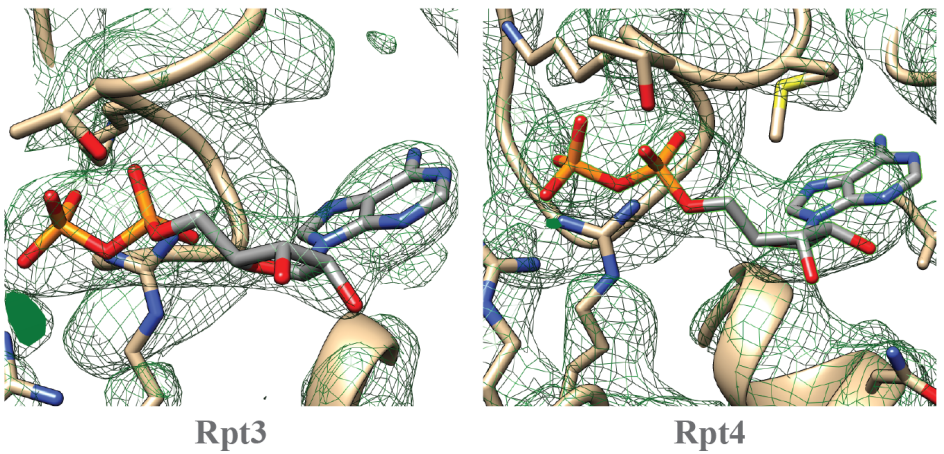

Rpt4
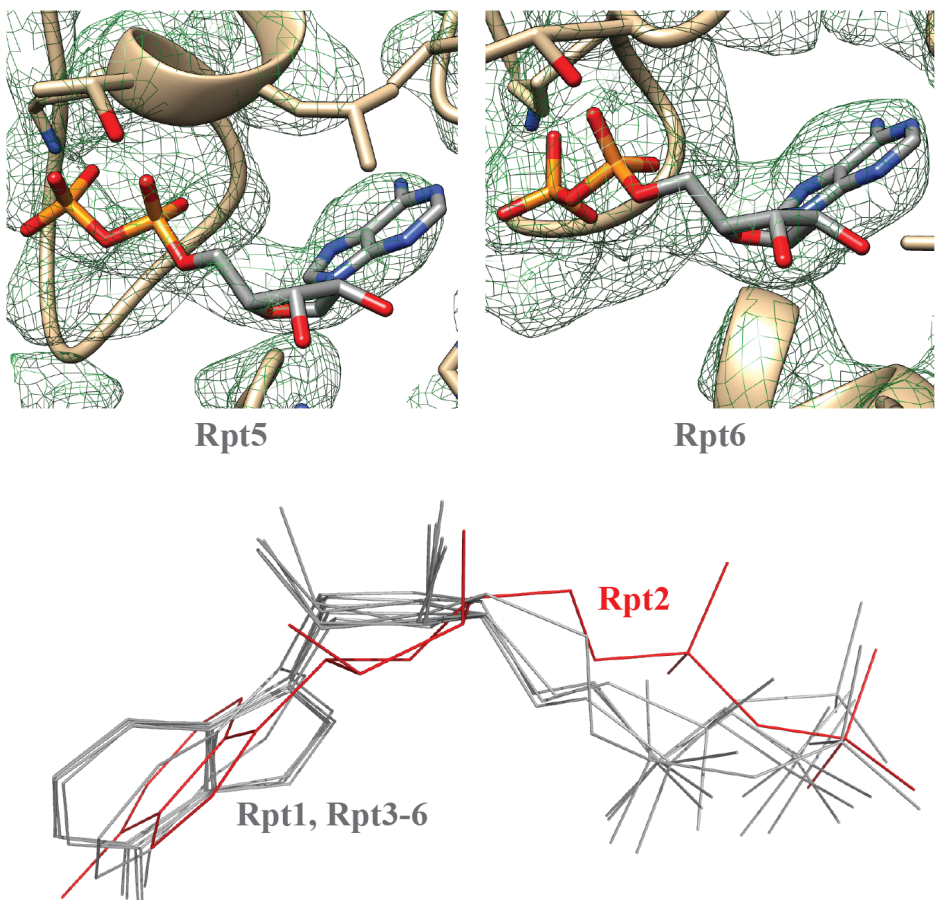

Figure 4.9: Different sugar conformation of nucleotide Rpt2. Close-up view of all six nucleotide densities in the AAA + ATPase of the human Oprozomib-26S proteasome complex. All nucleotides were built as ADP. Due to low resolution we could not distinguish between ADP and ATP nucleotides. The nucleotide bound to Rpt2 shows a different sugar pucker (bottom). 


\section{Rpn10 Domain Dynamics}

By focused classification on the ubiquitin receptor, Rpn10, a multitude of different conformations were found in the next higher level in vertical transmission. Figure 4.10 shows exemplary conformations of the highly flexible ubiquitin receptor, Rpn10. The receptor is located in the periphery of the regulatory particle above the ATPase domain and is in close proximity to Rpn5. The classification revealed conformers of Rpn10 in direct contact with the coiled-coil regions of the AAA+ ATPase domains Rpt4 and Rpt5. Other conformers of Rpn10 are completely detached from the adjacent subunit, the deubiquitinase Rpn8.

The proteasome subunit Rpn5 spans a distance of $100 \AA$ from the $20 \mathrm{~S}$ core particle passing the ATPase towards the ubiquitin receptor Rpn10. This makes Rpn5 a likely candidate to be involved in the propagation of the inhibitory signal from the active site in the core particle to the substrate receptor in the regulatory particles. Rpn5 is an essential lid protein, as described for yeast and plants ${ }^{160}$.
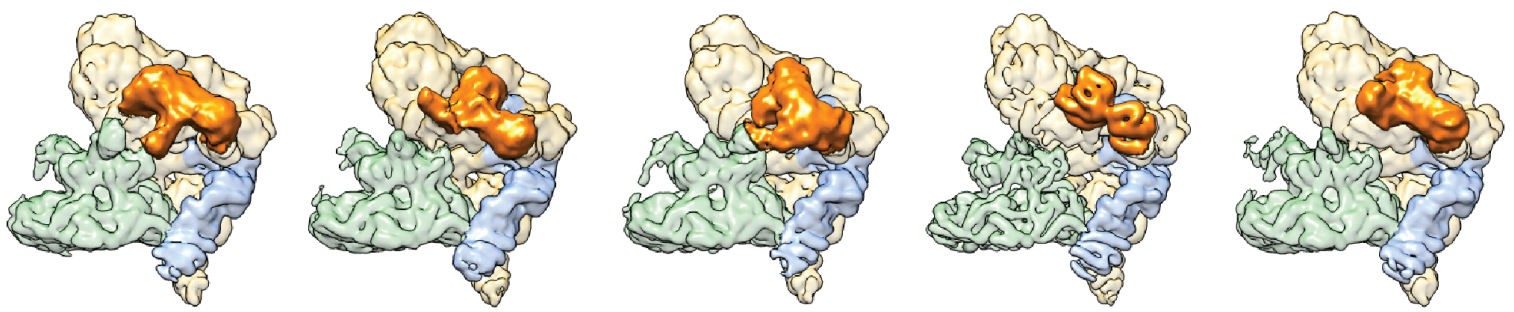

Figure 4.10: Conformational states of the Rpn10 protein. Focused classification on the ubiquitin receptor Rpn10 (orange) revealed a multiple conformational states. Exemplary conformational states are shown. The Rpn5 domain is colored in blue.

In conclusion, all sites of the $26 \mathrm{~S}$ proteasome that are in direct contact or in close proximity to Rpn5 show a high degree of flexibility and harbor the most mobile regions in the atomic model: The signal might be transmitted by Rpn5 from the site of inhibition in the core particle to the $\alpha$-subunit and the adjacent AAA + ATPase and come to a final stage by effecting the receptors of the regulatory particle. In general, the signal transmission seems to make it's way from deep in the barrel shaped 20S core particle to the surface of the $19 \mathrm{~S}$ regulatory particle. 


\section{Chapter 5}

\section{Conclusion and Outlook}

This work furthers the understanding of proteasome inhibition in the human 20S proteasome and gives first insights into structural influences of $20 \mathrm{~S}$ core particle inhibition on the 26S holoenzyme.

Firstly, a general workflow to gently purify endogenous human proteasome was established. High resolution crystal structures of the native human 20S proteasome and in complex with cancer therapeutics were analyzed. The native human $20 \mathrm{~S}$ proteasome structure was determined at $1.8 \AA$ resolution. This is the highest resolution yet attained for any proteasome structure. Collectively, the structures provide detailed insights into the active site and catalytic mechanisms of inhibition of the $20 \mathrm{~S}$ proteasome. The map details reveal substantial differences calling for a revised descriptions of the proteasomal active site. Co-crystal structures with inhibitors enabled the direct observation of novel inhibition mechanism that distinctly differ from earlier models derived from lower resolution maps.

The inhibition mechanisms will guide the design of next-generation proteasome-based cancer therapeutics. Our results suggest that peptide based inhibitors with dual-electrophile head groups would exhibit kinetically improved inhibition rates. With the workflow presented, we have a powerful and robust tool to structurally analyze hundreds of potential cancer therapeutics in complex with the human $20 \mathrm{~S}$ proteasome in a short time.

In the second part of this thesis, the effect of core particle inhibition on the human $26 \mathrm{~S}$ holoenzyme was analyzed by single particle cryo-EM. Since the human $26 \mathrm{~S}$ holoenzyme is an attractive target for the development of chemotherapeutics, the structure of the human 26S proteasome in complex with the 20S core particle inhibitor, Oprozomib was determined. We found that inhibitor binding modifies the conformational dynamics of the $19 \mathrm{~S}$ regulatory particle resulting in a stabilization of a non-productive state of the $26 \mathrm{~S}$ holoenzyme. We propose a mediation of the chemical drug-binding signal into structural changes over a distance of more than $150 \AA$. Furthermore, we present evidence that the regulatory particle subunit, Rpn5 most likely conveys the information from the catalytic center over this long-range distance. An allosteric effector that has such an extended reach was never described before. From previous publications it was known that substrate bind- 
ing to the regulatory particle also results in structural changes in the $26 \mathrm{~S}$ holoenzyme. Ligand binding to the regulatory particle has the opposing effect on the conformational landscape of the $26 \mathrm{~S}$ proteasome than ligand binding to the core particle. These findings indicate a bi-directional signaling pathway, mediated by a feedback regulation through regulatory particle subunit Rpn5. This knowledge allows a foresight: Core particle inhibitors with novel inhibition chemistry and inhibitors that target the regulatory particle might have a profound influence on the conformational variability and activity of the $26 \mathrm{~S}$ proteasome. Furthermore, the inhibitory signal is communicated to the surface of the $26 \mathrm{~S}$ proteasome and detectable by structural methods.

A detailed knowledge of the inhibition chemistry in the proteolytic site combined with a direct visualization of changes in the conformational dynamics in the $26 \mathrm{~S}$ holoenzyme upon drug binding will allow for future drug design and drug screening. Still, better resolved structures of the $26 \mathrm{~S}$ proteasome are needed to identify all determinants that are involved in the long-range communication. 


\section{Appendix A}

\section{Declaration of contribution as co-author}

The inhibition mechanism of human 20S proteasomes enables next-generation inhibitor design

Schrader J, Henneberg F, Mata RA, Tittmann K, Schneider TR, Stark H, Bourenkov G, Chari A: Science, 353 (6299): 594-598

Thesis author's contributions

Conception: $20 \%$

Experimental contribution: $20 \%$

Formulation of results: $\quad 10 \%$

Long-range allosteric regulation of the human $26 \mathrm{~S}$ proteasome by $20 \mathrm{~S}$ proteasometargeting cancer drugs

Haselbach D, Schrader J, Lambrecht F, Henneberg F, Chari A, Stark H: Nat. Commun., 8: 15578

Thesis author's contributions

Conception: $\quad 20 \%$

Experimental contribution: $20 \%$

Formulation of results: $\quad 10 \%$ 


\section{Appendix B}

\section{Abbreviations}

2D two-dimensional

3D three-dimensional

Å Ångström

AAA + ATPases Associated with diverse cellular Activities

Arg Arginine

Asp Aspartic acid

ATP Adenosine triphosphate

Blm10 bleomycin-sensitive 10

$\mathrm{Br} / \mathrm{Br}^{-}$Bromine/Bromide

BS3 Bis(sulfosuccinimidyl)suberat

B. stearothermophilus Bacillus stearothermophilus

CCD Charge Coupled Device

Cdc48 Cell division cycle 48

CP Core Particle (20S proteasome)

cryo-EM cryogenic electron microscopy/ electron cryomicroscopy

CTF Contrast Transfer Function

DDi1 DNA damage-inducible 1

DNA Desoxy-Ribonucleic Acid

Dsk2 dominant suppressor of Kar2

DTT Dithiothreitol

DUB Deubiquinating enzyme

E1 Ubiquitin-activating enzyme 
E2 Ubiquitin-conjugating enzyme

E3 Ubiquitin-ligase

E3BP E3-binding protein

EM Electron Microscopy

EMDB Electron Microscopy Data Bank

FDA Food and Drug Administration

FSC Fourier shell correlation

Glu Glutamic acid

Gly Glycine

GraFix Gradient Fixation

HbYX hydrophobic, tyrosine, any amino acid tripeptide

HECT Homologous to the E6AP Carboxyl Terminus

HeLa cell line, Henrietta Lacks

Hul5 HECT ubiquitin ligase 5

IDP Intrinsically disordered proteins

I $\varkappa$ B NF-kappa-B inhibitor

Ile Isoleucine

K Lysine

kDa kiloDalton

keV kiloelectron volt

Lys Lysine

$\mathbf{I N}_{2}$ liquid nitrogen

MAD Multiple Anomalous Dispersion

Mg Magnesium

MGy Megagray

MIR Multiple Isomorphous Replacement

Mn Manganese

MPN Mpr1/Pad1 N-terminal 
N Nitrogen

$\mathrm{Nf}-\varkappa \mathrm{B}$ nuclear factor of $\varkappa \mathrm{B}$

NMA Normal Mode Analysis

NTD N-terminal domain

Ntn N-terminal nucleophile

O Oxygen

PA Proteasome Activator

PAN Proteasome-Activating Nucleotidase

PC Proteasome/Cyclosome repeats

PCI Proteasome-CSN-eIF3

PCA principal component analysis

PDB Protein Data Bank

PRU Pleckstrin-like Receptor for Ubiquitin

Rad23 Radiation Sensitive 23

RING Really Interesting New Gene

RP Regulatory Particle (19S subunit)

Rpn Regulatory particle non-ATPase

Rpt Regulatory particle tripleA-ATPase

SAD Single Anomalous Dispersion

S. cerevisiae Saccharomyces cerevisiae

SIR Single Isomorphous Replacement

SDS-PAGE Sodium Dodecyl Sulfate- Poly-Acrylamide Gel Electrophoresis

Ser Serine

SNR Signal-to-Noise-Ratio

T. acidophilum Thermoplasma acidophilum

Thr Threonine

TEM Transmission Electron Microscope

T. thermophilus Thermus thermophilus 
Ub Ubiquitin

UBA Ubiquitin-Associated

UBL Ubiquitin-Like

Ubp Ubiquitin-specific protease

UIM Ubiquitin-Interacting Motif

UPS Ubiquitin-Proteasome-System

USP Ubiquitin-Specific Protease

VCP Valosin-Containing Protein

vWA von Willebrand factor type A 


\section{Bibliography}

[1] T.-W. Mu, D. S. T. Ong, Y.-J. Wang, W. E. Balch, J. R. Yates, L. Segatori, and J. W. Kelly, "Chemical and Biological Approaches Synergize to Ameliorate ProteinFolding Diseases," Cell, vol. 134, no. 5, pp. 769-781, 2008.

[2] E. Cohen, J. F. Paulsson, P. Blinder, T. Burstyn-Cohen, D. Du, G. Estepa, A. Adame, H. M. Pham, M. Holzenberger, J. W. Kelly, E. Masliah, and A. Dillin, "Reduced IGF-1 Signaling Delays Age-Associated Proteotoxicity in Mice," Cell, vol. 139, no. 6, pp. 1157-1169, dec 2009.

[3] M. H. Glickman and A. Ciechanover, "The Ubiquitin-Proteasome Proteolytic Pathway: Destruction for the Sake of Construction," Physiological Reviews, vol. 82, no. 2, pp. 373-428, apr 2002.

[4] K. L. Rock, C. Gramm, L. Rothstein, K. Clark, R. Stein, L. Dick, D. Hwang, and A. L. Goldberg, "Inhibitors of the proteasome block the degradation of most cell proteins and the generation of peptides presented on MHC class I molecules." Cell, vol. 78, no. 5, pp. 761-71, sep 1994.

[5] D. Finley, "Recognition and processing of ubiquitin-protein conjugates by the proteasome." Annual review of biochemistry, vol. 78, pp. 477-513, jan 2009.

[6] O. Coux, K. Tanaka, and A. L. Goldberg, "Structure and Functions of the 20S and 26S Proteasomes," Annual Review of Biochemistry, vol. 65, no. 1, pp. 801-847, jun 1996.

[7] C. M. Pickart and R. E. Cohen, "Proteasomes and their kin: proteases in the machine age," Nature Reviews Molecular Cell Biology, vol. 5, no. 3, pp. 177-187, mar 2004.

[8] V. Chau, J. Tobias, A. Bachmair, D. Marriott, D. Ecker, D. Gonda, and A. Varshavsky, "A multiubiquitin chain is confined to specific lysine in a targeted shortlived protein," Science, vol. 243, no. 4898, pp. 1576-1583, mar 1989.

[9] J. S. Thrower, L. Hoffman, M. Rechsteiner, and C. M. Pickart, "Recognition of the polyubiquitin proteolytic signal." The EMBO journal, vol. 19, no. 1, pp. 94-102, jan 2000 . 
[10] A. Hershko, H. Heller, E. Eytan, and Y. Reiss, "The protein substrate binding site of the ubiquitin-protein ligase system." The Journal of biological chemistry, vol. 261, no. 26, pp. 11992-9, sep 1986.

[11] K. D. Wilkinson, "Regulation of ubiquitin-dependent processes by deubiquitinating enzymes." FASEB journal : official publication of the Federation of American Societies for Experimental Biology, vol. 11, no. 14, pp. 1245-56, dec 1997.

[12] C. M. Pickart and I. A. Rose, "Ubiquitin carboxyl-terminal hydrolase acts on ubiquitin carboxyl-terminal amides." The Journal of biological chemistry, vol. 260, no. 13 , pp. 7903-10, jul 1985.

[13] J. M. Huibregtse, M. Scheffner, S. Beaudenon, and P. M. Howley, "A family of proteins structurally and functionally related to the E6-AP ubiquitin-protein ligase." Proceedings of the National Academy of Sciences of the United States of America, vol. 92, no. 7, pp. 2563-7, mar 1995.

[14] K. L. Lorick, J. P. Jensen, S. Fang, A. M. Ong, S. Hatakeyama, and A. M. Weissman, "RING fingers mediate ubiquitin-conjugating enzyme (E2)-dependent ubiquitination." Proceedings of the National Academy of Sciences of the United States of America, vol. 96, no. 20, pp. 11364-9, sep 1999.

[15] T. Ravid and M. Hochstrasser, "Diversity of degradation signals in the ubiquitinproteasome system," Nature Reviews Molecular Cell Biology, vol. 9, no. 9, pp. 679689 , sep 2008.

[16] M. Groll, L. Ditzel, J. Löwe, D. Stock, M. Bochtler, H. D. Bartunik, and R. Huber, "Structure of 20S proteasome from yeast at 2.4 A resolution." Nature, vol. 386, no. 6624, pp. 463-71, apr 1997.

[17] M. Groll, M. H. Glickman, D. Finley, M. Bajorek, A. Köhler, L. Moroder, D. M. Rubin, and R. Huber, "A gated channel into the proteasome core particle," Nature Structural Biology, vol. 7, no. 11, pp. 1062-1067, nov 2000.

[18] F. Kriegenburg, E. G. Poulsen, A. Koch, E. Krüger, and R. Hartmann-Petersen, "Redox Control of the Ubiquitin-Proteasome System: From Molecular Mechanisms to Functional Significance," Antioxidants \& Redox Signaling, vol. 15, no. 8, pp. 2265-2299, oct 2011.

[19] M. Orlowski and C. Michaud, "Pituitary multicatalytic proteinase complex. Specificity of components and aspects of proteolytic activity," Biochemistry, vol. 28, no. 24, pp. 9270-9278, nov 1989.

[20] A. K. Nussbaum, T. P. Dick, W. Keilholz, M. Schirle, S. Stevanović, K. Dietz, W. Heinemeyer, M. Groll, D. H. Wolf, R. Huber, H. G. Rammensee, and H. Schild, 
"Cleavage motifs of the yeast 20S proteasome beta subunits deduced from digests of enolase 1." Proceedings of the National Academy of Sciences of the United States of America, vol. 95, no. 21, pp. 12 504-9, oct 1998.

[21] I. Schechter and A. Berger, "On the size of the active site in proteases. I. Papain," Biochemical and Biophysical Research Communications, vol. 27, no. 2, pp. 157-162, apr 1967.

[22] E. M. Huber, M. Basler, R. Schwab, W. Heinemeyer, C. J. Kirk, M. Groettrup, and M. Groll, "Immuno- and constitutive proteasome crystal structures reveal differences in substrate and inhibitor specificity." Cell, vol. 148, no. 4, pp. 727-38, feb 2012.

[23] M. Unno, T. Mizushima, Y. Morimoto, Y. Tomisugi, K. Tanaka, N. Yasuoka, and T. Tsukihara, "The Structure of the Mammalian 20S Proteasome at $2.75 \AA$ Resolution," Structure, vol. 10, no. 5, pp. 609-618, may 2002.

[24] J. Löwe, D. Stock, B. Jap, P. Zwickl, W. Baumeister, and R. Huber, "Crystal structure of the $20 \mathrm{~S}$ proteasome from the archaeon T. acidophilum at 3.4 A resolution." Science, vol. 268, no. 5210, pp. 533-9, apr 1995.

[25] W. Harshbarger, C. Miller, C. Diedrich, and J. Sacchettini, "Crystal structure of the human 20S proteasome in complex with carfilzomib." Structure, vol. 23, no. 2, pp. 418-24, feb 2015.

[26] E. Seemüller, A. Lupas, D. Stock, J. Löwe, R. Huber, and W. Baumeister, "Proteasome from Thermoplasma acidophilum: a threonine protease." Science, vol. 268, no. 5210, pp. 579-82, apr 1995.

[27] A. Martin, T. A. Baker, and R. T. Sauer, "Rebuilt AAA + motors reveal operating principles for ATP-fuelled machines." Nature, vol. 437, no. 7062, pp. 1115-20, oct 2005.

[28] R. J. Tomko, M. Funakoshi, K. Schneider, J. Wang, M. Hochstrasser, and M. Hochstrasser, "Heterohexameric ring arrangement of the eukaryotic proteasomal ATPases: implications for proteasome structure and assembly." Molecular cell, vol. 38, no. 3, pp. 393-403, may 2010.

[29] K. Hofmann and P. Bucher, "The PCI domain: a common theme in three multiprotein complexes," Trends in Biochemical Sciences, vol. 23, no. 6, pp. 204-205, jun 1998.

[30] K. Paraskevopoulos, F. Kriegenburg, M. H. Tatham, H. I. Rösner, B. Medina, I. B. Larsen, R. Brandstrup, K. G. Hardwick, R. T. Hay, B. B. Kragelund, R. Hartmann- 
Petersen, and C. Gordon, "Dss1 is a 26S proteasome ubiquitin receptor." Molecular cell, vol. 56, no. 3, pp. 453-61, nov 2014.

[31] G. C. Lander, E. Estrin, M. E. Matyskiela, C. Bashore, E. Nogales, and A. Martin, "Complete subunit architecture of the proteasome regulatory particle," Nature, vol. 482 , jan 2012.

[32] R. Verma, L. Aravind, R. Oania, W. H. McDonald, J. R. Yates, E. V. Koonin, and R. J. Deshaies, "Role of Rpn11 metalloprotease in deubiquitination and degradation by the $26 \mathrm{~S}$ proteasome." Science, vol. 298, no. 5593, pp. 611-5, oct 2002.

[33] M. Schmidt, J. Hanna, S. Elsasser, and D. Finley, "Proteasome-associated proteins: regulation of a proteolytic machine," Biological Chemistry, vol. 386, no. 8, pp. 725737 , jan 2005.

[34] R. Verma, R. Oania, J. Graumann, and R. J. Deshaies, "Multiubiquitin Chain Receptors Define a Layer of Substrate Selectivity in the Ubiquitin-Proteasome System," Cell, vol. 118, no. 1, pp. 99-110, jul 2004.

[35] D. S. Leggett, J. Hanna, A. Borodovsky, B. Crosas, M. Schmidt, R. T. Baker, T. Walz, H. Ploegh, and D. Finley, "Multiple Associated Proteins Regulate Proteasome Structure and Function," Molecular Cell, vol. 10, no. 3, pp. 495-507, sep 2002.

[36] J. You and C. M. Pickart, "A HECT domain E3 enzyme assembles novel polyubiquitin chains." The Journal of biological chemistry, vol. 276, no. 23, pp. 19871-8, jun 2001.

[37] D. M. Smith, S.-C. Chang, S. Park, D. Finley, Y. Cheng, and A. L. Goldberg, "Docking of the proteasomal ATPases' carboxyl termini in the 20S proteasome's alpha ring opens the gate for substrate entry." Molecular cell, vol. 27, no. 5, pp. 731-44, sep 2007.

[38] G. Tian, S. Park, M. J. Lee, B. Huck, F. McAllister, C. P. Hill, S. P. Gygi, and D. Finley, "An asymmetric interface between the regulatory and core particles of the proteasome." Nature structural \& molecular biology, vol. 18, no. 11, pp. 1259-67, oct 2011.

[39] B. M. Stadtmueller and C. P. Hill, "Proteasome activators." Molecular cell, vol. 41, no. 1, pp. 8-19, jan 2011.

[40] D. Barthelme and R. T. Sauer, "Identification of the Cdc48/20S proteasome as an ancient AAA+ proteolytic machine." Science, vol. 337, no. 6096, pp. 843-6, aug 2012. 
[41] D. Barthelme and R. T. Sauer, "Bipartite determinants mediate an evolutionarily conserved interaction between Cdc48 and the 20S peptidase." Proceedings of the National Academy of Sciences of the United States of America, vol. 110, no. 9, pp. 3327-32, feb 2013.

[42] S. Bohn, F. Beck, E. Sakata, T. Walzthoeni, M. Beck, R. Aebersold, F. Förster, W. Baumeister, and S. Nickell, "Structure of the 26S proteasome from Schizosaccharomyces pombe at subnanometer resolution." Proceedings of the National Academy of Sciences of the United States of America, vol. 107, no. 49, pp. 20 992-7, dec 2010.

[43] K. Lasker, F. Förster, S. Bohn, T. Walzthoeni, E. Villa, P. Unverdorben, F. Beck, R. Aebersold, A. Sali, and W. Baumeister, "Molecular architecture of the 26S proteasome holocomplex determined by an integrative approach." Proceedings of the National Academy of Sciences of the United States of America, vol. 109, no. 5, pp. 1380-7, jan 2012.

[44] P. C. A. da Fonseca, J. He, and E. P. Morris, "Molecular model of the human 26S proteasome." Molecular cell, vol. 46, no. 1, pp. 54-66, apr 2012.

[45] F. Beck, P. Unverdorben, S. Bohn, A. Schweitzer, G. Pfeifer, E. Sakata, S. Nickell, J. M. Plitzko, E. Villa, W. Baumeister, and F. Förster, "Near-atomic resolution structural model of the yeast $26 \mathrm{~S}$ proteasome." Proceedings of the National Academy of Sciences of the United States of America, vol. 109, no. 37, pp. 14870-5, sep 2012.

[46] A. Schweitzer, A. Aufderheide, T. Rudack, F. Beck, G. Pfeifer, J. M. Plitzko, E. Sakata, K. Schulten, F. Förster, and W. Baumeister, "Structure of the human 26S proteasome at a resolution of $3.9 \AA$ A." Proceedings of the National Academy of Sciences of the United States of America, vol. 113, no. 28, pp. 7816-21, jul 2016.

[47] X. Huang, B. Luan, J. Wu, and Y. Shi, "An atomic structure of the human 26S proteasome," Nature Structural \& Molecular Biology, vol. 23, no. 9, pp. 778-785, jul 2016.

[48] S. Chen, J. Wu, Y. Lu, Y.-B. Ma, B.-H. Lee, Z. Yu, Q. Ouyang, D. J. Finley, M. W. Kirschner, and Y. Mao, "Structural basis for dynamic regulation of the human 26S proteasome." Proceedings of the National Academy of Sciences of the United States of America, vol. 113, no. 46, pp. 12991-12996, nov 2016.

[49] S. Nickell, F. Beck, S. H. W. Scheres, A. Korinek, F. Förster, K. Lasker, O. Mihalache, N. Sun, I. Nagy, A. Sali, J. M. Plitzko, J.-M. Carazo, M. Mann, and W. Baumeister, "Insights into the molecular architecture of the $26 \mathrm{~S}$ proteasome." Proceedings of the National Academy of Sciences of the United States of America, vol. 106, no. 29, pp. 11943-7, jul 2009. 
[50] P. Unverdorben, F. Beck, P. Śledź, A. Schweitzer, G. Pfeifer, J. M. Plitzko, W. Baumeister, and F. Förster, "Deep classification of a large cryo-EM dataset defines the conformational landscape of the 26S proteasome." Proceedings of the National Academy of Sciences of the United States of America, vol. 111, no. 15, pp. 5544-9, apr 2014.

[51] M. E. Matyskiela, G. C. Lander, and A. Martin, "Conformational switching of the $26 \mathrm{~S}$ proteasome enables substrate degradation." Nature structural $\& 5$ molecular biology, vol. 20, no. 7, pp. 781-8, jul 2013.

[52] P. F. Bross, "Approval Summary for Bortezomib for Injection in the Treatment of Multiple Myeloma," Clinical Cancer Research, vol. 10, no. 12, pp. 3954-3964, jun 2004.

[53] International Myeloma Foundation, "Myeloma.org," 2017. [Online]. Available: https://www.myeloma.org/

[54] National Library of Medicine, "Clinicaltrials.gov," 2017. [Online]. Available: https://clinicaltrials.gov/

[55] S. T. Nawrocki, J. S. Carew, M. S. Pino, R. A. Highshaw, K. Dunner, P. Huang, J. L. Abbruzzese, and D. J. McConkey, "Bortezomib sensitizes pancreatic cancer cells to endoplasmic reticulum stress-mediated apoptosis." Cancer research, vol. 65, no. 24, pp. $11658-66$, dec 2005.

[56] B. An, R. H. Goldfarb, R. Siman, and Q. P. Dou, "Novel dipeptidyl proteasome inhibitors overcome Bcl-2 protective function and selectively accumulate the cyclindependent kinase inhibitor p27 and induce apoptosis in transformed, but not normal, human fibroblasts," Cell Death and Differentiation, vol. 5, no. 12, pp. 10621075, dec 1998.

[57] J. Adams, "Proteasome inhibition: a novel approach to cancer therapy," Trends in Molecular Medicine, vol. 8, no. 4, pp. S49-S54, apr 2002.

[58] H. C. Drexler, "Activation of the cell death program by inhibition of proteasome function." Proceedings of the National Academy of Sciences of the United States of America, vol. 94, no. 3, pp. 855-60, feb 1997.

[59] P. Masdehors, S. Omura, H. Merle-Beral, F. Mentz, J.-M. Cosset, J. Dumont, H. Magdelenat, and J. Delic, "Increased sensitivity of CLL-derived lymphocytes to apoptotic death activation by the proteasome-specific inhibitor lactacystin," British Journal of Haematology, vol. 105, no. 3, pp. 752-757, jun 1999. 
[60] P. Masdehors, H. Merle-Béral, K. Maloum, S. Omura, H. Magdelénat, and J. Delic, "Deregulation of the ubiquitin system and p53 proteolysis modify the apoptotic response in B-CLL lymphocytes." Blood, vol. 96, no. 1, pp. 269-74, jul 2000.

[61] A. A. Argyriou, G. Iconomou, and H. P. Kalofonos, "Bortezomib-induced peripheral neuropathy in multiple myeloma: a comprehensive review of the literature," Blood, vol. 112, no. 5, pp. 1593-1599, sep 2008.

[62] P. G. Richardson, B. Barlogie, J. Berenson, S. Singhal, S. Jagannath, D. Irwin, S. V. Rajkumar, G. Srkalovic, M. Alsina, R. Alexanian, D. Siegel, R. Z. Orlowski, D. Kuter, S. A. Limentani, S. Lee, T. Hideshima, D.-L. Esseltine, M. Kauffman, J. Adams, D. P. Schenkein, and K. C. Anderson, "A Phase 2 Study of Bortezomib in Relapsed, Refractory Myeloma," New England Journal of Medicine, vol. 348, no. 26, pp. 2609-2617, jun 2003.

[63] P. C. A. da Fonseca and E. P. Morris, "Cryo-EM reveals the conformation of a substrate analogue in the human 20S proteasome core." Nature communications, vol. 6, p. 7573, jan 2015 .

[64] J. Adams, M. Behnke, S. Chen, A. A. Cruickshank, L. R. Dick, L. Grenier, J. M. Klunder, Y.-T. Ma, L. Plamondon, and R. L. Stein, "Potent and selective inhibitors of the proteasome: Dipeptidyl boronic acids," Bioorganic \& Medicinal Chemistry Letters, vol. 8, no. 4, pp. 333-338, feb 1998.

[65] D. Chauhan, Z. Tian, B. Zhou, D. Kuhn, R. Orlowski, N. Raje, P. Richardson, and K. C. Anderson, "In vitro and in vivo selective antitumor activity of a novel orally bioavailable proteasome inhibitor MLN9708 against multiple myeloma cells." Clinical cancer research : an official journal of the American Association for Cancer Research, vol. 17, no. 16, pp. 5311-21, aug 2011.

[66] E. Kupperman, E. C. Lee, Y. Cao, B. Bannerman, M. Fitzgerald, A. Berger, J. Yu, Y. Yang, P. Hales, F. Bruzzese, J. Liu, J. Blank, K. Garcia, C. Tsu, L. Dick, P. Fleming, L. Yu, M. Manfredi, M. Rolfe, and J. Bolen, "Evaluation of the Proteasome Inhibitor MLN9708 in Preclinical Models of Human Cancer," Cancer Research, vol. 70, no. 5, pp. 1970-1980, mar 2010.

[67] R. Piva, B. Ruggeri, M. Williams, G. Costa, I. Tamagno, D. Ferrero, V. Giai, M. Coscia, S. Peola, M. Massaia, G. Pezzoni, C. Allievi, N. Pescalli, M. Cassin, S. di Giovine, P. Nicoli, P. de Feudis, I. Strepponi, I. Roato, R. Ferracini, B. Bussolati, G. Camussi, S. Jones-Bolin, K. Hunter, H. Zhao, A. Neri, A. Palumbo, C. Berkers, H. Ovaa, A. Bernareggi, and G. Inghirami, "CEP-18770: A novel, orally active proteasome inhibitor with a tumor-selective pharmacologic profile competitive with bortezomib," Blood, vol. 111, no. 5, pp. 2765-2775, mar 2008. 
[68] J. Adams, "The development of proteasome inhibitors as anticancer drugs." Cancer cell, vol. 5, no. 5, pp. 417-21, may 2004.

[69] M. Altun, P. J. Galardy, R. Shringarpure, T. Hideshima, R. LeBlanc, K. C. Anderson, H. L. Ploegh, and B. M. Kessler, "Effects of PS-341 on the activity and composition of proteasomes in multiple myeloma cells." Cancer research, vol. 65, no. 17, pp. 7896-901, sep 2005.

[70] M. Groll, K. B. Kim, N. Kairies, R. Huber, and C. M. Crews, "Crystal Structure of Epoxomicin:20S Proteasome Reveals a Molecular Basis for Selectivity of $\alpha^{\prime}, \beta^{\prime}-$ Epoxyketone Proteasome Inhibitors," Journal of the American Chemical Society, vol. 122 , no. 6 , pp. $1237-1238$, feb 2000.

[71] S. D. Demo, C. J. Kirk, M. A. Aujay, T. J. Buchholz, M. Dajee, M. N. Ho, J. Jiang, G. J. Laidig, E. R. Lewis, F. Parlati, K. D. Shenk, M. S. Smyth, C. M. Sun, M. K. Vallone, T. M. Woo, C. J. Molineaux, and M. K. Bennett, "Antitumor activity of PR-171, a novel irreversible inhibitor of the proteasome." Cancer research, vol. 67, no. 13, pp. 6383-91, jul 2007.

[72] M. Verdoes, L. I. Willems, W. A. van der Linden, B. A. Duivenvoorden, G. A. van der Marel, B. I. Florea, A. F. Kisselev, and H. S. Overkleeft, "A panel of subunit-selective activity-based proteasome probes." Organic $\&$ biomolecular chemistry, vol. 8, no. 12, pp. 2719-27, jun 2010.

[73] M. A. Gräwert, N. Gallastegui, M. Stein, B. Schmidt, P.-M. Kloetzel, R. Huber, and M. Groll, "Elucidation of the $\alpha$-keto-aldehyde binding mechanism: a lead structure motif for proteasome inhibition." Angewandte Chemie (International ed. in English), vol. 50, no. 2, pp. 542-4, jan 2011.

[74] S. Imajohohmi, T. Kawaguchi, S. Sugiyama, K. Tanaka, S. Omura, and H. Kikuchi, "Lactacystin, a Specific Inhibitor of the Proteasome, Induces Apoptosis in Human Monoblast U937 Cells," Biochemical and Biophysical Research Communications, vol. 217, no. 3, pp. 1070-1077, dec 1995.

[75] K. Shinohara, M. Tomioka, H. Nakano, S. Toné, H. Ito, and S. Kawashima, "Apoptosis induction resulting from proteasome inhibition." The Biochemical journal, pp. 385-8, jul 1996.

[76] C. GarcÄśìįa-EcheverrÄśìĄa, P. Imbach, D. France, P. Fürst, M. Lang, M. Noorani, D. Scholz, J. Zimmermann, and P. Furet, "A New Structural Class of Selective and Non-covalent Inhibitors of the Chymotrypsin-like Activity of the 20S Proteasome," Bioorganic \& Medicinal Chemistry Letters, vol. 11, no. 10, pp. 1317-1319, may 2001. 
[77] P. Furet, P. Imbach, M. Noorani, J. Koeppler, K. Laumen, M. Lang, V. Guagnano, P. Fuerst, J. Roesel, J. Zimmermann, and C. García-Echeverría, "Entry into a New Class of Potent Proteasome Inhibitors Having High Antiproliferative Activity by Structure-Based Design," Journal of Medicinal Chemistry, vol. 47, no. 20, pp. 4810-4813, sep 2004.

[78] B. Villoutreix, M. Reboud-Ravaux, N. Basse, J. Vidal, and M. Montes, "Nitrogen heterocycle derivatives as proteasome modulators," 2009.

[79] H. R. Lawrence, S. M. Sebti, and S. Ozcan, "Proteasome chymotrypsin-like inhibition using pi-1833 analogs," 2012.

[80] Y. Koguchi, J. Kohno, M. Nishio, K. Takahashi, T. Okuda, T. Ohnuki, and S. Komatsubara, "TMC-95A, B, C, and D, Novel Proteasome Inhibitors Produced by Apiospora montagnei Sacc. TC 1093. Taxonomy, Production, Isolation, and Biological Activities." The Journal of Antibiotics, vol. 53, no. 2, pp. 105-109, 2000.

[81] S. Schoof, G. Pradel, M. Aminake, B. Ellinger, S. Baumann, M. Potowski, Y. Najajreh, M. Kirschner, and H.-D. Arndt, "Antiplasmodial Thiostrepton Derivatives: Proteasome Inhibitors with a Dual Mode of Action," Angewandte Chemie International Edition, vol. 49, no. 19, pp. 3317-3321, apr 2010.

[82] C. Blackburn, K. M. Gigstad, P. Hales, K. Garcia, M. Jones, F. J. Bruzzese, C. Barrett, J. X. Liu, T. A. Soucy, D. S. Sappal, N. Bump, E. J. Olhava, P. Fleming, L. R. Dick, C. Tsu, M. D. Sintchak, and J. L. Blank, "Characterization of a new series of non-covalent proteasome inhibitors with exquisite potency and selectivity for the 20S B5-subunit," Biochemical Journal, vol. 430, no. 3, pp. 461-476, sep 2010.

[83] J. Kohno, Y. Koguchi, M. Nishio, K. Nakao, M. Kuroda, R. Shimizu, T. Ohnuki, and S. Komatsubara, "Structures of TMC-95A-D: Novel Proteasome Inhibitors from Apiospora montagnei Sacc. TC 1093," The Journal of Organic Chemistry, vol. 65, 2000.

[84] M. Groll, Y. Koguchi, R. Huber, and J. Kohno, "Crystal structure of the $20 \mathrm{~S}$ proteasome:TMC-95A complex: a non-covalent proteasome inhibitor," Journal of Molecular Biology, vol. 311, no. 3, pp. 543-548, aug 2001.

[85] B. E. Clurman, R. J. Sheaff, K. Thress, M. Groudine, and J. M. Roberts, "Turnover of cyclin E by the ubiquitin-proteasome pathway is regulated by cdk2 binding and cyclin phosphorylation." Genes $\&$ development, vol. 10, no. 16, pp. 1979-90, aug 1996.

[86] M. Glotzer, A. W. Murray, and M. W. Kirschner, "Cyclin is degraded by the ubiquitin pathway," Nature, vol. 349, no. 6305, pp. 132-138, jan 1991. 
[87] C. Li, R. Dai, and D. Longo, "Inactivation of NF- $\kappa \mathrm{B}$ Inhibitor $\mathrm{I} \kappa \mathrm{B} \alpha$ : UbiquitinDependent Proteolysis and Its Degradation Product," Biochemical and Biophysical Research Communications, vol. 215, no. 1, pp. 292-301, oct 1995.

[88] V. J. Palombella, O. J. Rando, A. L. Goldberg, and T. Maniatis, "The ubiquitinproteasome pathway is required for processing the NF- $\kappa \mathrm{B} 1$ precursor protein and the activation of NF- $\kappa$ B," Cell, vol. 78, no. 5, pp. 773-785, sep 1994.

[89] D. R. Chowdary, J. J. Dermody, K. K. Jha, and H. L. Ozer, "Accumulation of p53 in a mutant cell line defective in the ubiquitin pathway." Molecular and cellular biology, vol. 14, no. 3, pp. 1997-2003, mar 1994.

[90] M. Ljungman, "Dial 9-1-1 for p53: mechanisms of p53 activation by cellular stress." Neoplasia, vol. 2, no. 3, pp. 208-25, 2000.

[91] J. C. Phillips, A. Wlodawer, M. M. Yevitz, and K. O. Hodgson, "Applications of synchrotron radiation to protein crystallography: preliminary results." Proceedings of the National Academy of Sciences of the United States of America, vol. 73, no. 1, pp. 128-32, jan 1976.

[92] J. C. Kendrew, G. Bodo, H. M. Dintzis, R. G. Parrish, H. Wyckoff, and D. C. Phillips, "A Three-Dimensional Model of the Myoglobin Molecule Obtained by XRay Analysis," Nature, vol. 181, no. 4610, pp. 662-666, mar 1958.

[93] M. F. Perutz, M. G. Rossmann, A. F. Cullis, H. Muirhead, G. Will, and A. C. T. North, "Structure of Hæmoglobin: A Three-Dimensional Fourier Synthesis at 5.5-A. Resolution, Obtained by X-Ray Analysis," Nature, vol. 185, no. 4711, pp. 416-422, feb 1960 .

[94] L. Bragg and M. F. Perutz, "The Structure of Haemoglobin." Proceedings of the Royal Society of London A: Mathematical, Physical and Engineering Sciences, vol. 225, no. 1162, 1954.

[95] D. W. Green, V. M. Ingram, and M. F. Perutz, "The Structure of Haemoglobin." Proceedings of the Royal Society of London A: Mathematical, Physical and Engineering Sciences, vol. 225, no. 1162, 1954.

[96] G. Taylor, "The phase problem," Acta Crystallographica Section D Biological Crystallography, vol. 59, no. 11, pp. 1881-1890, nov 2003.

[97] G. Scapin, "Molecular replacement then and now." Acta crystallographica. Section D, Biological crystallography, vol. 69, no. Pt 11, pp. 2266-75, nov 2013. 
[98] J. C. Kendrew, G. Bodo, H. M. Dintzis, R. G. Parrish, H. Wyckoff, and D. C. Phillips, "A Three-Dimensional Model of the Myoglobin Molecule Obtained by XRay Analysis," Nature, vol. 181, no. 4610, pp. 662-666, mar 1958.

[99] W. A. Hendrickson and M. M. Teeter, "Structure of the hydrophobic protein crambin determined directly from the anomalous scattering of sulphur," Nature, vol. 290, no. 5802, pp. 107-113, mar 1981.

[100] W. Hendrickson, "Determination of macromolecular structures from anomalous diffraction of synchrotron radiation," Science, vol. 254, no. 5028, pp. 51-58, oct 1991.

[101] A. Yonath, J. Missing, B. Tesche, S. Lorenz, V. Erdmann, and H. Wittmann, "Crystallization of the large ribosomal subunit from Bacillus Stearothermophilus," Biochemistry International, vol. 1, no. 5, pp. 428-435, 1980.

[102] J. H. Cate, "X-ray Crystal Structures of 70S Ribosome Functional Complexes," Science, vol. 285, no. 5436, pp. 2095-2104, sep 1999.

[103] J. Bernal and D. Crowfoot, "X-Ray Photographs of Crystalline Pepsin," Nature, vol. 133, pp. 794-795, 1934.

[104] B. Heras and J. L. Martin, "Post-crystallization treatments for improving diffraction quality of protein crystals," Acta Crystallographica Section D Biological Crystallography, vol. 61, no. 9, pp. 1173-1180, sep 2005.

[105] M. Knoll and E. Ruska, "Das Elektronenmikroskop," Zeitschrift für Physik, vol. 78, no. 5-6, pp. 318-339, may 1932.

[106] J. Dubochet, J. Lepault, R. Freeman, J. A. Berriman, and J.-C. Homo, "Electron microscopy of frozen water and aqueous solutions," Journal of Microscopy, vol. 128, no. 3, pp. 219-237, dec 1982.

[107] H. Stark, F. Zemlin, and C. Boettcher, "Electron radiation damage to protein crystals of bacteriorhodopsin at different temperatures," Ultramicroscopy, vol. 63, no. 2, pp. 75-79, 1996.

[108] W. Wong, X.-c. Bai, A. Brown, I. S. Fernandez, E. Hanssen, M. Condron, Y. H. Tan, J. Baum, and S. H. Scheres, "Cryo-EM structure of the Plasmodium falciparum 80S ribosome bound to the anti-protozoan drug emetine," eLife, vol. 3, p. e01963, jun 2014 .

[109] P. Lu, X.-c. Bai, D. Ma, T. Xie, C. Yan, L. Sun, G. Yang, Y. Zhao, R. Zhou, S. H. W. Scheres, and Y. Shi, "Three-dimensional structure of human $\gamma$-secretase," Nature, vol. 512, no. 7513, pp. 166-170, jun 2014. 
[110] D. Stalling, M. Westerhoff, and H. C. Hege, "Amira: a highly interactive system for visual data analysis," 2005.

[111] P. Emsley and K. Cowtan, "Coot: model-building tools for molecular graphics." Acta crystallographica. Section D, Biological crystallography, vol. 60, pp. 2126-32, $\operatorname{dec} 2004$.

[112] K. Zhang, "Gctf: Real-time CTF determination and correction," Journal of Structural Biology, vol. 193, no. 1, pp. 1-12, jan 2016.

[113] M. van Heel, G. Harauz, E. V. Orlova, R. Schmidt, and M. Schatz, "A New Generation of the IMAGIC Image Processing System," Journal of Structural Biology, vol. 116, no. 1, pp. 17-24, jan 1996.

[114] A. A. Lebedev, P. Young, M. N. Isupov, O. V. Moroz, A. A. Vagin, and G. N. Murshudov, "JLigand: a graphical tool for the CCP4 template-restraint library." Acta crystallographica. Section D, Biological crystallography, vol. 68, no. Pt 4, pp. 431-40, apr 2012.

[115] S. H. W. Scheres, "RELION: implementation of a Bayesian approach to cryo-EM structure determination." Journal of structural biology, vol. 180, no. 3, pp. 519-30, $\operatorname{dec} 2012$.

[116] H. Elmlund, D. Elmlund, and S. Bengio, "PRIME: probabilistic initial 3D model generation for single-particle cryo-electron microscopy." Structure, vol. 21, no. 8, pp. 1299-306, aug 2013.

[117] E. F. Pettersen, T. D. Goddard, C. C. Huang, G. S. Couch, D. M. Greenblatt, E. C. Meng, and T. E. Ferrin, "UCSF Chimera-a visualization system for exploratory research and analysis." Journal of computational chemistry, vol. 25, no. 13, pp. $1605-12$, oct 2004.

[118] J. D. Dignam, R. M. Lebovitz, and R. G. Roeder, "Accurate transcription initiation by RNA polymerase II in a soluble extract from isolated mammalian nuclei." Nucleic acids research, vol. 11, no. 5, pp. 1475-89, mar 1983.

[119] U. K. Laemmli, "Cleavage of structural proteins during the assembly of the head of bacteriophage T4." Nature, vol. 227, no. 5259, pp. 680-5, aug 1970.

[120] J. Schrader, F. Henneberg, R. A. Mata, K. Tittmann, T. R. Schneider, H. Stark, G. Bourenkov, and A. Chari, "The inhibition mechanism of human 20S proteasomes enables next-generation inhibitor design," Science, vol. 353, no. 6299, pp. 594-598, aug 2016. 
[121] F. Weigend and R. Ahlrichs, "Balanced basis sets of split valence, triple zeta valence and quadruple zeta valence quality for H to Rn: Design and assessment of accuracy," Physical Chemistry Chemical Physics, vol. 7, no. 18, p. 3297, 2005.

[122] C. Lee, W. Yang, and R. G. Parr, "Development of the Colle-Salvetti correlationenergy formula into a functional of the electron density," Physical Review B, vol. 37, no. 2, pp. 785-789, jan 1988.

[123] A. D. Becke, "Densityâ̆̆̌rfunctional thermochemistry. III. The role of exact exchange," The Journal of Chemical Physics, vol. 98, no. 7, pp. 5648-5652, apr 1993.

[124] S. Grimme, S. Ehrlich, and L. Goerigk, "Effect of the damping function in dispersion corrected density functional theory," Journal of Computational Chemistry, vol. 32, no. 7 , pp. 1456-1465, may 2011.

[125] A. D. Becke and E. R. Johnson, "A simple effective potential for exchange," The Journal of Chemical Physics, vol. 124, no. 22, p. 221101, jun 2006.

[126] A. Klamt and G. Schüürmann, "COSMO: a new approach to dielectric screening in solvents with explicit expressions for the screening energy and its gradient," $J$. Chem. Soc., Perkin Trans. 2, no. 5, pp. 799-805, 1993.

[127] M. R. A. Blomberg, T. Borowski, F. Himo, R.-Z. Liao, and P. E. M. Siegbahn, "Quantum Chemical Studies of Mechanisms for Metalloenzymes," Chemical Reviews, vol. 114, no. 7, pp. 3601-3658, apr 2014.

[128] F. Neese, "The ORCA program system," Wiley Interdisciplinary Reviews: Computational Molecular Science, vol. 2, no. 1, pp. 73-78, jan 2012.

[129] B. Rupp, Biomolecular crystallography : principles, practice, and application to structural biology. New York: Garland Science, 2010.

[130] A. A. Vagin, R. A. Steiner, A. A. Lebedev, L. Potterton, S. McNicholas, F. Long, and G. N. Murshudov, "REFMAC 5 dictionary: organization of prior chemical knowledge and guidelines for its use," Acta Crystallographica Section D Biological Crystallography, vol. 60, no. 12, pp. 2184-2195, dec 2004.

[131] T. Grant and N. Grigorieff, "Measuring the optimal exposure for single particle cryo-EM using a 2.6 A reconstruction of rotavirus VP6." eLife, vol. 4, p. e06980, jan 2015.

[132] K. Zhang, "Gautomatch." [Online]. Available: http://www.mrc-lmb.cam.ac.uk/ kzhang/

[133] A. Kucukelbir, F. J. Sigworth, and H. D. Tagare, "Quantifying the local resolution of cryo-EM density maps." Nature methods, vol. 11, no. 1, pp. 63-5, jan 2014. 
[134] Y. Song, F. DiMaio, R. Y.-R. Wang, D. Kim, C. Miles, T. Brunette, J. Thompson, and D. Baker, "High-resolution comparative modeling with RosettaCM." Structure, vol. 21 , no. 10 , pp. $1735-42$, oct 2013.

[135] D. E. Kim, D. Chivian, and D. Baker, "Protein structure prediction and analysis using the Robetta server." Nucleic acids research, vol. 32, pp. W526-31, jul 2004.

[136] F. Zhang, M. Hu, G. Tian, P. Zhang, D. Finley, P. D. Jeffrey, and Y. Shi, "Structural insights into the regulatory particle of the proteasome from Methanocaldococcus jannaschii." Molecular cell, vol. 34, no. 4, pp. 473-84, may 2009.

[137] L. J. McGuffin, K. Bryson, and D. T. Jones, "The PSIPRED protein structure prediction server." Bioinformatics (Oxford, England), vol. 16, no. 4, pp. 404-5, apr 2000.

[138] P. D. Adams, P. V. Afonine, G. Bunkóczi, V. B. Chen, I. W. Davis, N. Echols, J. J. Headd, L.-W. Hung, G. J. Kapral, R. W. Grosse-Kunstleve, A. J. McCoy, N. W. Moriarty, R. Oeffner, R. J. Read, D. C. Richardson, J. S. Richardson, T. C. Terwilliger, and P. H. Zwart, "PHENIX: a comprehensive Python-based system for macromolecular structure solution." Acta crystallographica. Section D, Biological crystallography, vol. 66, no. Pt 2, pp. 213-21, feb 2010.

[139] S. H. W. Scheres, "Chapter Six - Processing of Structurally Heterogeneous Cryo-EM Data in RELION," in Methods in Enzymology, 2016, vol. 579, pp. 125-157.

[140] P. A. Penczek, M. Kimmel, and C. M. T. Spahn, "Identifying conformational states of macromolecules by eigen-analysis of resampled cryo-EM images." Structure, vol. 19, no. 11, pp. 1582-90, nov 2011.

[141] P. I. Zhuravlev and G. A. Papoian, "Protein functional landscapes, dynamics, allostery: a tortuous path towards a universal theoretical framework," Quarterly Reviews of Biophysics, vol. 43, no. 03, pp. 295-332, aug 2010.

[142] D. L. Beveridge and F. M. DiCapua, "Free Energy Via Molecular Simulation: Applications to Chemical and Biomolecular Systems," Annual Review of Biophysics and Biophysical Chemistry, vol. 18, no. 1, pp. 431-492, jun 1989.

[143] E. M. Huber, M. Basler, R. Schwab, W. Heinemeyer, C. J. Kirk, M. Groettrup, and M. Groll, "Immuno- and Constitutive Proteasome Crystal Structures Reveal Differences in Substrate and Inhibitor Specificity," Cell, vol. 148, no. 4, pp. 727-738, feb 2012.

[144] R. Z. Orlowski and D. J. Kuhn, "Proteasome Inhibitors in Cancer Therapy: Lessons from the First Decade," Clinical Cancer Research, vol. 14, no. 6, pp. 1649-1657, mar 2008. 
[145] M. F. Kleijnen, J. Roelofs, S. Park, N. A. Hathaway, M. Glickman, R. W. King, and D. Finley, "Stability of the proteasome can be regulated allosterically through engagement of its proteolytic active sites." Nature structural \& molecular biology, vol. 14 , no. 12 , pp. $1180-8$, dec 2007.

[146] D. Haselbach, J. Schrader, F. Lambrecht, F. Henneberg, A. Chari, and H. Stark, "Long-range allosteric regulation of the human $26 \mathrm{~S}$ proteasome by $20 \mathrm{~S}$ proteasometargeting cancer drugs," Nature Communications, vol. 8, p. 15578, may 2017.

[147] C. Haglund, C. Mohanty, M. Fryknäs, P. D’Arcy, R. Larsson, S. Linder, and L. Rickardson, "Identification of an inhibitor of the ubiquitin-proteasome system that induces accumulation of polyubiquitinated proteins in the absence of blocking of proteasome function," MedChem Comm, vol. 5, no. 3, p. 376, 2014.

[148] A. Chari, D. Haselbach, J.-M. Kirves, J. Ohmer, E. Paknia, N. Fischer, O. Ganichkin, V. Möller, J. J. Frye, G. Petzold, M. Jarvis, M. Tietzel, C. Grimm, J.-M. Peters, B. A. Schulman, K. Tittmann, J. Markl, U. Fischer, and H. Stark, "ProteoPlex: stability optimization of macromolecular complexes by sparse-matrix screening of chemical space." Nature methods, vol. 12, no. 9, pp. 859-65, sep 2015.

[149] J. R. Casey, S. Grinstein, and J. Orlowski, "Sensors and regulators of intracellular pH," Nature Reviews Molecular Cell Biology, vol. 11, no. 1, pp. 50-61, jan 2010.

[150] M. Groll and R. Huber, "Purification, Crystallization, and Xâ̆̆̌̌Ray Analysis of the Yeast 20S Proteasome," in Methods in Enzymology, 2005, vol. 398, pp. 329-336.

[151] E. M. Huber, W. Heinemeyer, X. Li, C. S. Arendt, M. Hochstrasser, and M. Groll, "A unified mechanism for proteolysis and autocatalytic activation in the 20S proteasome." Nature communications, vol. 7, p. 10900, jan 2016.

[152] M. Groll, C. R. Berkers, H. L. Ploegh, and H. Ovaa, "Crystal Structure of the Boronic Acid-Based Proteasome Inhibitor Bortezomib in Complex with the Yeast 20S Proteasome," Structure, vol. 14, no. 3, pp. 451-456, mar 2006.

[153] M. Groll, R. Huber, and B. C. M. Potts, "Crystal Structures of Salinosporamide A (NPI-0052) and B (NPI-0047) in Complex with the 20S Proteasome Reveal Important Consequences of $\beta$-Lactone Ring Opening and a Mechanism for Irreversible Binding," 2006.

[154] J. E. Baldwin, R. C. Thomas, L. I. Kruse, and L. Silberman, "Rules for ring closure: ring formation by conjugate addition of oxygen nucleophiles," The Journal of Organic Chemistry, vol. 42, no. 24, pp. 3846-3852, nov 1977. 
[155] K. Gilmore, R. K. Mohamed, and I. V. Alabugin, "The Baldwin rules: revised and extended," Wiley Interdisciplinary Reviews: Computational Molecular Science, vol. 6, no. 5, pp. 487-514, sep 2016.

[156] I. Vilotijevic and T. F. Jamison, "Synthesis of marine polycyclic polyethers via endo-selective epoxide-opening cascades." Marine drugs, vol. 8, no. 3, pp. 763-809, mar 2010 .

[157] M. Arciniega, P. Beck, O. F. Lange, M. Groll, and R. Huber, "Differential global structural changes in the core particle of yeast and mouse proteasome induced by ligand binding." Proceedings of the National Academy of Sciences of the United States of America, vol. 111, no. 26, pp. 9479-84, jul 2014.

[158] P. Sledz, P. Unverdorben, F. Beck, G. Pfeifer, A. Schweitzer, F. Forster, and W. Baumeister, "Structure of the $26 \mathrm{~S}$ proteasome with ATP- S bound provides insights into the mechanism of nucleotide-dependent substrate translocation," Proceedings of the National Academy of Sciences, pp. 7264-7269, apr 2013.

[159] S. Asano, Y. Fukuda, F. Beck, A. Aufderheide, F. Forster, R. Danev, and W. Baumeister, "A molecular census of $26 \mathrm{~S}$ proteasomes in intact neurons," Science, vol. 347, no. 6220, pp. 439-442, jan 2015.

[160] H.-C. S. Yen, C. Espiritu, and E. C. Chang, "Rpn5 Is a Conserved Proteasome Subunit and Required for Proper Proteasome Localization and Assembly," Journal of Biological Chemistry, vol. 278, no. 33, pp. 30669-30676, aug 2003. 


\section{Acknowledgements}

I would like to thank Holger for giving me the opportunity to carry out my PhD Thesis in his department. I am very grateful to Holger and to my direct supervisor Ashwin, for their guidance and enthusiasm through the projects.

I also want to thank our collaboration partners Thomas and Gleb from the EMBL in Hamburg for their support and Kai and Ricardo from the University of Göttingen for their contribution to our work. The $20 \mathrm{~S}$ proteasome project would not have been possible without these excellent collaborations.

The Stark lab has been a great place to work over the past four years, and I would like to thank the current and former members of the lab. David, Erik, Steffi, Wen-Ti, Michael, Andrius, Juliane, Niels, Florian, Boris, Jan, Mario, Tobias, Sabrina, Georg, Felix, Lukas, Karl, Kashish, Fabian, Elham, Prakash, Frank, Dietmar, Gudrun, Dirk and Uwe. You all have contributed immensely to my personal and professional development during my $\mathrm{PhD}$.

I would also like to thank Ralf and Karin, for serving as my committee members and for their helpful comments.

This thesis would not have been possible without the moral support of many friends and my family. I would like to thank Tobi, Nils and my mother for all the love and encouragement. Thank you. 


\title{
Curriculum Vitae
}

\author{
Name: $\quad$ Jil Schrader \\ Date of birth: 27.Juli.1988 \\ Place of birth: Geseke, Germany
}

\section{Scientific education}

04/2013 - present PhD student in the Department of Structural Dynamics, Max Planck Institute for biophysical Chemistry, Göttingen, Thesis title: Structural Characterization of Proteasome Inhibition

04/2012 - 10/2012 Master Thesis in the Department of Structural Dynamics, Max Planck Institute for biophysical Chemistry, Göttingen, Thesis title: Mechanistic and Structural Analysis of Key Functional States of the AAA+ATPase p97

10/2010 - 10/2012 Georg-August University Göttingen: Studies in Microbiology and Biochemistry (MSc.), Specialization in Biochemistry and Structural biology

02/2010 - 06/2010 Bachelor Thesis in the Department of Molecular Structural Biology, Georg-August University, Göttingen, Thesis title: Expression und Reinigung der Spleißfaktoren Ntr1 und Ntr2

10/2007 - 08/2010 Georg-August University Göttingen: Studies in Biology (BSc.), Specialization in Molecular Biosciences

Göttingen, 2017 
